\title{
MATERNAL PERCEPTION OF FETAL MOVEMENTS: A QUALITATIVE DESCRIPTION
}

BY

\section{BILLIE BRADFORD}

\author{
A thesis \\ submitted to the Victoria University of Wellington \\ in fulfilment of the requirements for the degree of \\ Master of Midwifery
}

Victoria University of Wellington

2014 


\section{Abstract}

Background: Maternal perception of decreased fetal movements is a specific indicator of fetal compromise, notably in the context of poor fetal growth. There is currently no agreed numerical definition of decreased fetal movements, with subjective perception of a decrease on the part of the mother being the most significant definition clinically. Both qualitative and quantitative aspects of fetal activity may be important in identifying the compromised fetus. Yet, how pregnant women perceive and describe fetal activity is under-investigated by qualitative means. The aim of this study was to explore normal fetal activity, through firsthand descriptive accounts by pregnant women.

Methods: Using qualitative descriptive methodology, interviews were conducted with 19 low-risk women experiencing their first pregnancy, at two timepoints in their third trimester. Interview transcripts were later analysed using qualitative content analysis and patterns of fetal activity identified were then considered along-side the characteristics of the women and their birth outcomes.

Results: Fetal activity as described by pregnant women demonstrated a sustained increase in strength, frequency and variation from quickening until 28-32 weeks. Strength of movements continued to increase at term, but variation in movement types reduced. Kicking and jolting movements decreased at term with pushing or stretching movements dominating. In this study increased strength and frequency of movements at term as determined qualitatively by the mother was associated with higher mean customised birthweight of newborns, whilst decreased frequency was in all cases associated with either maternal obesity, customised birthweight under the $20^{\text {th }}$ centile or operative delivery for non-reassuring fetal status.

A novel finding of a complex pattern of fetal movements in relation to mealtimes is reported here. More than a third of participants (37\%) described marked increases in fetal movements in response to hunger, in many cases subsiding postprandially. The women who described this pattern in response to hunger and eating, subsequently gave birth to infants significantly smaller (mean difference 364gm) than those who did not describe a fetal response to hunger. Patterns of fetal movements were reported to be influenced by time of day, maternal position and 
activity, with an inverse relationship between maternal activity and fetal activity described by all participants.

Conclusions: Maternal descriptions of fetal movements with advancing gestation and in relation to time of day are consistent with fetal activity as described in ultrasound studies, lending reliability to the maternal account. Maternal perception of fetal movements in response to environmental stimuli such as maternal meals and maternal position-changes may constitute a maternal-fetal communication in the interests of maintaining the pregnancy. Pregnant women are reassured by fetal movements occurring at a usual time or situation, suggesting that maternal monitoring of fetal movements is a dynamic process taking into account a broad range of fetal functions.

Key Words: fetal movements, stillbirth, antenatal care, fetal development.

Billie Bradford has asserted her right under the Copyright Act 1994 to be identified as the author of this work. 


\section{Acknowledgements}

I would like to thank my supervisor Robyn Maude for her encouragement, support, and shared passion for fetal physiology. I would especially like to thank my friend Robin Cronin for her interest in the thesis and for providing moral support during difficult times.

I would also like to acknowledge the women who generously shared their pregnancies with me as participants in the research and the midwives who enthusiastically recruited women in their care to the study.

Thanks go to my mother Vanessa for instilling early in me a passion for science, research, wondering and discovery. Also to my father Alan and stepmother Rebecca for their enduring belief in me and ever-ready practical and loving support during the many trips to Wellington.

Last but not least, thanks to my husband Lee and children Iris, Roland, Gwen and William who have lost so much time with me to this work. Thank you for your patience and your pride in me. 


\section{Table of Contents}

\section{Contents}

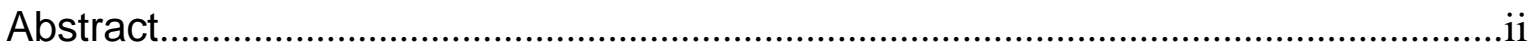

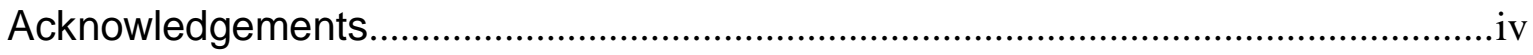

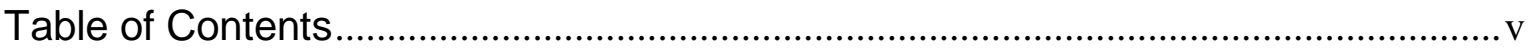

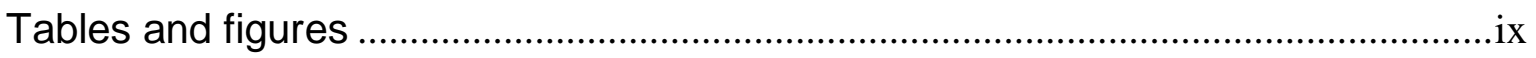

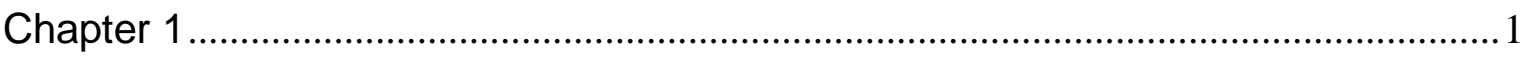

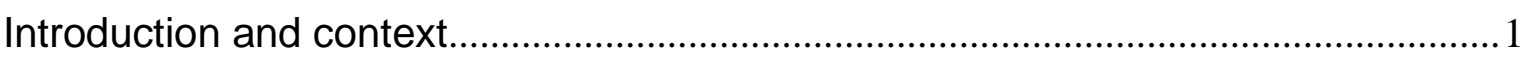

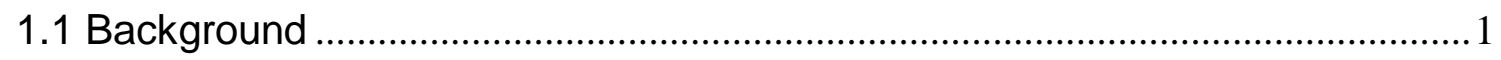

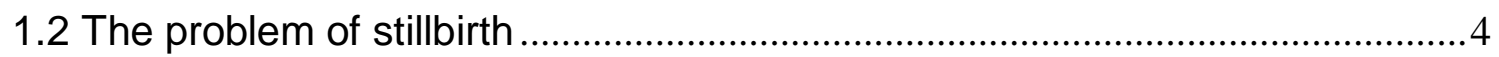

1.3 Maternal perception of fetal movements and fetal compromise ........................ 6

1.4 Clinical presentations with decreased fetal movements ....................................

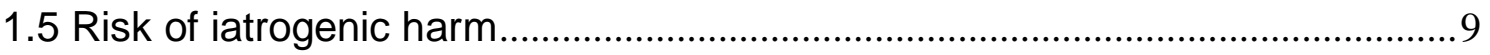

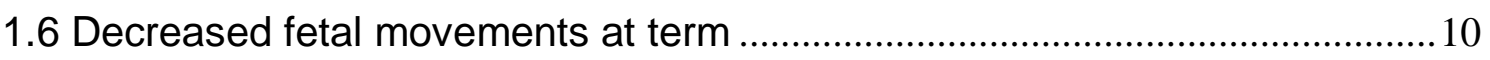

1.7 Midwifery imperative in antenatal surveillance ............................................... 10

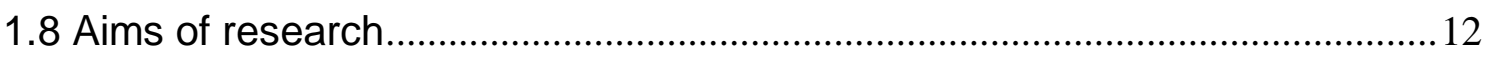

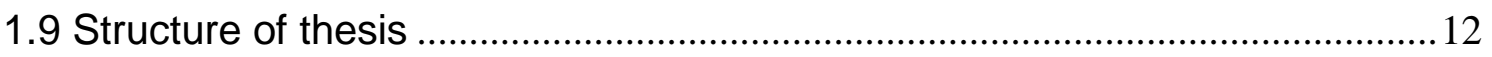

Chapter 2

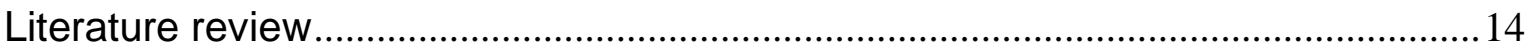

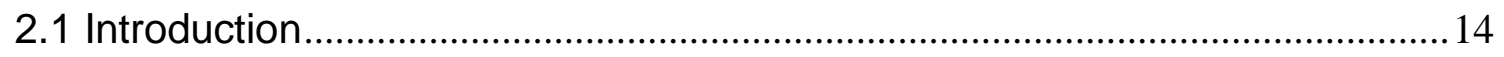

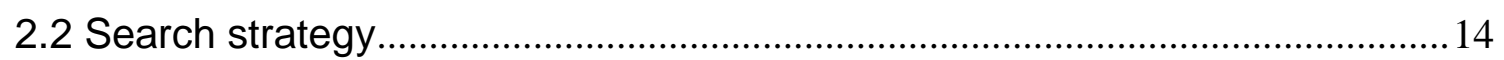

2.3 Section One: Ultrasound studies of fetal activity..............................................15

2.3.1 Fetal movements in early pregnancy ....................................................... 15

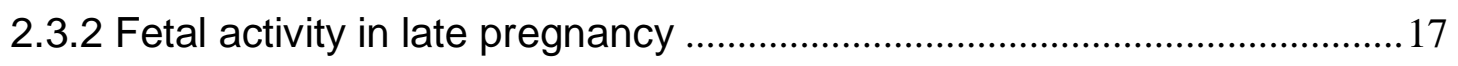

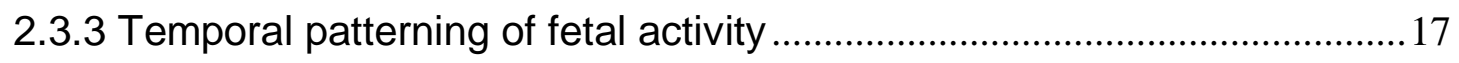

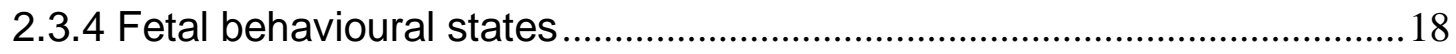

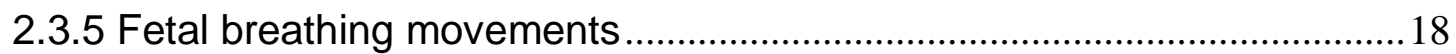

2.3.6 Ultrasound-observed fetal movements and pathology .............................19

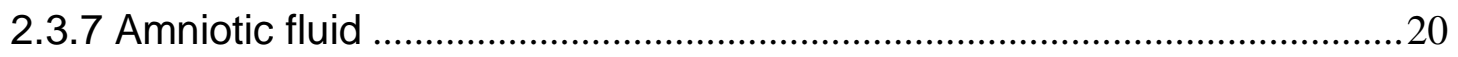

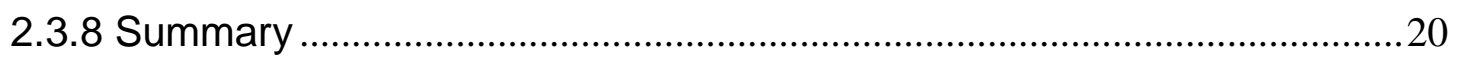

2.4 Section Two: Maternal perception of fetal movements ....................................20

2.4.1 Correlation between maternally-perceived fetal movements and objective detection ............................................................................................. 21

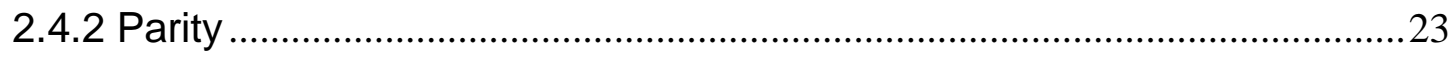

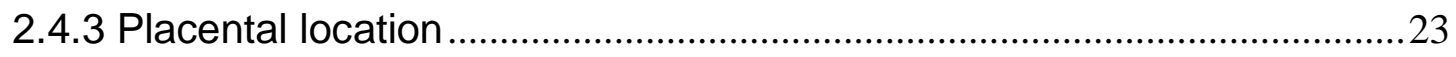




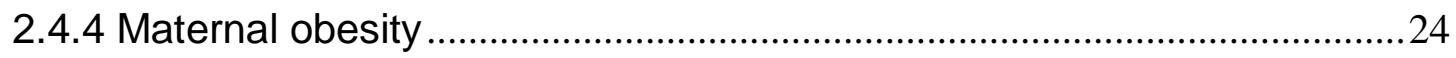

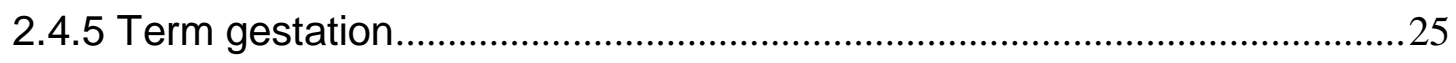

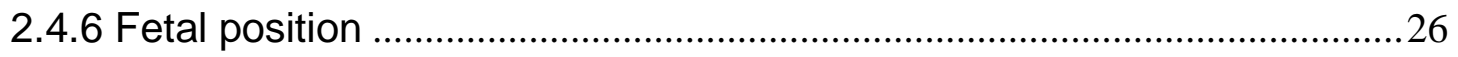

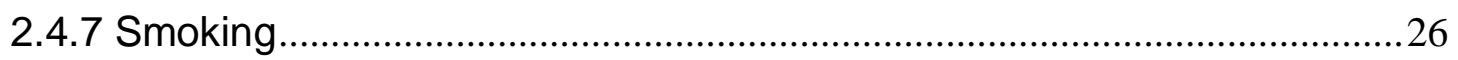

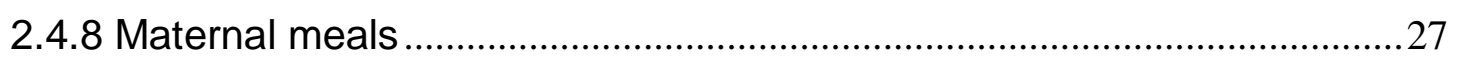

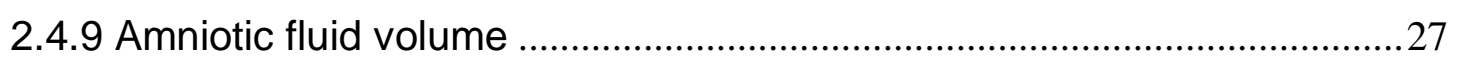

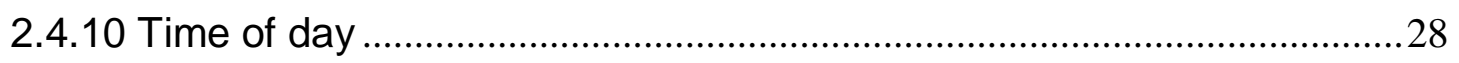

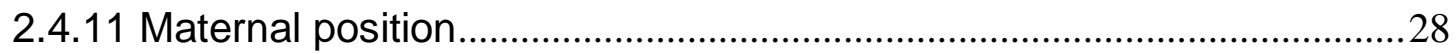

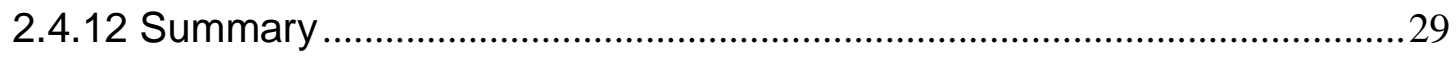

2.5 Section 3. Monitoring and assessing fetal movements....................................29

2.5.1 Establishing normal and pathological levels of fetal movement ..............29

2.5.2 Fetal movement monitoring methods ...........................................................

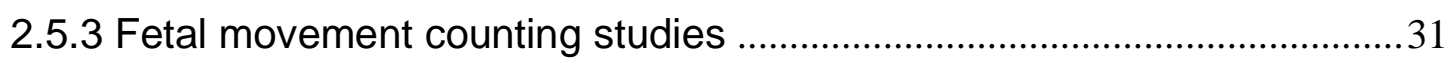

2.5.4 Acceptability of fetal movement monitoring methods: compliance and

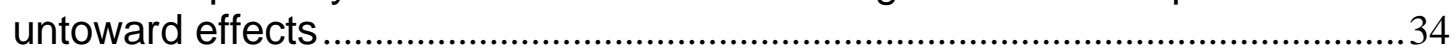

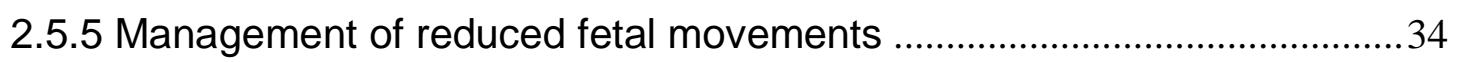

2.5.6 Practitioner views and current approaches to FM monitoring ...................37

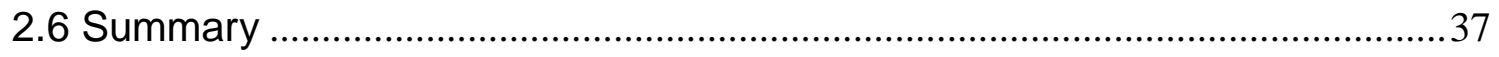

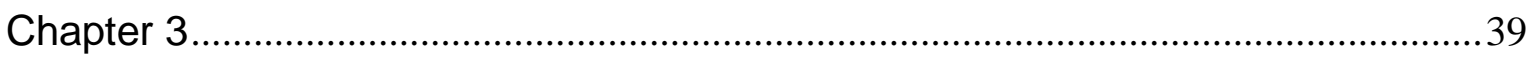

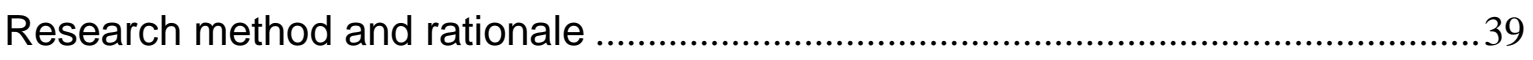

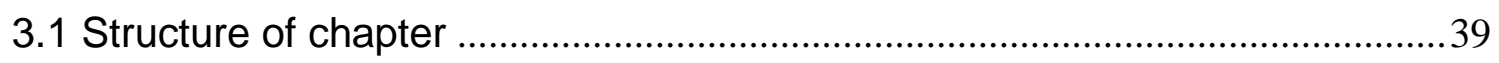

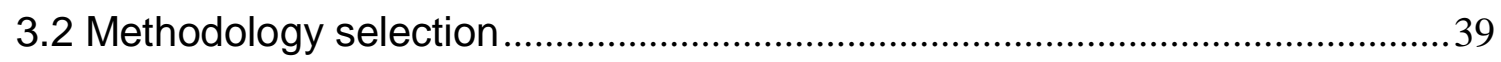

3.3 Maternal perception of fetal movements: A problem ripe for qualitative

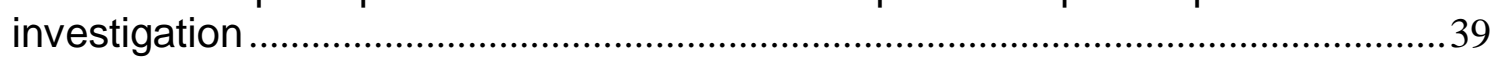

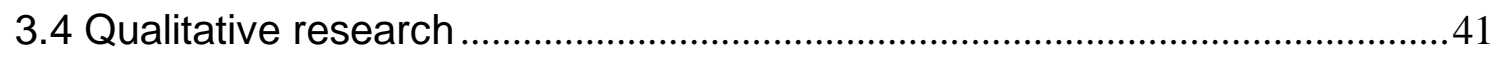

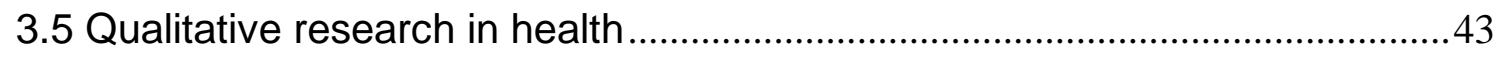

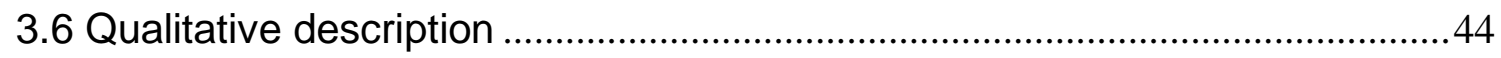

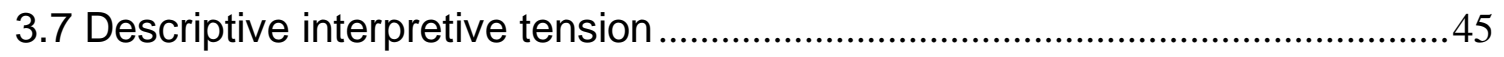

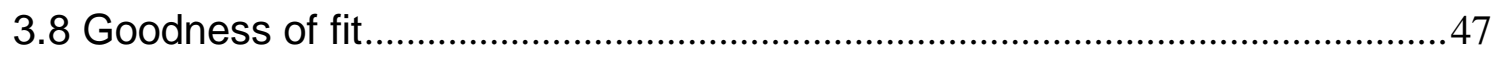

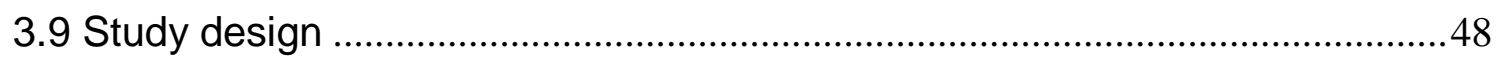

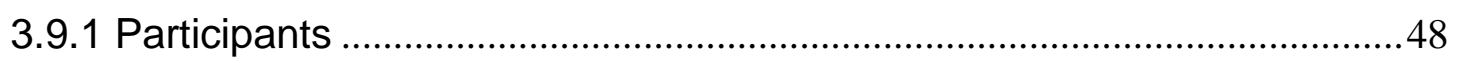

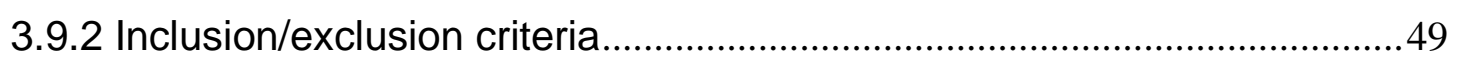

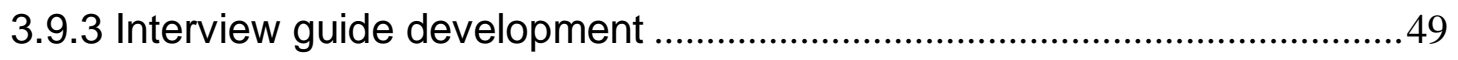

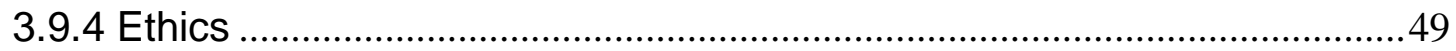

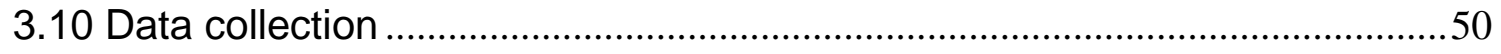

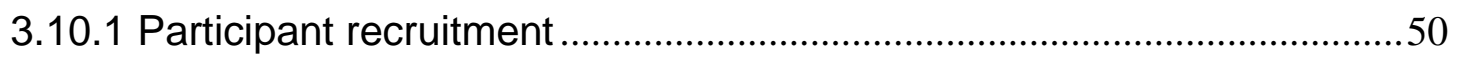

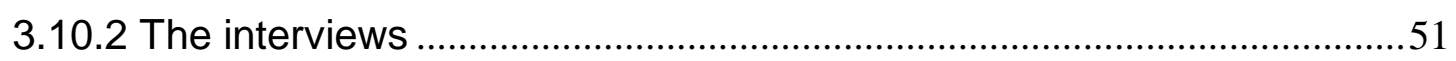




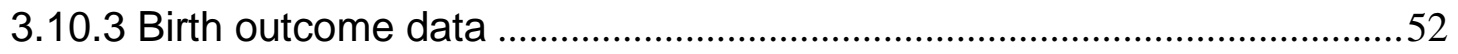

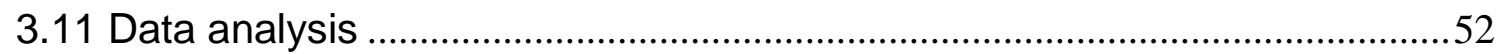

3.11.1 Qualitative content analysis of interview data ..........................................53

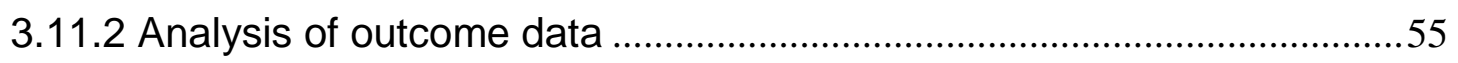

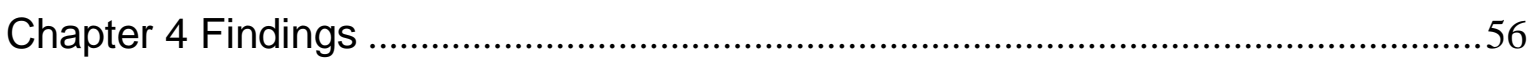

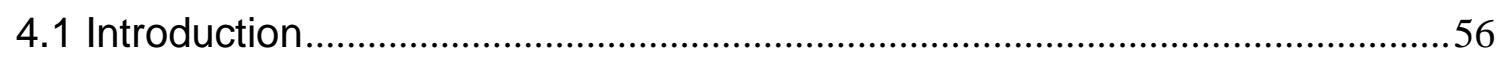

4.2 Section One: Maternal perception of fetal movements with advancing

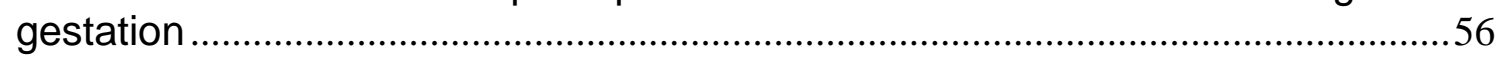

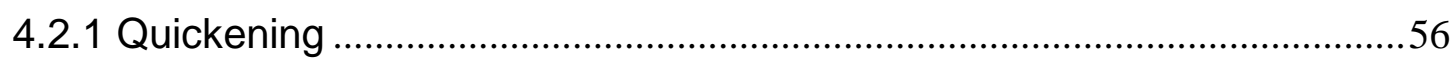

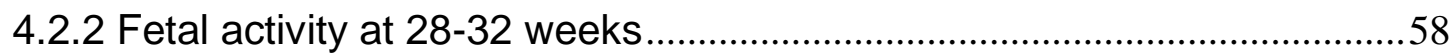

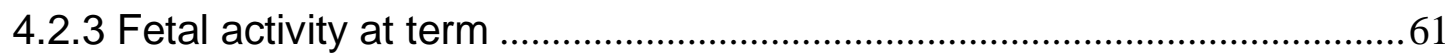

4.2.4 Variation in strength and frequency of fetal movements at term in

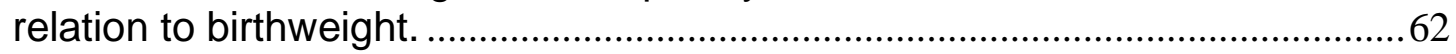

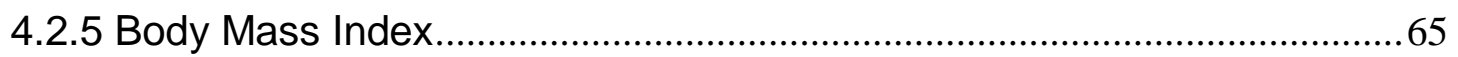

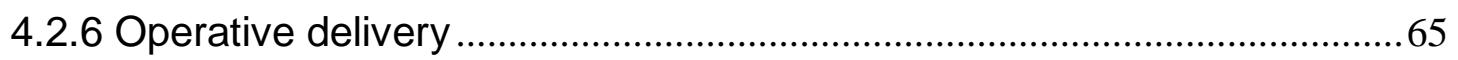

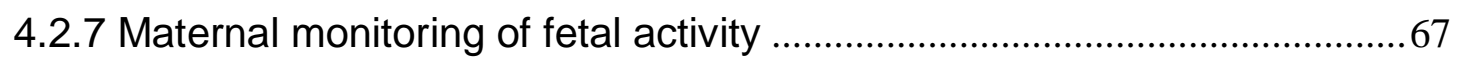

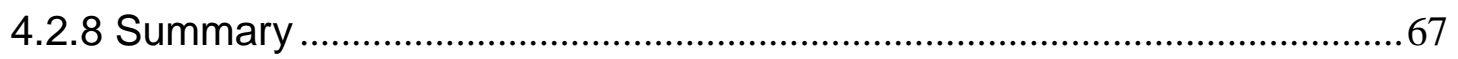

4.3 Section 2: Fetal response to maternal hunger and satiation ...........................68

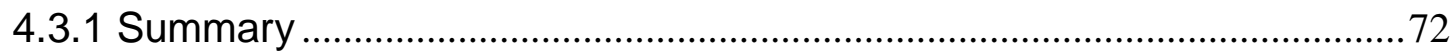

4.4 Section Three: Maternal perception of fetal movements: temporal, positional

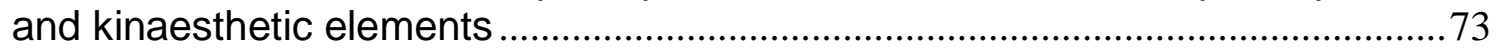

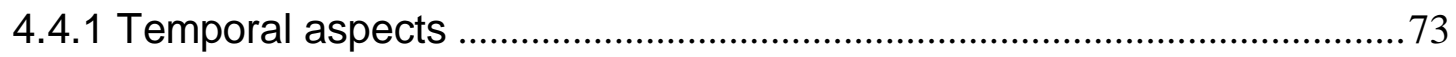

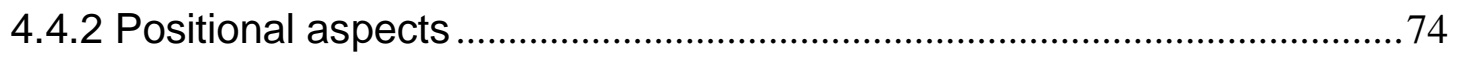

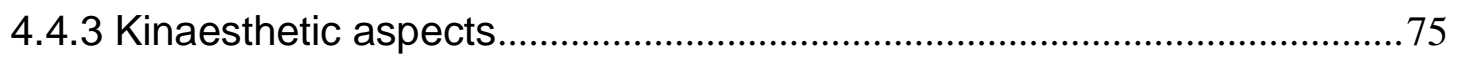

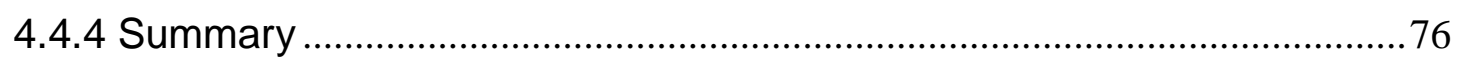

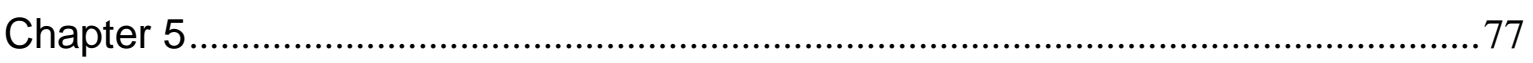

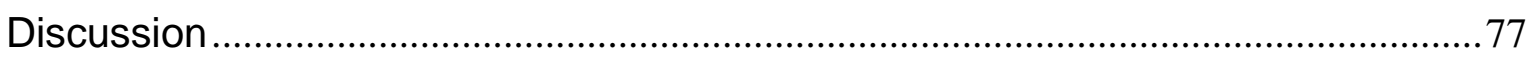

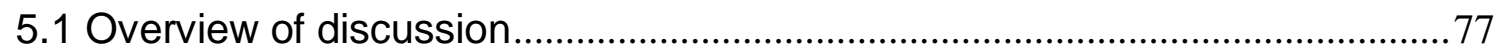

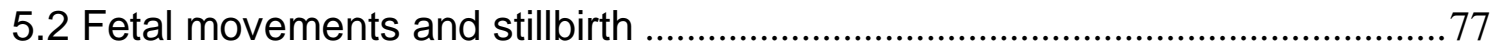

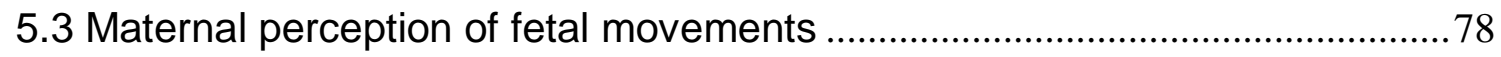

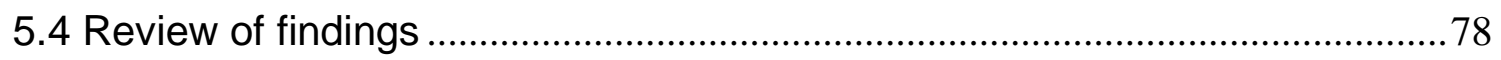

5.4.1 Fetal movements with advancing gestation - discussion .........................79

5.4.2 Fetal response to maternal hunger and satiation .....................................83

5.4.3 Temporal, positional and kinaesthetic elements ....................................90

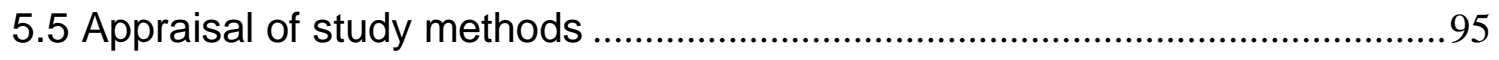

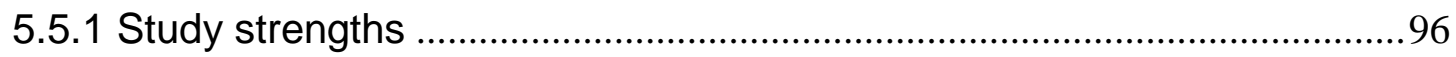

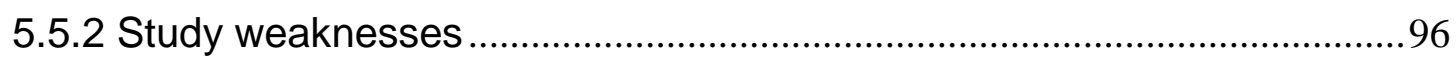

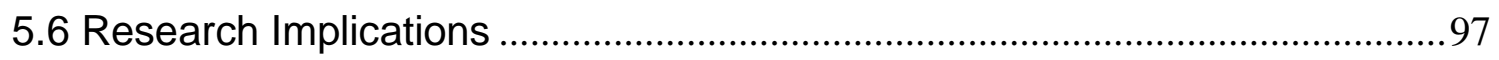




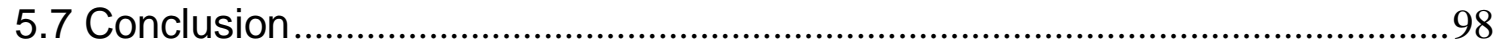

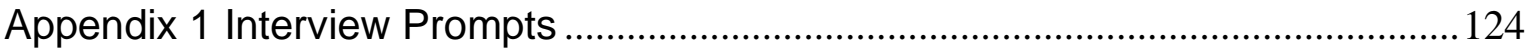

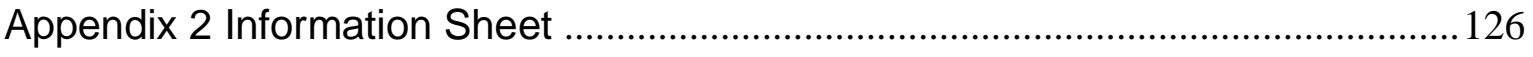

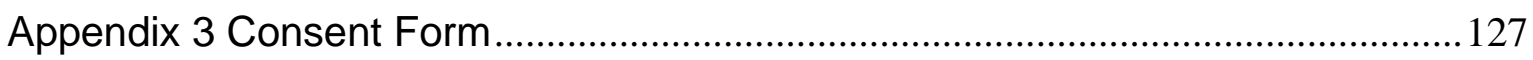

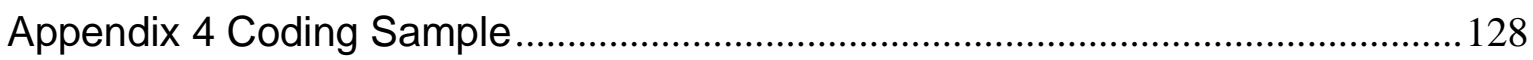




\section{Tables and figures}

\section{List of figures}

Figure 1 Staged responses to hunger and eating as described by participants...70

Figure 2 Maternal reports of increased fetal activity in the context of maternal hunger and subsequent birthweight

Figure 3 Maternal reports of increased fetal activity in the context of maternal hunger and subsequent birthweight expressed as a customised centile....

\section{List of tables}

Table 1 Distribution of changes in strength and frequency of fetal movements at term.

Table 2 Participant characteristics.

Table 3 Changes in strength and frequency of fetal movements in relation to customised birthweight. ...

Table 4 Participant characteristics and changes in strength and frequency of fetal movements at term. 


\section{Chapter 1}

\section{Introduction and context}

Quickening: to give or restore life to, to make alive, to vivify or revive, to animate (as the soul to the body). The Complete Oxford Dictionary p. 1491

\subsection{Background}

Maternal perception of normal fetal activity attests not only to the existence of the fetus, but to its viability and vitality. Prior to advances in laboratory pregnancy-testing and ultrasound scanning, the 'quickening' or first movements of the fetus felt by the mother gave further confirmation of pregnancy and estimation of due date (Gillieson, Dunlap, Nair, \& Pilon, 1985). In times past the baby in the womb was considered to have a soul only once its movements were felt by the mother (Duden, 1992). The term 'quick' has come to mean 'speedy' but its original meaning of lively or alive is retained in the words 'quicksilver', the 'quick' of the nail and 'quickening' of the human fetus.

Quickening and the progressive increase in strength, frequency and fluidity of fetal movements as pregnancy advances correlate with normal development and wellbeing of the fetus. Fetal movements are an expression of normal fetal anatomy, cardiovascular and neurological function (Heazell \& Frøen, 2008; Martin, 2008; Visser, Mulder, \& Tessa Ververs, 2010). Abnormal movements have been demonstrated in anencephalic fetuses and fetuses with structural abnormalities (Luchinger, Hadders-Algra, Van Kan, \& de Vries, 2008). The reduction or cessation of maternally perceived fetal movements has been described as an ominous sign by obstetricians since the $19^{\text {th }}$ century (Goodlin, 1979). In recent times maternal perception of reduced or absent fetal activity has been demonstrated to be a highly specific predictor of intra-uterine death (Heazell \& Frøen, 2008; Pearson \& Weaver, 1976; Sadovsky \& Yaffe, 1972).

Modern interest in decreased fetal movements as an alarm signal for fetal jeopardy began with two papers published in the 1970s. The first, a small case series of hospitalised women who kept daily fetal movement counts, demonstrated that fetal movements decreased in frequency over a one to three day period and 
then stopped for approximately one day prior to loss of fetal heart sounds (Sadovsky \& Yaffe, 1973). A subsequently published report confirmed the observation of diminished and then halted fetal activity prior to fetal death, proposing that a 'movement alarm' of 10 kicks per day counted by the pregnant woman could be used to monitor high risk pregnancies, with fewer than 10 movements being an indicator for early delivery (Pearson \& Weaver, 1976).

Early ultrasound studies confirmed that sluggish, infrequent or absent movements could be seen in compromised fetuses (Reinhold, 1973). At that time studies comparing maternally perceived fetal movements with those recorded by other means, such as ultrasound, demonstrated a high correlation between the two methods (Gettinger, Roberts, \& Campbell, 1978; Sadovsky, Mahler, Polishuk, \& Malkin, 1973), suggesting maternal monitoring of fetal activity had great potential as a tool for fetal surveillance. And so it was with considerable optimism that a boom in publications considering various methods of fetal movement monitoring followed (Froen, 2004).

In the subsequent 20 years, numerous studies were conducted evaluating the efficacy of more than a dozen different types of fetal movement counting methods to reduce perinatal mortality (Froen, 2004). Despite promising results from some early studies, later high-quality studies (Mangesi, Hofmeyr, \& Smith, 2007) show no formal fetal movement counting regime has proved successful at reducing perinatal deaths. Following the publication of a large randomised controlled trial (RCT) in The Lancet in 1989, which failed to demonstrate a benefit for formal fetal movement counting as part of routine antenatal care (Grant, Elbourne, Valentin, \& Alexander, 1989), interest in fetal movements as a method of antenatal screening dropped off. On the basis of the findings of this trial, evidence-based care guidelines have since been unable to recommend formal fetal movement monitoring as part of antenatal care for either low-risk or high-risk women (Mangesi et al., 2007). The National Institute for Health and Clinical Excellence (NICE) in the United Kingdom state in their guideline on antenatal care "routine formal fetal movement counting should not be offered" (NICE, 2008) p35.

The RCT by Grant and colleagues has since come under considerable criticism for the cluster randomisation procedure used, which meant control women often came into contact with women who were counting and, further, in many cases were themselves advised to count, suggesting contamination (Froen, 
2004). Also, lower stillbirth rates were reported during the period of the study than prior, which the authors suggested may have been attributed to the Hawthorne effect $^{1}$ (Grant et al., 1989). Despite the lack of evidence for formal fetal movement counting, reviews of the available literature suggest that there is likely to be benefit in monitoring fetal activity (Froen, 2004; Mangesi et al., 2007), although what form such monitoring should take is yet to be determined. The problem list for fetal movement monitoring to prevent stillbirth is long. Screening is hampered by lack of a clinically proven definition of normal fetal activity. In this void, myths and misinformation abound and health professionals' knowledge and understanding of fetal activity is often inconsistent (Heazell, Green, Wright, Flenady, \& Frøen, 2008). Women commonly present with concerns about fetal movements too late (Tveit et al., 2009; Unterscheider \& O'Donoghue, 2012), in many cases having been inappropriately dissuaded from seeking assessment by family members or by health professionals (Rådestad, 2010). Finally, an appropriate method for assessing the woman who presents with decreased fetal movements is yet to be determined (Hofmeyr \& Novikova, 2012). Considerable resources are expended in the evaluation of pregnancies with a complaint of decreased fetal movements. Yet women continue to experience stillbirths following apparently normal assessment for decreased fetal movements.

There is little doubt that maternal perception of decreased fetal movements is important as an indicator of fetal compromise, yet how this should be screened for, assessed and managed has not been determined. In the last decade a shift has occurred from considering numerical fetal movement alarms to acknowledging the importance of the woman's subjective perception (Preston et al., 2010; Tveit et al., 2009). Yet qualitative perception of fetal activity by women remains underinvestigated (Rådestad \& Lindgren, 2012; Raynes-Greenow, Gordon, Li, \& Hyett, 2013). Qualitative investigation of perception of fetal activity by pregnant women has the potential not only to inform understanding of the normal but to provide direction for further investigation of fetal movement pathology.

\footnotetext{
${ }^{1}$ The Hawthorne effect refers to a self-limiting change in subject's behaviour due to knowing that they are being observed as part of a study. Critical reviewers of the Grant trial have suggested that increased maternal awareness of fetal movements as a result of participating in the trial may have contributed to the reduction in late stillbirth during the study period when compared to prior, thereby negating the author's conclusions of no benefit for fetal movement monitoring.
} 


\subsection{The problem of stillbirth}

To any person who has delivered a stillborn child or been closely involved with a family facing the intense and often incapacitating grief that accompanies such a death, it is obvious that these events are to be avoided. The impact on women and families of a perinatal death is often devastating. In developed countries the usual expectation is that pregnancy will end with a live birth. Yet, one in 200 pregnancies after 22 weeks gestation ends in death of the fetus or newborn (Flenady, Middleton, et al., 2011). The loss of a baby before birth is a tragedy for all concerned, not only the parents of that child but also the grandparents, siblings, extended family and the wider community (Frøen et al., 2011; Goldenberg et al., 2011). Drive to avoid fetal death is behind many antenatal screening tests and high rates of interventions in labour such as continuous cardiotocograph (CTG) and emergency cesarean section (Goldenberg et al., 2011). Despite reductions in peripartum and infant death rates in recent decades, stillbirth rates in high-income countries have proved immutable to change.

There is little tolerance in our communities for such high rates of mortality from other causes. The stillbirth rate in New Zealand for example is four times the road death toll. Internationally the rate of stillbirth is 10 times that of the cot death rate (Lawn et al., 2011). Yet, prevention of stillbirths does not command the same level of public awareness and research as other mortality. This may partly be due to the commonly held view that babies who die in the womb are usually malformed or somehow 'not meant to be', an outlook that has been termed "inappropriate fatalism" (Goldenberg et al., 2011, p. 1798). Congenital abnormality is a significant cause of stillbirth, but accounts for a minority of cases overall. In fact, half of all stillbirths in developed countries occur in normally-formed babies of more than 28 weeks gestation (Goldenberg et al., 2011). These are babies which, if born alive, would normally have been expected to survive.

Risk of stillbirth, and most especially stillbirth in the context of intra-uterine growth restriction (IUGR), increases as pregnancy advances. In New Zealand 5.3 babies per 1000 are stillborn, a rate that is comparable to that of other developed countries (PMMRC, 2013). One quarter of these stillbirths are unexplained, and half of these occur at term (more than 37 weeks gestation). Data from the United 
States indicate that rates of fetal death are lowest between 24 and 37 weeks of pregnancy, steadily increasing over the 37 to 43 week period (Silver, 2011).

A better understanding of possible causes of late stillbirth in otherwise normal fetuses, and improved tools for fetal assessment are urgently needed. A proportion of stillbirths are likely to be preventable and research into better means for assessing and monitoring the wellbeing of the fetus could translate into a very real reduction in mortality. Causes and prevention of stillbirth have not been made a research priority and a reduction in rates of stillbirth have stalled in developed countries (Bhutta et al., 2011; Goldenberg et al., 2011; McCowan, GeorgeHaddad, Stacey, \& Thompson, 2007). The reduction in rates of cot death has been helped by research into the cause and prevention. A concentration of research efforts into stillbirth prevention may bring similar gains for the many families affected.

Stillbirth rates vary between high income countries, suggesting that there is still potential for rates to be reduced. In high-income countries, stillbirth is associated with social disadvantage, maternal obesity, advanced maternal age and smoking (Flenady et al, 2011). Maternal obesity has a linear relationship with perinatal mortality (Salihu, 2008; Tomasina Stacey et al., 2011b) and rising levels of obesity in developed countries threaten to undermine gains made in perinatal care. Women in developed countries who live in areas of greater social deprivation bear the greater burden in rates of stillbirth (Flenady, Koopmans, et al., 2011). Levels of smoking and maternal obesity are higher amongst these populations and may be considered markers for socio-economic disadvantage (PMMRC, 2013). Reducing stillbirth rates is also important as part of a wider picture of maternal and child health. The same factors that increase risk of stillbirth also increase risk of neonatal and infant death, of preterm birth and newborn central nervous system injury (Salihu, 2008). Advances made in stillbirth prevention have the potential to reduce the incidence of neonatal morbidity and mortality from the same causes.

Audit of perinatal deaths provides opportunity to identify targets for improvement in care or services so that death rates might be reduced. A Norwegian audit of perinatal mortality revealed mismanagement of fetal growth restriction and/or decreased fetal movements are common failures in antenatal care (Saastad, Vangen, \& Frederik Frøen, 2007). In a subsequent study of women 
presenting with decreased fetal movements, $19 \%$ of pregnant women who had a stillbirth presented at hospital with a live fetus, in every case (18 cases) the fetus died within a week of the visit (Tveit, Saastad, Stray-Pedersen, Børdahl, \& Frøen, 2009).

In a recent Lancet series on stillbirth, a number of priorities were identified for research. Among the top 10 priorities were: "characterising the fetal response to an adverse intra-uterine environment to develop improved means of clinical assessment of fetal wellbeing" and determining "for women who report decreased fetal movements what is the optimum management to reduce the risk of stillbirth in term and preterm pregnancies" pp 103-104 (Flenady, Middleton, et al., 2011).

\subsection{Maternal perception of fetal movements and fetal compromise}

Fetal death is often heralded by maternal concerns about a reduction in fetal movements. The change may be short-term or may have occurred over a longer period (Malm, Lindgren, \& Rådestad, 2010). Maternal perception of a change in fetal movements is very often the first sign of fetal compromise. A reduction in gross body movements in response to diminished oxygen supply is known to occur in fetal lambs (Jackson, Forouzan, \& Cohen, 1991). In the human fetus, decreased movements are thought to be a compensatory measure, reducing output in the context of decreased supply of oxygen and nutrients (Hijazi, Callan, \& East, 2010; Warrander \& Heazell, 2011).

Formal fetal movement counting is the predominant type of fetal movement monitoring investigated to date, yet pregnant women's concerns about fetal movements in the context of fetal jeopardy are not always necessarily about a simple reduction in frequency of movements. In many cases women report vague concerns about fetal movements, stating only that 'something is not right' (Goodlin, 1979). Midwives have long acknowledged that a change in pattern of fetal movements may indicate a problem (Bennet \& Brown, 1999). Stacey and colleagues (2011) reported that women who had experienced a stillbirth were significantly more likely to indicate a reduction in strength and frequency of fetal movements in the weeks preceding the fetal loss than control women with ongoing 
pregnancies (Stacey et al., 2011a). This finding supports the notion that qualitative aspects of perceived fetal activity may be predictive of fetal compromise.

Neurological studies indicate that quality of movements provides better indication of fetal neurological status than quantity (Reissland \& Francis, 2010; Sival, Visser, \& Prechtl, 1992b). Quality of fetal movements is altered in pregnancies affected by gestational diabetes (Kainer, Prechtl, Engele, \& Eispeiler, 1997), intra-uterine growth restriction (Bekedam, Visser, de Vries, \& Prechtl, 1985), reduced liquor volume (Sival, Visser, \& Prechtl, 1990) and fetal neurological impairment (Morokuma et al., 2007).

In two Swedish studies investigating women's premonitions that their baby had died prior to confirmation of stillbirth, participants reported sensing that they had 'lost contact' with their baby, specifying that their baby didn't move according to their usual pattern or respond in situations that would normally have evoked movements (Erlandsson, Lindgren, Davidsson-Bremborg, \& Rådestad, 2012; Malm, Lindgren, \& Rådestad, 2010).

For most women it is important to feel their baby move every day (Frøen et al., 2008a). Women who note changes in their baby's movements will often report their concerns to a health-care provider, regardless of whether or not they have been advised to do so. Thus fetal movement screening goes on in the community as an unstructured activity initiated by women themselves (Frøen et al., 2008a). In an Australian cross-sectional survey of fetal movements maternal qualitative assessment of fetal activity was demonstrated to be of value to clinicians (RaynesGreenow et al., 2013). Yet there is a paucity of available research evidence to inform health professionals' evaluation of fetal movement quality.

\subsection{Clinical presentations with decreased fetal movements}

In approximately half of stillbirth cases the woman reports a history of decreased fetal movements prior to diagnosis (Efkarpidis, Alexopoulos, Kean, Liu, \& Fay, 2004). Women who present with decreased fetal movements are at higher risk of complications, including delivery of a growth-restricted baby, preterm birth, and both actual and impending fetal death (Dutton et al., 2012; Tveit et al., 2009; Olesen \& Svare, 2004). Rates of fetal growth restriction in particular are elevated 
in this group (Tveit et al., 2009; Tveit, Saastad, Stray-Pedersen, Børdahl, \& Frøen, 2010). Fetal growth restriction is a significant precursor to fetal death that is frequently underdiagnosed (Froen, Gardosi, Thurmann, Francis, \& StrayPedersen, 2004; McCowan et al., 2007). Evidence suggests that fetal death is reduced when small babies are identified sooner and able to be monitored (Lindqvist \& Molin, 2005). Improved identification of growth-restricted fetuses is a key benefit of assessment of pregnancies where there is a complaint of decreased fetal movements (Preston et al., 2010; Saastad, Winje, Stray Pedersen, \& Frøen, 2011; Sinha, Sharma, Nallaswamy, Jayagopal, \& Bhatti, 2007; Tveit et al., 2009).

Presentations with decreased fetal movements have also been shown to be associated with maternal smoking (Coppens, 2001; Habek, 2007; Tveit, Saastad, Stray-Pedersen, Børdahl, \& Frøen, 2010), maternal obesity (Tuffnell, Cartmill, \& Lilford, 1991; Tveit et al., 2009), placental pathology (Warrander \& Heazell, 2011; Warrander et al., 2012) and reduced liquor volume (Ahn, Phelan, Smith, Jacobs, \& Rutherford, 1987). All such factors are in turn associated with increased incidence of fetal death.

Worrisome though these associations may be, screening and assessment of fetal activity amongst pregnant women is not straightforward. Reduced fetal movements may also be caused by benign associations such as anterior placenta (Fisher, 1999b), normal sleep patterns in the term fetus, women who have been busy (Harrington et al., 1998), or are in an upright position (Minors \& Waterhouse, 1979). Between five and $15 \%$ of women will contact their health care provider with a concern about reduced fetal movements (Froen, 2004; Tveit et al., 2009). The majority of these cases will have a normal perinatal course (Fisher, 1999a; Harrington et al., 1998). Some studies suggest that as many as $50 \%$ of women may have concerns about fetal movements at some point in their pregnancy (Hijazi et al., 2010). Maternal perception of reduced fetal movements has clinical significance as a predictor of fetal compromise, but a relatively low positive predictive value of $2 \%$ to $7 \%$ for stillbirth (Unterscheider \& O'Donoghue, 2012). When assessing a complaint of reduced fetal movements, differentiating women with an 'at-risk fetus' from normal cases is difficult, but especially important. 


\subsection{Risk of iatrogenic harm}

The key intervention to prevent fetal death in cases of decreased fetal movements is delivery (Pearson \& Weaver, 1976). But all is not necessarily well for the neonate that is delivered early. The fetus continues to make significant developmental changes throughout gestation up to and including the last weeks of pregnancy, including laying down fat stores, producing surfactant, undergoing brain growth and moderating their behavioural states. Early delivery puts the neonate at risk of separation from the mother, difficulty establishing feeding, infection and jaundice requiring phototherapy, and also increases the risk of neurodevelopmental disability, and school and behaviour problems in the longer term (Samra, McGrath, \& Wehbe, 2011). Whilst it is true that such sequelae are more common and more severe at earlier gestations, even at gestations of 34 to 37 weeks, outcomes for those born early appear to be poorer than those born at term (Allen, 2002; Samra et al., 2011). The 34-37 week fetus experiences a critical period in brain growth. During the last six weeks of gestation $35 \%$ of the fetal brain and $47 \%$ of the cortical volume is acquired, whilst the myelinated white-matter increases five-fold (Samra et al., 2011). This rapid growth will occur postnatally in the infant born early, but ideally occurs in the quiescent environment of the womb where inputs and experiences are different from those outside the uterus (Allen, 2002). The decision to deliver early, even in term gestations, is a decision that should not be taken lightly.

When outcomes of infants born electively (that is without clinical indication for timing of delivery) at 37, 38 and 39 weeks are considered, earlier delivery is associated with increased respiratory problems, neonatal admissions, infection and mortality (Yee, Amin, \& Wood, 2008). Mounting evidence in favour of allowing the fetus to gestate until term has led to changes in clinical guidelines for elective cesarean section, whereby 39 weeks is the recommended timing as opposed to any time after 37 weeks (Royal Australian and New Zealand College of Obstetricians and Gynecologists, 2012; Yee et al., 2008). The art of antenatal care is to balance the needs of the fetus with those of the neonate. 


\subsection{Decreased fetal movements at term}

Differentiating normal from abnormal fetal activity is nowhere more problematic than in term pregnancies. There is a commonly held view that fetal movements normally cease prior to labour. In cases of delayed presentation with absent fetal movements and fetal death, the woman has very often been dissuaded from reporting her concerns about movements by the belief that movements normally reduce at term or prior to labour (Rådestad, 2010).

The nature of fetal movements change as pregnancy advances, but this change is poorly defined. Ultrasound studies of fetal activity show a progression from frequent jerky movements in early pregnancy to complex co-ordinated sequences of movements near term (D'Elia, Pighetti, Moccia, \& Santangelo, 2001; Pillai, James, \& Parker, 1992). Accordingly, pregnant women describe short, sharp punchy movements giving way to smooth sustained churning or rolling sensations (Raynes-Greenow et al., 2013). As gestation advances in a normal pregnancy the fetus has longer sleep periods, more deliberate movements and fewer 'spastic type' movements (D'Elia et al., 2001; Pillai \& James, 1990). This developmental change in pattern and the concomitant change in perception of fetal actions by the mother may explain why fetal movements have at times been described as becoming less frequent closer to term (Rådestad \& Lindgren, 2012). In the Auckland Stillbirth study a decrease in fetal movements was associated with stillbirth in women who were less than 37 weeks, but this association was not statistically significant in term women (Stacey et al., 2011a). Exploration of the nature of the change in fetal activity at term as perceived by the woman is required so that the normally-developing term fetus can be spared iatrogenic harm from early birth, and the compromised fetus can be appropriately identified.

\subsection{Midwifery imperative in antenatal surveillance}

Enquiring after the movements of the fetus is a routine part of modern antenatal care (Flenady et al., 2009; Heazell, Green, Wright, Flenady, \& Frøen, 2008). Pregnant women often seek information about fetal movements, reporting any concerns about fetal activity and wanting to know if their progress is normal. 
For women, pregnancy can be a time of worry (Bondas \& Eriksson, 2001). Although stillbirth remains a taboo subject, concerns about normal progress on behalf of the woman are often present and may be a normal part of growing attachment to her baby.

The role of the midwife is to work in partnership with the woman, providing care and information, supporting the woman to be self-determining and to realise her aspirations for her birth, her baby and her entry to motherhood (New Zealand College of Midwives, 2008). Working in partnership with the woman involves a bidirectional sharing of information about the pregnancy. The midwife provides information to the woman to better enable her to monitor her baby's wellbeing, including advice as to when to seek additional assessment, such as for vaginal bleeding, preterm labour or concerning changes in fetal activity (New Zealand College of Midwives, 2008). The woman in turn shares information about her own family and healthcare history and also about her impressions of her growing and active fetus (Baston, 2003). A healthy exchange provides the midwife with information about fetal wellbeing via the woman's observations, and the woman with information about when to seek further assessment.

Pregnancy and childbirth are for the most part healthy normal life events that require little intervention. For this reason, the potential to cause iatrogenic harm by intervening unnecessarily is keenly felt by maternity care providers. The midwifery imperative is to support normal physiological birth, using any intervention sparingly and with full regard for potential harm. Reports of decreased movements are vastly more common amongst pregnant women than stillbirth. Screening for normal fetal activity involves balancing the risks of iatrogenic harm from unwarranted intervention (such as induced early birth) and failure by omission where nothing is done for a fetus in jeopardy.

Many women wait more than 48 hours with reduced or absent fetal movements before seeking assessment (Tveit et al., 2009). Clinical practice guidelines encourage giving women information about fetal movements so that such late presentations may be avoided (Preston et al., 2010). However, the nature of normal fetal activity as perceived by pregnant women is under-explored making the task of giving women information difficult. Fetal movement counting can cause anxiety in some women, and the effects on pregnant women of 
promoting monitoring of fetal movements by broad definitions have not been evaluated. Lack of a useful definition of normal fetal activity, proven screening methods and quality evidence to guide appropriate assessment of complaints leaves the midwife currently with little reliable guidance in dealing with this clinical problem.

Midwifery is based on partnership with women. Pregnant women are the most vested keepers of their children. In order to advance understandings of fetal activity as an indicator of fetal wellbeing, new approaches must be considered. For this study, a return to the source, the pregnant woman, was deemed necessary.

\subsection{Aims of research}

The broad intention of this research was to provide a qualitative description of normal fetal activity as perceived by the pregnant woman. It is hoped such an account might:

a) assist midwives and obstetricians in providing information to women about normal fetal movements

b) provide clues as to where further research efforts might be directed in order to better understand maternal perception of decreased fetal movements and early warning of fetal compromise.

\subsection{Structure of thesis}

This thesis has begun with an overview of maternal perception of decreased fetal movements and stillbirth to provide a context for the clinical problem of reduced fetal movements and stillbirth, so that the impetus for this study and rationale for the methods selected can be understood. Chapter Two constitutes a review of the available literature on fetal movements, including present scientific understandings of human fetal activity based largely on ultrasound studies, known factors influencing perception of fetal movements by the pregnant woman, and methods for assessment of complaints of reduced fetal movements. In Chapter Three the methods and rationale for their use in the context of the problem being investigated is presented. 
The body of the thesis follows and is presented as a single-findings chapter with three sections devoted to the three main findings. Section One provides a chronological description of fetal movement types over the course of pregnancy and is entitled Maternal Perception of Fetal Movements with Advancing Gestation'. The subject of normal fetal movement perception at term is problematic and this study adds a maternal account of movements with advancing gestation.

In Section Two, a novel and unexpected finding - 'Fetal Reponses to Maternal Hunger and Satiation - is presented. Staged fetal responses to maternal hunger and satiation were provided by over a third of participants. These women were later found to deliver smaller babies. Aberrant fetal growth is a significant precursor to stillbirth and any relationship between fetal movements and growth warrants notice.

Section Three is entitled 'Maternal Perception of Fetal Movements temporal, positional and kinaesthetic elements'. Maternal concerns about fetal activity prior to diagnosis of stillbirth often relate to a change in pattern. Patterns of fetal activity, as described by participants in the study, and the influence of time-ofday, maternal position, maternal activity and rest, are presented in this section.

Chapter Four, a discussion chapter, concludes the thesis by firstly revisiting the impetus for the study, aims and rationale for the approach used. Discussion of the key findings is then provided in three sections, including the advancement of hypothetical connections between the collected descriptions and the problem of reduced fetal movements and stillbirth. Consideration is then given to the strengths and limitations of the study with concluding thoughts for future research in this area. 


\section{Chapter 2}

\section{Literature review}

\subsection{Introduction}

This literature review is concerned with published literature on the motor activity of the human fetus, maternal perception of fetal activity, and screening and management of fetal movements during pregnancy. The review is divided into three sections.

Section One reviews primarily ultrasound studies of motor development of the fetus over the course of pregnancy, including: behavioural-state development, temporal patterning, and influence of pathological conditions on fetal movements. This section provides a summary of present scientific knowledge of fetal movements on which clinical understandings may be based.

Section Two considers literature in relation to the perception of fetal movements by the pregnant woman; correlation between maternally-perceived movements and ultrasound; and factors thought to influence maternal perception of fetal movements such as parity, placental location, stage of gestation, fetal position and level of amniotic fluid; together with maternal obesity, smoking, and meals. This section summarises present knowledge of maternal perception of fetal movements, highlighting the variation in the pregnant population and numerous gaps in understanding.

Section Three comprises a review of methods of screening and monitoring fetal movements, including attempts to establish fetal movement count limits, methods of formal fetal movement monitoring, their acceptability to women, assessment of pregnancy when fetal movements are reduced, and practitioner views on fetal movement assessment. Problems are highlighted and opportunities for further study are identified.

\subsection{Search strategy}


Literature sources were identified by searching Medline and Cinahl using the term 'fetal movement(s)'. Literature searches commenced in 2010 and were repeated at three-month intervals until January 2014. Sources included academic papers published in English after 1970, key midwifery and obstetric textbooks and $\mathrm{PhD}$ theses on fetal movements. A secondary search strategy involved further identification of relevant publications via reference lists of those sources identified in the initial search and searching by prominent authors and author groups. This secondary hand-search identified numerous older works of interest. The subject of fetal movements enjoyed considerable interest in the 1970 s and $80 \mathrm{~s}$, with a significant amount of research being published over that time. A drop-off in interest from the early 1990s until recent times meant that some valuable lines of inquiry have remained undeveloped since the 80 s. Seminal works from that period remain the last word on parts of the subject and so have been included in this review.

\subsection{Section One: Ultrasound studies of fetal activity}

Information about fetal activity may be derived from a range of sources, including by palpation of the abdomen, pressure-measuring devices such as strain gauges and piezoelectric crystals, maternal perception and ultrasound evaluation. Prior to the advent of ultrasound, observation of fetal activity for the purposes of study was limited to aborted fetuses, whose survival outside the uterus was limited to minutes, and to exteriorised fetal animals (Marsal, 1983). Study of fetal movements, their normal developmental course and changes in the context of pathology is useful in understanding the origins of human behaviour and also in advancing knowledge of perinatal pathology.

\subsubsection{Fetal movements in early pregnancy}

The earliest ultrasound studies of fetal movements were made by Reinhold (1973), who observed spontaneous fetal movements as early as eight weeks gestation, describing 'lively' (whole body) and 'inert' (isolated limb) movements, and elicited movements, where the fetus moved in response to the examiner 
palpating the uterus through the mother's abdominal wall as if to waken it (Reinhold, 1973).

By 1978 Birnholz and colleagues had identified 11 different spontaneous movement patterns, including the elicited movement response noted by Reinhold (Birnholz, Stephens, \& Faria, 1978). Later a comprehensive catalogue of fetal movement types seen on ultrasound, the gestation at which they first occur and their incidence according to gestation was provided by de Vries and colleagues (de Vries, Visser, \& Prechtl, 1982, 1985). These early classifications remain the basis of knowledge of fetal motor development and behaviour with some ongoing refinement as sonographic technology advances (Andonotopo, Stanojevic, Kurjak, Azumendi, \& Carrera, 2004).

Early investigators noted that fetal movement types observed by ultrasound were very much like those seen in the newborn (e.g. stretches and startles) and so gave them the same names (de Vries et al., 1985; Visser et al., 2010). Studies of fetal activity in the first half of pregnancy show a clear developmental trend. First movements are simple and involve the whole body. With advancing gestation, movements increase in frequency and complexity, with breathing, sucking, swallowing, startles, hiccups, isolated limb movements, stretches and yawns being added to the repertoire (de Vries et al., 1982, 1985; Marsal, 1983).

Longitudinal studies reveal that incidences of many movement types change as pregnancy advances. Some movements increase in frequency with progressive maturity of the fetus, such as fetal breathing (Birnholz et al., 1978; D’Elia et al., 2001; Groome, Swiber, Holland, Bentz, \& Atterbury, 1998), eye movements (Birnholz et al., 1978; Roodenburg, Wladirimoff, van Es, \& Prechtl, 1991) jaw movements (Roodenburg et al., 1991), and sucking and swallowing (de Vries et al., 1985; Roodenburg et al., 1991). Others, such as stretches and startles, first appear at 10 weeks and nine weeks respectively, and increase in frequency as gestation advances until they reach a plateau, after which they decrease ( de Vries et al., 1985; Roodenburg et al., 1991). Periods of quiescence increase in length as gestation advances and the fetus displays fewer jerky or spastic movements. Hiccups dominate the fetal movement pattern in early pregnancy, comprising $20 \%$ of total fetal movements according to de Vries and colleagues (1985). Fetal movements generally become more organised as the fetus matures (Birnholz et al., 1978; D’Elia et al., 2001; Groome et al., 1998). 
Increasing organisation of fetal movements throughout pregnancy is thought to be indicative of normal neurological development, becoming even more pronounced in late pregnancy.

\subsubsection{Fetal activity in late pregnancy}

The hallmark of fetal activity in later pregnancy is a progressive change from chaotic to organized fetal movement patterns (D'Elia et al., 2001; Pillai et al., 1992). Stretches, startles and jerky movements continue to decline in frequency as pregnancy advances. Fetal breathing movements, providing practice for postnatal breathing, occur more frequently at later gestations and coincide with periods of quiescence (D'Elia et al., 2001; Pillai et al., 1992). Periods of quiescence continue to increase in length and are positively correlated with mouthing movements. Gross body movements on the other hand are positively correlated with eye movements and 'other mouth movements' such as opening and closing the mouth, protruding the tongue and swallowing (D'Elia et al., 2001; Pillai et al., 1992). This clustering of movement types is thought to be indicative of normal neurological development, preparing the fetus for those important tasks of neonatal life: co-ordination of breathing, sucking and swallowing. By 36 weeks these spells of activity and quiescence develop an episodic character.

\subsubsection{Temporal patterning of fetal activity}

Fetal activity has a rich temporal pattern. Behavioural-state cycling seen in the term fetus persists for the first four months of life after birth and has also been documented in other mammals (Robertson \& Dierker, 2003). Cycling through periods of activity and rest occurs not only through short time-periods (minutes) but also over the course of the 24-hour day (Robertson \& Dierker, 2003; Winje, Roislien, \& Froen, 2012). The fetal circadian rhythm is characterised by peak incidence of fetal activity in the evening and greater likelihood of quiescence during the day (Marsal, 1983; Patrick, Campbell, Carmicheal, Natale, \& Richardson, 1982). Although the drivers of diurnal variations in fetal movement patterns are not known, it has been suggested that they are under maternal control, due to fluctuations in maternal cortisol levels (Pillai et al., 1992). The 
reason for short-term or ultradian fluctuations in spontaneous fetal activity may reflect stimulation and adaptive interaction with the environment (Robertson \& Dierker, 2003).

\subsubsection{Fetal behavioural states}

Infant behaviours are increasingly recognised as having their origins in fetal life (Eispeiler, Marschik, \& Prechtl, 2008). Cycling through periods of rest and activity in the near-term fetus has been noted to be very like the behavioural-state cycling that occurs in infants. In infants it is recognized that there are five behavioural states; Quiet sleep (S1), active/rapid eye movement (REM) sleep (S2), quiet awake (S3), active awake with crying (S4) and active awake without crying (S5) (Nijhuis, Prechtl, Martin, \& Bots, 1982). The ability to visualise the fetal eye on ultrasound made way for study of fetal behavioural states (Pillai \& James, 1990). Nijhius and colleagues were the first to describe behavioural states in the fetus, naming these states $1 \mathrm{~F}$ through to $4 \mathrm{~F}$ to correspond with the neonatal behavioural states (Nijhuis et al., 1982). Pillai and James (1990) later questioned the existence of state 3F (quiet awake), describing the existence of three behavioural states, one quiescent $(1 \mathrm{~F})$ and two active (2F and $4 \mathrm{~F})$, in their realtime ultrasound study of 80 low-risk fetuses (Pillai \& James, 1990). Whether or not fetal active/wake cycles are strictly analogous to those in infancy is contestable. However, what is certain is that this episodic cycling occurs and its development at term is considered an indication of normal maturation of the neurological system.

\subsubsection{Fetal breathing movements}

Fetal breathing movements were the subject of much interest in the 1970 s and 1980s, after they were observed to be decreased in hypoxic fetuses (Marsal, 1983). In 1980 Manning proposed a test of fetal wellbeing, incorporating three fetal movement parameters (fetal breathing, tone and gross body movement), alongside liquor volume estimation and CTG, calling this test the fetal biophysical profile (BPP) (Manning, Platt, \& Sipos, 1980). Observational studies suggested fetal death rates were reduced when this test was used (Platt et al., 1985) and the test is in widespread clinical use today. However, successive Cochrane reviews of 
data from randomised controlled trials conclude that use of BPP does not reduce incidences of fetal death or low Apgar score, but is associated with a significant increase in induction of labour and caesarean section (Lalor, Fawole, Alfirevic, \& Devane, 2008).

\subsubsection{Ultrasound-observed fetal movements and pathology}

Identification of gestational ages at which certain movement patterns occur allowed a beginning of understanding of fetal neurological development. In the infant, abnormal general movement patterns have been shown to be effective predictors of brain damage (Cioni et al., 1997; Ferrari et al., 1997). Under pathological conditions the fetus might be delayed in demonstrating movement patterns appropriate for their gestational age or regress to an earlier stage of development (Marsal, 1983). Following the identification of fetal behavioural states by Nijhuis and colleagues (1982), a number of studies demonstrated delayed state organisation in association with pregnancy pathologies, including the growthrestricted fetus (Sival, Visser, \& Prechtl, 1992a; van Vliet, Martin, Nijhuis, \& Prechtl, 1985) and fetuses of insulin-dependent diabetic women (Dierker, Pillay, Sorokin, \& Rosen, 1982).

Neurological studies looking at alterations in quality and organisation of fetal movements in the context of pathology demonstrate that quality of fetal movements is as important a consideration as quantity, if not more so (Reissland \& Francis, 2010; Sival et al., 1992b). The healthy fetus displays a varied repertoire of complex movements. In the compromised fetus these movements become sluggish and stereotyped (Martin, 2008), whilst jerky or spastic movements (a normal feature of early third-trimester gestations) persist later in pregnancy in fetuses with neurological impairment.

Quality of fetal movements has also been shown to be altered in pregnancy complicated by hypertension. In a study of 21 hypertensive pregnant women compared with 22 normotensive controls, fetuses of hypertensive women displayed fewer body movements, lower magnitude of fetal heart rate accelerations and decreased coupling of cardiac and movement responses (Warner, Hains, \& Kisilevsky, 2002). Changes in fetal behaviour appear to occur 
prior to ultrasound evidence of fetal growth restriction (Kurjak, Talic, Honemeyer, Stanojevic, \& Zalud, 2013).

\subsubsection{Amniotic fluid}

Reduced amniotic fluid has also been shown to have an effect on fetal movement quality (Bekedam et al., 1985; Sival et al., 1990, 1992a). In 19 cases of premature rupture of membranes, there was a decrease in speed of movements with a moderate reduction of liquor, whilst a reduction in the amplitude of fetal movements was also seen where the liquor volume was severely reduced (Sival et al., 1990). Reduced amniotic fluid is an indication of placental insufficiency. However, in this study the effect appeared to be independent of fetal condition, and related specifically to the volume of amniotic fluid.

\subsubsection{Summary}

Present scientific understanding of normal fetal movement has largely been derived from ultrasound studies. Fetal development in relation to movement is characterised by increasing organisation of movements as pregnancy advances. At term the healthy fetus exhibits smooth, co-ordinated movements, with behavioural state changes. It has been proposed that observation of fetal movement may be used to assess fetal neurological status, but work towards developing such tools is only in its early stages. Quality of fetal movements is emerging as a better indicator of normal fetal development than quantity.

\subsection{Section Two: Maternal perception of fetal movements}

Maternal perception of fetal movement varies between women and over the course of pregnancy. The following section considers available literature on movements as perceived by the pregnant woman; correlation between ultrasound- 
observed movements and those perceived by the woman, as well as factors thought to influence maternal perception.

\subsubsection{Correlation between maternally-perceived fetal movements and objective detection}

The premise for investigation of correlation between maternal perception of fetal movements and objective measures is that fetal movements decrease in the context of fetal compromise and that perception of reduced movements by the mother is consistent with an actual reduction in movements and therefore predictive of compromise (Froen, 2004). Various methods for objective detection of fetal movements have been employed including piezoelectric crystals, analysis of fetal heart rate accelerations and real-time ultrasound. The earliest study of correlation between maternal perception of fetal movements and objective measurement reported a correlation of $87 \%$ (Sadovsky et al., 1973). Subsequent studies confirm that maternal perception of fetal movements correlates well with movements detected objectively, although with lower rates in the range of $35 \%$ to 56.4\% (Gettinger et al., 1978; Hertogs, Roberts, Cooper, \& Griffen, 1979; Hijazi et al., 2010; Lowery, Russell, Wilson, Walls, \& Murphy, 1995; Schmidt, Cseh, Hara, \& Kubli, 1984).

Although correlation between maternally-perceived and objectively recorded fetal movements is generally good, there is large inter-individual difference(Gettinger et al., 1978; Hertogs et al., 1979; Schmidt et al., 1984). In Gettinger and colleagues' (1987) study of 40 pregnant women, $40 \%$ of movements recorded on ultrasound were perceived by the pregnant woman, but between subjects accuracy ranged from $0-94 \%$. In that study, three subjects felt no movements at all despite movements being observed on ultrasound (Gettinger et al., 1978).

In a study of 20 women between 32 and 43 weeks gestation $35 \%$ of movements observed on ultrasound were perceived by the women overall but with similar findings in regard to inter-individual variation (Hertogs et al., 1979). In that study subtle movements such as fetal breathing movements and hiccups were 
correctly identified by some subjects, whilst others perceived very few movements despite frequent and at times large movements being seen on ultrasound (Hertogs et al., 1979). More recently Hijazi and colleagues reported correlation of maternal perception and ultrasound observation of $35.8 \%$, but a range of sensitivity to movements of $14-55 \%$ amongst 14 subjects (Hijazi et al., 2010).

Some types of movements, however, are easier for pregnant women to detect. These include gross body movements (Schmidt et al., 1984), movements involving more body parts (Hertogs et al., 1979; Hijazi et al., 2010; Rayburn, 1980; Schmidt et al., 1984), movements of longer duration and those involving contact with maternal structures (Hijazi et al., 2010).

Much of the variation in fetal movement perception is considered to simply represent idiosyncratic differences, which is important to acknowledge for clinicians. Whilst frequent fetal movement reported by a mother can be assumed to reflect actual movements, those women who perceive very few movements do not necessarily have an inactive baby.

All methods of fetal movement detection have limitations. Fetal movements are three-dimensional and techniques used in the above studies have generally employed one or two ultrasound transducers focusing on the trunk and one or two quadrants of the uterus. Some studies report 'false positives', that is movements reported by the women but not confirmed by ultrasound (Gettinger et al., 1978; Hijazi et al., 2010; Schmidt et al., 1984). It is possible some of these 'falsepositive' movements are actual movements that have occurred outside the ultrasound beam (Hijazi et al., 2010; Lowery et al., 1995).

Variation in reported correlation rates between maternally perceived and observed fetal movements likely reflect techniques used for objective measurement and subject sample sizes. Regardless of methodology, maternallyperceived fetal movements can be assumed to correlate with actual fetal movements at least a third of the time. The potential clinical significance of maternally-perceived fetal movements is not diminished by its moderate correlation with ultrasound-observed movements. Movements more likely to be detected by the mother include large movements and synchronous movements involving many body parts (Hijazi et al., 2010; Rayburn, 1980; Schmidt et al., 
1984). These vigorous movements are likely to be correlated with fetal wellbeing. Lower amplitude of fetal movements has been described in the context of reduced liquor volume (Sival et al., 1990), while growth-restricted fetuses perform fewer complex co-ordinated movements (Bekedam et al., 1985; Sival et al., 1992a).

The considerable variation between pregnant women in levels of fetal activity reported makes the establishment of normal and concerning levels of fetal movement difficult. For the most part studies examining maternal perception of fetal movements have been too small to reliably attribute variation in sensitivity to movements to maternal characteristics, although some characteristics are covered here.

\subsubsection{Parity}

First fetal movements perceptible by the mother are generally reported between 16 and 20 weeks, although there is considerable variation in this, with some women reporting fetal movements as early as seven weeks gestation and as late as 30 weeks (Raynes-Greenow et al., 2013). Parous women are able to identify movements earlier than women in a first pregnancy (Gillieson et al., 1985). It is not known whether parity itself increases sensitivity to fetal movements or if earlier quickening in parous women is an artefact of learning to identify the sensations. In a study of the influence of parity and placental site on quickening in 112 women, a significantly lower mean gestational age of quickening was seen in parous women, with those of parity greater than two reporting earlier quickening than those with one previous birth (Gillieson et al., 1985). Increasing gestational age and increasing parity were associated with increased accuracy in identifying fetal movements by Schmidt and colleagues (1984). Gillieson and colleagues (1985) determined that range in timing of quickening made it unreliable as an estimation of gestational age and that placental location had a greater influence on timing of quickening than parity (Gillieson et al., 1985).

\subsubsection{Placental location}


Anterior placenta, where the placenta is positioned at the front of the uterus, inhibits perception of fetal movements enough to delay quickening by a mean of seven days (Gillieson et al., 1985). However, the extent to which placental location impacts on perception of fetal movements later in pregnancy is difficult to determine. Incidence of anterior or posterior placental location is roughly equal, with $30-40 \%$ of placentas being anteriorly situated and the same proportion posterior whilst the remainder are fundal, lateral or low-lying (Gillieson et al., 1985; Kalanithi et al., 2007). A number of studies reported no statistically significant association between anterior placenta and reduced maternal perception of fetal movements (Gettinger et al., 1978; Hertogs et al., 1979; Schmidt et al., 1984). But these studies were small. Another study found an effect that diminished with advancing gestation (Neldam \& Jessen, 1980). Hijazi and colleagues reported that women were significantly more likely to identify fetal movements that involved contact with the uterus when compared to those that only contacted the placenta (Hijazi et al., 2010). Increased distance between fetal parts and the mother's abdominal wall has been proposed as an impeding factor in maternal perception of fetal movements (Tuffnell et al., 1991). In a survey of 78 women presenting with decreased fetal movements $72.2 \%$ were found to have anterior placenta (Fisher, 1999a). The clinical significance of reduced fetal movements in pregnancies with anterior placental location remains to be determined. One reviewer has warned that pregnancy characteristics that are constant should not be used to explain away a reported reduction in movements.

\subsubsection{Maternal obesity}

There is increased reporting of reduced fetal movements amongst obese pregnant women (Tveit et al., 2009). Maternal obesity is associated with increased risk of pregnancy complications, such as hypertension, gestational diabetes, disorders of fetal growth and increased risk of stillbirth (Stacey et al., 2011b). Despite these associations, the majority of obese women with decreased movements have a normal outcome, suggesting that fetal concerns may not be the major influence in decreased movement perception amongst obese women (Tuffnell et al., 1991). 
Tuffnell and colleagues (1991) have suggested than maternal obesity might interfere with perception of fetal movements according to similar mechanism as anterior placental location. One study found maternal perception of fetal movements decreased with increasing maternal weight but not with increasing maternal abdominal wall thickness (Lowery et al., 1995). The clinical significance of decreased fetal movements in overweight or obese women remains uncertain and warrants exploration, particularly given that maternal obesity is not only a risk factor for poor outcome but is also increasingly common amongst the childbearing population.

\subsubsection{Term gestation}

Frequency of fetal movements perceived at term is a controversial issue. Numerous sources have identified a slight reduction of movements at term. However, others deny any reduction. The issue is problematic in that women are often falsely reassured about concerns of reduced fetal movements at term by the belief that the change is a normal one for the stage of pregnancy. Kick-counting studies have identified that maternally-perceived fetal movements plateau around 28 weeks and then reduce slightly at term (Kuwata et al., 2008; Moore \& Piacquadio, 1989; Pearson \& Weaver, 1976). The change in fetal activity at term is likely to reflect neurological development of the fetus. Fetal activity at term is not well described, limiting useful information exchange between the woman and her care provider at term. Qualitative aspects of fetal movement perception at term have perhaps been under-investigated.

Two recent studies have begun to address this issue (Rådestad \& Lindgren, 2012; Raynes-Greenow et al., 2013). In an Australian survey on maternal perception of fetal movements, pregnant women described early fetal movements as 'soft', 'gentle' and 'light', later movements became 'stronger', 'bigger', 'harder' and might include jerky, spastic-type movements or rhythmic hiccups. At term, women commonly report stronger movements as compared to earlier in pregnancy with fewer kicks and jerks and more squirms and stretches (Raynes-Greenow et al., 2013). Women use a range of terms to describe the movements, including pushing, rolling, wriggling and pulsing. Use of the term 'kick' 
as a catch-all term for fetal movements might be problematic, as types of movements perceived by pregnant women are diverse and only a portion might be described by women as kicks, particularly at term when space is reduced and pushing, stretching or rolling movements dominate the pattern (Rådestad \& Lindgren, 2012). Thus 'kicks' may be reduced but movements overall may not. Improved understanding of normal fetal movements as perceived by the woman at term is particularly important, as both risk of compromise and potential for unwarranted intervention are increased at this time.

\subsubsection{Fetal position}

Fetal position may have an impact on perception of fetal movements. In a study of women unable to feel fetal movements seen on ultrasound, $80 \%$ of fetuses were in an anterior position, that is with their back toward the mother's front (Fisher, 1999a). This observation would fit with Tuffnell's (1991) assertion that some contact with maternal structures at the anterior abdominal wall is required for fetal movements to be perceived by the mother and might also explain the higher proportion of benign complaints of decreased fetal movements at term, when the fetus is more likely to have adopted an anterior position prior to birth.

\subsubsection{Smoking}

Women reporting decreased fetal movements are more likely to be smokers (Tveit et al., 2009). It has been established that fetuses of women who smoke are less active than their smoke-free counterparts and have a higher proportion of sluggish movements in relation to brisk movements (Habek, 2007). Maternal smoking is also associated with intra-uterine growth restriction, placental pathologies and with intra-uterine death (Coppens, 2001). The fetus exposed to maternal smoking is periodically deprived of oxygen and may also be deprived of nutrients where smoking has impacted on placental architecture (Habek, 2007). Decreased fetal movements in the smoke-exposed pregnancy may be an adaptive response of the fetus prioritising limited energy supplies for growth as opposed to 
movement. There is no known reason why smoking might inhibit perception of fetal movements per se, suggesting reports of decreased movements are likely to indicate fetal effects from smoking.

\subsubsection{Maternal meals}

There are conflicting reports about fetal activity in association with maternal meals. Some authors have reported that fetal movements are increased following meals (Eller, Stramm, \& Newman, 1992; Miller, Skiba, \& Klapholz, 1978), but subsequent studies refuted this (Bocking, Adamson, Carmicheal, Patrick, \& Probert, 1984; Druzin \& Foodim, 1986; Patrick et al., 1982). Endocrine studies have shown that fetal movements are increased in the context of hypoglycaemia in pregnant women with diabetes (Holden, 1984), whilst fetal movements in normal pregnant women are decreased in the context of hyperglycaemia (Edelberg, Dierker, Kalhan, \& Rosen, 1987). Direct maternal reports of fetal activity around meals have not been sought. In a cross-sectional survey of 156 women with singleton pregnancies, $12 \%$ of participants reported increased fetal movements in association with hunger or eating (Raynes-Greenow et al., 2013).

\subsubsection{Amniotic fluid volume}

Women who report decreased fetal movements are more likely to have decreased amniotic fluid volume (Ahn et al., 1987). Amniotic fluid volume is an indicator of placental function and the correlation between decreased fetal movement and reduced liquor volume suggests placental insufficiency. However, amniotic fluid levels might also have a mechanical influence on fetal movement perception. Fetal movements are reduced in speed and amplitude in the context of rupture of membranes and low fluid levels (Sival et al., 1990), which may translate into a perception of reduced movement by the pregnant woman. At term women may perceive movements to be more defined, potentially a consequence of the normally lower levels of liquor at term as compared to earlier in the pregnancy. 


\subsubsection{Time of day}

Pregnant women describe the evening as the busiest time for fetal activity and for this reason evening is commonly suggested as an ideal time for fetal movement counting (Moore \& Piacquadio, 1989). In a British audit of pregnant women's impressions of fetal activity according to time of day, evening was the most likely time for movement (Fisher, 1999b). An Australian survey also reported increased likelihood of fetal movement as the day progressed, with the most movement reported by women occurring in the evening (Raynes-Greenow et al., 2013). It has been established by ultrasound studies that fetal movements have a diurnal pattern (Marsal, 1983; Pillai et al., 1992). The diurnal fetal movement pattern is characterised by increased activity in the evenings and increased quiescence in the mornings, which is consistent with maternal accounts of fetal activity patterns. Drivers of the fetal circadian rhythm are not known. However, it has been suggested that maternal cortisol may play a role (Marsal, 1983). Despite the establishment by objective means of increased fetal activity in the evening, maternal reports of increased activity in the evening are still commonly ascribed to maternal inattention to movements during the day (Raynes-Greenow et al., 2013; Whitworth, Fisher, \& Heazell, 2011). The clinical significance of the fetal circadian rhythm is unknown, but is likely to be an under-appreciated aspect of the maternal account of fetal movements.

\subsubsection{Maternal position}

Minors and Waterhouse (1979) investigated maternal perception of fetal movements according to whether the woman was standing, sitting or lying over the course of the day, concluding that fetal movements are more perceptible lying when compared to sitting, and sitting when compared to standing (Minors \& Waterhouse, 1979). This study involved six participants, and a major limitation to the study is that just three positions were recordable by participants, all of them static. Times of day in which the woman would find herself sitting could be expected to be for meals and in the evening. These are times when fetal activity is known to be increased. Minors and Waterhouse's (1979) findings of variation in 
movement-perception according to maternal position might therefore be confounded by kinaesthetic factors, maternal mealtimes or by the fetal circadian rhythm. Despite these limitations, this 1979 study remains a frequently cited reference in relation to maternal position and fetal movement perception.

\subsubsection{Summary}

Fetal movements perceived by women correlate well with those observed by ultrasound. Strong, complex and gross body movements are even more accurately identified by pregnant women. Patterns of fetal activity indicative of normal neurologic development, such as a subsiding of jerky movements at term and emergence of circadian rhythm, are also readily identifiable by pregnant women. These correlations support the notion of the pregnant woman's experience of fetal activity being a qualitative one.

Despite this there are many gaps in knowledge of factors influencing maternal perception of fetal movements. The influence of maternal obesity, placental location and term gestation in particular demand further exploration.

\subsection{Section 3. Monitoring and assessing fetal movements.}

The following section considers key fetal movement monitoring studies published to date, including attempts to define normal and pathologic levels of fetal movement, acceptability of monitoring to women, management of pregnancy where fetal movement concerns have been raised, and practitioner views on fetal movement assessment.

\subsubsection{Establishing normal and pathological levels of fetal movement}

The development of fetal movement monitoring tests has usually involved first attempting to establish a definition of normal fetal activity. Numerous attempts to establish fetal movement levels for monitoring purposes have been made. Pearson and Weaver (1976) collected fetal movement count charts from 61 pregnant outpatients from 32 weeks until term, who subsequently delivered infants 
above the $25^{\text {th }}$ centile (Pearson \& Weaver, 1976). Participants recorded a median value of 90 kicks per 12 hours of counting (9am to $9 \mathrm{pm}$ ) at 32 weeks, dropping to 50 per 12 hours at 40 weeks. Only $2.5 \%$ of women had daily movement counts of fewer than 10 movements in 12 hours at any gestation. A count of fewer than 10 movements per day was found to be positively correlated with fetal compromise and fetal death in that study. The authors declared an important finding of their study was that more than 10 movements per day assured a normal outcome. Their movement-counting chart, the Cardiff 'Count to 10 Kick Chart', subsequently entered widespread usage and the 10 kicks in 12 hours definition persists today (Unterscheider \& O'Donoghue, 2012).

Later studies have reported somewhat higher levels of normal fetal movement. Moore and Piacquadio (1989) established levels of fetal movements in a pilot study of 100 women, reporting that the time taken to count 10 movements while resting on their left side in the evening was 20.9 minutes (+/18.1 minutes) (Moore \& Piacquadio, 1989). Within 90 minutes $99.5 \%$ of women normally counted 10 movements in this way, therefore 2 hours was identified as an alarm limit, being more than 5 standard deviations from the mean.

Kuwata and colleagues (2008) established a reference value for frequency of fetal movements by asking 705 low-risk primiparous women to record the time it took to count 10 movements at a time of day when their baby was normally active. The median time to count 10 movements was 10 minutes at 32 weeks, and 14.8 minutes at 40 weeks (Kuwata et al., 2008). In a prospective cohort study investigating fetal movement patterns in normal low-risk pregnancies at term, Winje and colleagues (2011) reported the mean count-to-10 time in 582 normal pregnancies was 10 minutes, in keeping with that reported by Kuwata and colleagues (Winje et al., 2011).

Variation in normal and abnormal ranges in fetal movement counts reported are likely to relate to methodology employed, including risk status of the pregnancy, time of day of counting and number of participants. It is clear that later studies involving higher numbers of low-risk participants have reported considerably higher numbers of movements as normal than was first reported in early papers proposing fetal movement counting, making it a matter of some concern that the relatively low number of movements espoused by Pearson and 
Weaver in 1976 as reassuring is still in common usage. Qualitative aspects of fetal movement as perceived by the mother have not been investigated formally in fetal movement monitoring tools.

\subsubsection{Fetal movement monitoring methods}

More than a dozen different methods of fetal movement monitoring have been devised, which can be broadly divided into two groups, the Sadovsky method which involved counting movements three times a day, an hour in the morning, an hour at midday and an hour in the evening, and the Cardiff 'Count-toTen Kick Chart' method, derived from Pearson and Weaver's daily fetal movement count (Froen, 2004). Later modifications of Sadovsky's method involved shorter movement-counting times and attempts to establish individualised norms.

\subsubsection{Fetal movement counting studies}

A number of early studies supported fetal movement monitoring as beneficial (Liston, Cohen, Mennuti, \& Gabbe, 1982; Rayburn, Zuspan, Motley, \& Donaldson, 1980; Sadovsky, Yaffe, \& Polishuk, 1974). But these were small, and larger studies were called for. In 1980 the results of a Danish study evaluating fetal movement counting using Sadovsky's method versus normal care were published in The Lancet. The study involved 2225 women and reported eight perinatal deaths in the control group and none in the group allocated to fetal movement counting (Neldam, 1980). Although this study was a randomised controlled trial, the randomisation process (using odd or even booking numbers) was considered methodologically flawed by some reviewers (Mangesi et al., 2007).

In the '70s, the Cardiff 'Count-to-Ten Kick Chart' was introduced to one of two consultant units at Liverpool Maternity Hospital, opening up the opportunity to compare populations at the same institute routinely using fetal movement counts with those who didn't. The study period covered four years and encompassed more than 20,000 births. On comparing outcome data, the authors reported that there was no difference in stillbirth rates between the two units, concluding that the charts offered no benefit (Lobb, Beazley, \& Haddad, 1985). However, the chart 
was in use in both units, for high-risk women only in Unit B and for all women after 28 weeks in Unit A. During the study period a significant drop in stillbirths was seen, from 15.6 per 1000 in the four years prior to the study period, to 6.5 per 1000 during the study period (Lobb et al., 1985). A reported reduction in fetal deaths in the course of conducting a fetal movement study appears to be the case regardless of fetal movement monitoring method.

In New Zealand a retrospective study comparing stillbirth rates over two consecutive 20 month periods, each involving more than 8000 births at National Women's Hospital revealed a significant decrease in stillbirth rates and perinatal mortality rates (from 10.83 per 1000 births to 8.21 per 1000 births) following the introduction of a fetal movement counting card in the second 20 month period. In that study the number of unexplained stillbirths decreased from 48 to 27 in period following the intervention (Westgate \& Jamieson, 1986).

In 1989 two very different fetal movement counting studies were published, one of which was to halt advancement in study of fetal movement assessment almost irrevocably. Moore and Piaquadio (1989) conducted a prospective study comparing perinatal mortality rates in two seven-month time periods at an American Naval Hospital, one prior and one subsequent to a fetal movement counting intervention. During the study period fetal mortality fell from 8.7 to 2.1 per 1000. However, this decrease was accompanied by increased induction of labour and emergency caesarean sections for fetal distress (Moore \& Piacquadio, 1989). Although the results of this study were very promising, the study has not been repeated. Subjects were military dependants or active servicewomen who had high levels of compliance with antenatal care and it cannot be determined whether the method of counting would be acceptable to other populations. Increased rates of induction of labour and of emergency caesareans could be expected to carry a risk of iatrogenic harm. Large-scale evaluation would be required to determine the impact of this increased intervention. Further, the subjects were instructed to lie on their side to count fetal movements every evening, and to present that evening for assessment should it take them longer than two hours to count 10 movements. The definition of 10 movements in two hours is commonly used in the United States following this study but the specific method of daily evening counts is not promoted, making it debatable whether the definition has any value in other settings. 
That same year in The Lancet, the results of a large multi-centre randomised controlled trial comparing fetal movement counting with routine care was published (Grant et al., 1989). This was a highly anticipated study, coming as it did at a time when the importance of evidence-based medicine and particularly the large randomised controlled trial had come into focus for maternity care. More than 68,000 participants were enrolled at hospitals across Europe into counting and/or routine care groups. At its conclusion the authors reported no significant difference in stillbirth rates between the two groups (Grant et al., 1989). Since that time, evidence-based guidelines have been unable to recommend fetal movement counting, and (aside from a recent resurgence) very few fetal movement monitoring studies have been conducted in the subsequent 20 years since publication of that study.

The study has since come under criticism for a number of methodological weaknesses, including contamination via the cluster randomisation process, which meant women who were not counting were likely to come into contact with those counting, and indeed in many cases control women were advised to count if their obstetrician felt it might be beneficial. Participants allocated to counting were given a Cardiff 'Count-to-Ten Kick Chart' and instructed to count fetal movements from 9.00 a.m., noting the time at which they counted 10 movements. Participants who failed to count 10 movements by 9.00 p.m. on two consecutive days were to present for assessment. This alarm limit could be considered extreme and it is possible women who might otherwise have presented with fetal movement concerns were falsely reassured by such a low limit of 'normal' movement, meaning cases were missed.

Although no difference was reported in death rates between the control group and the study group, stillbirth rates dropped during the period of the study, which authors attributed to the Hawthorne effect (Grant et al., 1989). The authors themselves concluded that their results 'do not indicate that maternally perceived reduction in fetal movements is clinically unimportant' but rather that monitoring in the method tested conferred no benefit when compared to usual care (Grant et al., 1989, p.348). A Cochrane review has pointed out that no high-quality randomised trial has compared fetal movement counting to no fetal movement counting (Mangesi et al., 2007). 
In a novel approach to identifying low levels of movement for screening purposes, 1786 women recorded fetal movements prospectively on charts which were analysed following birth to determine whether movement numbers recorded during pregnancy were predictive of poor outcomes. Later analysis determined an alarm of fewer than 10 kicks in two hours failed to detect $95 \%$ of cases of poor outcome, whilst an alarm limit of 25 or 35 minutes (at term) had a high false positive rate (Winje et al., 2011). The study authors suggest analysis of movement patterns amongst at-risk sub groups might be beneficial, as quality and force of movements may be important but has been under-studied (Winje et al., 2011).

\subsubsection{Acceptability of fetal movement monitoring methods: compliance and untoward effects}

Possible negative impacts of fetal movement screening on women need to be considered. Formal fetal movement counting has not been proven to reduce mortality, but may contribute to increased intervention and can cause anxiety in pregnant women (Mangesi et al., 2007). Fetal movement monitoring methods evaluated to date have largely involved counting movements. In two studies of the psychological effects of counting on women, counting movements was associated with increased feelings of attachment to the fetus (Mikhail et al., 1991), whilst another showed high levels of compliance ( $90 \%$ of women counted for $95 \%$ of days) and deleterious psychological effects (Liston, Bloom, \& Zimmer, 1994). In the Grant trial, women allocated to counting reported they felt very or quite anxious in late pregnancy as compared to controls (difference in mean 2.0 per 100 women, $95 \% \mathrm{Cl} 1.8$ - 5.8). However, women in the counting group were also more likely to feel 'in control' and confident. A third of women in both groups reported anxiety about fetal movements at some time during late pregnancy. Whether or not women would have remained feeling confident had they known the counting method was ineffective remains to be seen.

\subsubsection{Management of reduced fetal movements}

Assessment of the woman who presents with concerns about fetal movements is challenging. Where there are overt signs of compromise, action is 
indicated, but where all appears well the path is less clear. In the majority of cases (70\%) where there is a single presentation for reduced fetal movements, the outcome is good (Whitworth et al., 2011). Unwarranted intervention in these pregnancies risks iatrogenic harm (Olesen \& Svare, 2004).

Conversely, inadequate assessment of pregnancies with a complaint of decreased fetal movements has been identified as a failure in audit of stillbirths. Possible modes for fetal assessment in the context of decreased movements include auscultation of the fetal heart, symphysial fundal height measurement, fetal weight estimation, liquor volume assessment, umbilical artery and uterine artery doppler velicometry, biophysical profile and formal fetal movement counting (kick charts).

Historically, assessment of maternal complaints of decreased fetal movements has often been limited to simple auscultation of the fetal heart (Froen, 2004). Auscultation of the fetal heart confirms fetal life, but has no predictive value in terms of later outcome of pregnancy (NICE, 2008), as one reviewer points out 'the last thing the fetal heart does before it stops beating, is to beat.' (Froen, 2004 P15.) CTG monitoring is the most common approach to assessment of decreased fetal movements and most practitioners view a normal CTG as sufficient assessment (Hofmeyr \& Novikova, 2012). A CTG that displays the usual features of a reassuring trace excludes any acute hypoxic event, but once again has poor specificity as a test of ongoing fetal wellbeing. A chronically hypoxic fetus may compensate for its precarious position for some time, continuing to display movements and heart-rate accelerations, and therefore a normal CTG. However, subsequent decompensation therefore cannot be excluded (Heazell, Sumathi, \& Bhatti, 2005; Martin, 2008). In a Cochrane review on the efficacy of antenatal cardiotocography for fetal assessment, it was concluded that there is no clear benefit of CTG for improving outcome (Pattison \& McCowan, 2010). For this reason some guidelines advocate further assessment where the CTG is normal but the woman still feels the movements are reduced (Whitworth et al., 2011).

Evidence for the use of umbilical artery Doppler or uterine artery Doppler in the assessment of pregnancies with reduced fetal movements is limited. Dubiel and colleagues (1997) compared CTG with umbilical artery Doppler in assessment of low-risk women who complained of decreased fetal movements, and found that 
CTG was a better predictor of mortality (Dubiel, Gudmundsson, Thuring-Jonsson, Maesel, \& Marsal, 1997). The biophysical profile incorporates fetal movement assessment on ultrasound alongside observation of other fetal biophysical activities on the basis that such activities are less likely to be carried out in the hypoxic fetus. A systematic review of BPP to assess women with high-risk pregnancies concluded that BPP did not confer any benefit but was associated with increased intervention (Lalor et al., 2008). Much like CTG the biophysical profile can provide an assessment of fetal wellbeing at the time of the examination, but cannot provide an estimate of fetal reserve (Dayal, 1999).

Assessment of amniotic fluid volume is advocated in the work-up for reduced fetal movements as reduced liquor volume is associated with placental insufficiency. However, methods of assessment can be unreliable, and in the case of amniotic fluid volume (AFI), is associated with increased rates of induction of labour without improvement in outcomes (Nabhan \& Abdelmoula, 2008).

A key benefit in assessment of complaints of reduced fetal movements is increased identification of small-for-gestational-age (SGA) fetuses, either by symphysial fundal height measurement, ultrasound or both (Tveit et al., 2009). Careful measurement of symphysial fundal height has been shown to have greater specificity than a single ultrasound assessment in predicting IUGR at birth (Heazell et al., 2005). Use of this simple method can reduce unnecessary ultrasound scans and may also prevent stillbirth where it is used alongside customised fetal growth charts (Gardosi, Giddings, Clifford, Wood, \& Francis, 2013).

Inadequate assessment of maternal reports of reduced fetal movements has been implicated in preventable stillbirths, but optimal assessment remains to be determined. Stringent assessment of cases of decreased fetal movements has not been shown to lead to improved outcomes (Skornick-Rapaport, Maslovitz, Kupferminc, Lessing, \& Many, 2011), and may be associated with increased intervention (Heazell et al., 2013). There has notably been no large randomised controlled trial testing fetal wellbeing assessment in the instance of decreased fetal movements (Hofmeyr \& Novikova, 2012). Further research is needed to guide practice in this area. 


\subsubsection{Practitioner views and current approaches to FM monitoring}

A number of studies have investigated practitioner views in relation to fetal movement screening and assessment. The majority of midwives and obstetricians believe fetal movements are important and enquire about fetal movements as part of antenatal assessment. But beyond simple enquiries after fetal movement at antenatal visits, approaches to screening and assessment vary considerably. When defining reduced fetal movements, a subjective impression by the pregnant woman of fewer movements in the last 24 hours is preferred by obstetricians in New Zealand and Australia (Flenady et al., 2009), whilst in a survey of midwives and obstetricians in the Republic of Ireland, the most frequently provided definition of decreased fetal movements is fewer than 10 movements in 12 hours (Smith, Begley, \& Devane, 2014).

When assessing a complaint of reduced fetal movements, the majority of practitioners would perform a CTG (Flenady et al., 2009; Heazell et al., 2008; Smith et al., 2014). In England 82\% of surveyed midwives and obstetricians would also measure symphysio-fundal height, whilst fewer than $40 \%$ would in Ireland (Unterscheider, Horgan, Greene, \& Higgins, 2010). Fewer than 5\% of practitioners in England would institute a kick chart (Heazell et al., 2008), whilst $35 \%$ in Australia or New Zealand would (Flenady et al., 2009) and in the Republic of Ireland between $47 \%$ and $62 \%$ of practitioners would do so (Smith et al., 2014).

Practitioners value, in principle, subjective perception of fetal movements by the pregnant woman but have little to guide them in terms of using this approach in practice. The great variation in approaches to fetal movement screening and assessment by health professionals reflects the lack of robust evidence to guide practice.

\subsection{Summary}

Health professionals have long acknowledged the importance of maternally perceived fetal movements, but continue to lack reliable tools for assessing normal fetal activity. Given the importance of normal fetal movement patterning and quality of movements to understanding of fetal neurology, it is regrettable that investigation of maternal perception of fetal activity to date has largely been limited 
to quantitative evaluation or simple kick-counting. A lack in cross-pollination of knowledge and ideas between disciplines with an interest in the fetus and the neonate has held back potential advancement in perinatal care. Maternally perceived fetal movements correlate well with those observed on ultrasound, particularly those movements that are larger or grouped. Such qualitative aspects of fetal movement perception may be important as large movements or coordinated groups of movements are likely to indicate fetal wellbeing. Maternal concern about a change in fetal movement pattern or a sense that 'something is not right' may well be a result of pregnant women's observations of qualitative changes in their baby's movements. 


\section{Chapter 3}

\section{Research method and rationale}

\subsection{Structure of chapter}

This chapter begins with the rationale behind the methodology selection, firstly by including an overview of the clinical problem prompting the investigation. Consideration is then given to qualitative research and the place of qualitative research in health. An introduction to qualitative description as a methodology and consideration of the methodology's fit with the research problem follows and finally the present study's design, methods of data collection and analysis are outlined.

\subsection{Methodology selection}

In order to focus on the perception of fetal movements by pregnant women, the research methodology of qualitative description has been chosen (Sandelowski, 2000). This choice is a pragmatic one. The brief of the researcher using qualitative description is to obtain a broad description of an event or phenomenon in the everyday terms of that event/phenomenon. Qualitative description is also embracing in variation, which is appropriate to the topic of maternal perception of fetal movements which is already understood to be varied. The first impetus to investigate fetal movements as a marker of fetal compromise was inspired by verbal reports from pregnant women that their babies moved less prior to intra-uterine fetal death (Sadovsky \& Yaffe, 1973). Yet, for the greater part, investigation to date has focused on quantification of fetal movements, with very little consideration of women's verbal accounts in either normal pregnancy or complicated pregnancy. Consideration of a qualitative problem demands qualitative means, and when little is known about a subject a broad-based approach is a sensible place to start.

\subsection{Maternal perception of fetal movements: A problem ripe for qualitative investigation}


Despite technological advancements in perinatal care in developed countries, unanticipated death or injury to the fetus continues to be a significant problem (Goldenberg et al., 2011). Maternal perception of decreased fetal movements is a specific indicator of fetal jeopardy (Frøen et al., 2008b). Changes in fetal movements that are worrisome for the pregnant woman often precede signs of fetal compromise detectable by other methods, opening up the possibility that with better screening methods compromised fetuses may be identified earlier. A significant shift has occurred in acknowledging the woman's subjective impression of reduced fetal activity as more important clinically than any numerical definition (Tveit et al., 2009). Yet the nature of maternally-perceived fetal activity has been under-investigated using qualitative means (Rådestad \& Lindgren, 2012; Raynes-Greenow et al., 2013). Quantitative definitions of normal continue to be used in antenatal care, which can be unsatisfactory for women, causing either anxiety or confusion. Qualitative aspects of fetal movement perception are valued by health professionals (Raynes-Greenow et al., 2013), but very little knowledge about these aspects exists on which to base discussions between pregnant women and health professionals. In particular, the nature of fetal movements at term is poorly defined (Stacey et al., 2011a), a belief that movements subside prior to labour can lead to delayed presentation and potentially avoidable death (Peat, Stacey, Cronin, \& McCowan, 2012; Rådestad, 2010); and lack of understanding of normal movements at this stage can put the woman and baby at risk of unnecessary intervention and iatrogenic harm.

For this study it was felt a return to the original source of early warning of fetal compromise - the verbal account of the pregnant woman - was required. As perception is inherently qualitative in nature, and qualitative aspects of fetal activity have been under-investigated and are potentially important, it was felt a qualitative approach must be used. At the time of planning this study no qualitative study of maternal perception of fetal movements had been published and very little to guide exploration. Therefore an inductive approach was called for. The woman's account specifically was sought and the methodology needed to embrace variation. The underlying interest was in the physiology behind maternal perception of fetal movements and fetal compromise, and whether the maternal account of normal fetal movements might provide guidance as to physiological understandings of fetal movement and fetal wellbeing. The approach therefore 
needed to be data driven and start with the woman. Qualitative description was identified as an appropriate methodology, with individual interviews the selected method. In order to better understand the rationale for these choices a discussion of qualitative research in health and an introduction to qualitative description as a methodology for use in health research will follow.

\subsection{Qualitative research}

Qualitative researchers are by degrees committed to a critical understanding of the positivist or quantitative paradigm. Positivism, or the school of philosophical thought holding that reality can only be understood through observation of things and deductive logic, arose in the eighteenth century largely as a reaction to the Church and mystical understandings of the world (Grbich, 2007). Scholars of the Enlightenment began to use mathematics and empirical observation to describe the world. Within medicine, such methods challenged practices based on centuries of teaching. French Physician Pierre Chatles Alexandre Louis, a pioneer of epidemiology, used careful observation of patients and tabulation of progress to 'prove' that bloodletting, a centuries old treatment for inflammatory conditions, was not of significant benefit and could be abandoned (Morabia, 1996). Louis held that there were certain laws of nature that were immutable and discoverable only by observation and testing. Positivists were concerned with the essential nature of things and a single objective reality that could be observed, quantified and proven by manner of objective testing (Malterud, 2001b). Objectivity and control of variables are essential elements of rigour when carrying out quantitative studies, with the randomised controlled trial being the epitome in methodology (Malterud, 2001b; Pope \& Mays, 1995).

Whilst the benefits of this view are considerable, the positivist paradigm has come to have an almost unassailable authority, such that evidence is not considered valid unless reached by such means. Science based on mathematics and experiment is termed 'hard science', whilst 'soft science' or the social sciences are considered less valid. Academics within the qualitative traditions question the notion of the single objective reality and point out that traditional scientists readily claim the high-ground of objectivity, but seldom make explicit 
their own moral and political viewpoints driving their work (Denzin \& Lincoln, 2011).

Qualitative researchers have long acknowledged that not all types of knowing can be arrived at via the randomised controlled trial and not all clinical questions are amenable to quantitative testing (Meadows, 2003). Clinicians employ tacit ways of knowing when applying their knowledge to patients. The generalised data obtained from large quantitative studies cannot always be easily applied to all patients due to their great variation. Consideration must also be given to the values of individuals and idiosyncratic responses. Donald Schon (2001) described this problem in his theory of 'reflection-in-action' where professionals bring 'artistry' to their work in order to make wise decisions in "indeterminate zones of practice - the situations of complexity and uncertainty" (Schon, 2001)(p. 189). The case of the pregnant woman presenting with concerns about her baby's movements is an excellent example: the woman has a sense that 'something is not right', and health professionals by and large acknowledge the importance of the woman's subjective impression but lack the tools and evidence for appropriate assessment and management of a subjective concern.

Social scientists reject the notion of immutable 'laws of nature' and instead understand phenomena to exist in a social milieu where meaning is contextual and subject dependant. The single objective reality is replaced by multiple and subjective realities (Hutchinson, 2001; Nicholls, 2009). The 'essential' replaced by the 'existential' if you will. Social scientists question whether true objectivity is really achievable, preferring to make explicit their own position when conducting research.

Generally speaking, the qualitative research process involves acknowledgement of the biographically situated author, who views the world through a set of beliefs contingent on their social context. Strategies of inquiry are developed according to the methodology that best fits the problem. Qualitative data is usually gathered by interviews, case studies or direct observation. The purpose is to represent the world in terms of the multiple interpretations humans make of their experiences (Denzin \& Lincoln, 2011). Analysis of data is conducted according to the traditions of the selected methodology, and is largely interpretive or theoretical. In terms of knowledge claims, the concept of generalisability used in quantitative research gives way to transferability. In transferability, a well- 
developed theory raised up out of qualitative data and thoughtfully and rigorously constructed should be sound enough to ring true for those working in similar contexts and so be transferable to other situations (Sandelowski \& Barroso, 2002).

\subsection{Qualitative research in health}

In health-care research, the quantitative approach is useful for understanding disease, but more inductive or interpretive methods are called for when it comes to understanding patients (Malterud, 2001b; Nicholls, 2009). Qualitative research can aid understanding of patient beliefs around health issues, for example an ethnographic study exploring mothers' perceptions of diarrhoeal disease amongst young children in Northern Thailand revealed that mothers believed diarrhoea was necessary to 'lighten the body' so that infants could progress to new development stages, such as standing or walking (p.965). The authors concluded that these views were deeply held and constituted the reason most mothers paid little attention to preventing or managing diarrhoea according to medical recommendations (Pylypa, 2009). Information of this type is useful for health-care providers and can only gained through in-depth qualitative interviewing (Morse, 2011).

Qualitative research in health, more so than in other disciplines, is pragmatic. Health-professional researchers seek to understand a problem better not just for understanding's sake but so that care provision might be improved. Health-care research is dominated by experimental studies, with a focus on treatment and cure of illness. Very often the person's experience of the health issue is 'back-staged'. Qualitative health research focuses more readily on the experience of the patient. However, the line between qualitative and quantitative, and their respective contributions to health, is not so easily discerned. Modern understanding of health, illness and disease has been built up from centuries of observation of symptoms and detailed case studies of typical and varying cases (Morse, 2011), a process that is certainly qualitative in nature. The notion that qualitative methods can be complementary to the traditional experimental approach (for example, by investigating patient views on a therapy or providing a preliminary understanding of a problem for later experimental study), is one that is 
embraced by qualitative health-care researchers - although for some, viewing qualitative research simply as a complement to the 'real' work of positivist science may be seen as minimising of the qualitative research act (Denzin \& Lincoln, 2011).

Likewise, to reduce the process of scientific discovery to its experimental phase is to do science itself a disservice. Traditional science is not only measurement and deduction but is also a creative endeavour. Scientific discoveries typically follow a process beginning with observation, followed by questioning and theorising, and finally testing of hypotheses. An example of this process within perinatal care is the discovery of the benefits of calcium supplementation in reducing the incidence and severity of pre-eclampsia. Belizian and Villar (1980) observed that Mayan Indians of Guatemala had very low incidence of pre-eclampsia and also enjoyed a diet rich in calcium due to a staple of corn dipped in lime juice (Kumar, Devi, Batra, Singh, \& Shukla, 2009). The hypothesis that calcium supplementation might be beneficial to pregnant women in preventing pre-eclampsia developed from such observations (Belizian \& Villar, 1980) and later testing has shown it to be so (Bucher et al., 1996).

Much has been made of the paradigm divide between qualitative and quantitative research, between the positivist and interpretivist schools, but the rift may have been overstated (Pope \& Mays, 1995). Malterud (2001b) emphasises that diversity is needed in medical research and that separatism and supremacy should be avoided if knowledge in the field is to be expanded. Taking a step back to empirical observation of fetal activity, via the pregnant woman, offers potential to generate new hypotheses around fetal responses to adverse intrauterine conditions and so advance stillbirth prevention efforts.

\subsection{Qualitative description}

The key elements of qualitative description relate to the methodology's brief of obtaining a broad description of an event or phenomenon in the everyday terms of that event/phenomenon (Sandelowski, 2000). The approach is simple but by no means simplistic. Qualitative description is a fundamental qualitative approach that 
is relatively light on theory, employs maximal sampling techniques and maintains a low-inference approach to data analysis. The facts of the case under investigation are reported in everyday terms rather than being filtered through complex theoretical frameworks (Neergaard, Olesen, Andersen, \& Sondergaard, 2009; Sandelowski, 2000).

Qualitative description is a qualitative research methodology that has arisen from within Nursing and was first described by Sandelowski (2000). This methodology was not invented by Sandelowski, as she asserts, but had been around for some time, often going by other names. Qualitative description is different from quantitative description and distinct from qualitative interpretation as I will show (Sandelowski, 2000; Thorne, Kirkham, \& MacDonald-Emes, 1997; Thorne, Reimer Kirkham, \& O'Flynn-Magee, 2004). In quantitative description, parameters for exploration are set out prior and analysed statistically, thereby limiting the potential for participant interpretation of events or discovery outside the pre-set area of investigation.

Qualitative research is generally described as entailing description, interpretation and theory (Giacomini, 2000; Malterud, 2001a; Sandelowski \& Barroso, 2002) Qualitative description has a heavier emphasis on description and holds back on interpretive leaps or deep theoretical penetration of the data. The methodology occupies a potentially unenviable position within the health research arena of being a fundamental qualitative approach, so-called 'soft' science, yet with its emphasis on description and relative atheoretical standpoint can at times be seen as a poor cousin of the heavily interpretive qualitative research agenda. In a setting where interpretation theory has 'surged forth to such an extent that there are hardly any competitors to its hegemony' (Giorgi, 1992 p. 199), qualitative description may be regarded as rather a low rung on the ladder of qualitative methodologies (Neergaard et al., 2009; Sandelowski, 2000). Yet the approach has a particular logical tension of its own, as I will show.

\subsection{Descriptive interpretive tension}

Qualitative research is alternatively described as 'empirical' (Giacomini \& Cook, 2000) and 'interpretive' (Malterud, 2001a). These concepts are not 
interchangeable and an understanding of them helps to appreciate where qualitative description fits within the paradigm. Empiricism is knowledge drawn from observation, or sense experience. In this broad sense traditional science is empirical in that evidence is collected via observations. The key difference between qualitative and quantitative empiricism is the deductive approach used by quantitative researchers to establish knowledge claims by refutation and the inductive or affirmative approach employed by qualitative researchers (Malterud, 2001a; Nicholls, 2009).

Qualitative description follows the logical empirical approach used by the naturalists. Naturalistic inquiry is used in both human and animal studies where the researcher studies the target phenomenon in its natural setting (Sandelowski, 2000). The intention is to be in the field and allow the subject to 'present itself as it would if it were not under study' (Sandelowski, 2000, p. 337). In qualitative description, meanings are clarified precisely as experienced, in the language of those experiencing the phenomenon, without contingent theoretical perspectives or assumptions. The biographically situated author is an accepted feature of qualitative research; however, in qualitative description the researcher takes a back seat, seeking to keep inference to a minimum. If qualitative research methodologies were considered on a continuum, qualitative description would be at the more traditionally scientific end. Variation both in sampling and data analysis is embraced in descriptive work. There is no need or intention to press on to a univocal position or force the data to fit a predetermined theoretical perspective. The subject of investigation is simply described in its variation, thereby being rendered as having a unique character. The objective of the present study is simply to provide a description of fetal movements as perceived by the pregnant woman, as a physiological phenomenon, as opposed to seeking an understanding of the essential nature or the social meaning of fetal movement perception for women.

Giorgi (1992) describes interpretation as being motivated by 'a situation of doubt, ignorance or unclarity' (p. 122), and goes on to suggest that, were interpretive researchers to outline explicitly where they departed from the data in order to fill out their inductive theory, there would be greater rigour in their work. A heavily interpretive approach may raise questions about the extent to which the findings are derived from the data (Giorgi, 1992; Giacomini \& Cook, 2000). 
Although description and interpretation are different, they are entangled. Sandelowski (2000) states 'all inquiry entails description, and all description entails interpretation' (p. 335). Descriptions will always depend on perceptions, which are inevitably context-bound. It would be naïve to assume one could describe faithfully and truthfully without interpretation to some degree (Sandelowski, 2000). In qualitative description the researcher does not hope to look with a pure untainted eye but rather seeks to keep inference to a minimum, stay close to the data and present accounts that are simple and likely to result in consensus. Emphasis on description renders qualitative description closer to the positivist end of the spectrum.

The risk with research based primarily on description is that the analysis may be unfocused. At its most basic, a qualitative description could arguably be a total reproduction of the gathered data. This would be unwieldy and clearly of no benefit. In order to reduce the data to something manageable, some analysis and interpretation must take place. Researchers, in interpreting the data, will naturally bring their perspective to bear during this process. Qualitative description does not hope to assume total objectivity in interpreting data, but does exhort the researcher to interrogate the data carefully and critically ensure no statement is made in interpretation that is not explicit in the data.

\subsection{Goodness of $\mathrm{fit}^{2}$}

Any task is more effectively carried out using the appropriate tools and techniques for the job, and research is no different. Morse and Singleton (2001) consider 'fit' an essential element of rigour when collecting and analysing data. Choice of methodology is an important aspect of fit, as the methods chosen must be those able to provide the best answer to the question posed (Morse \& Singleton, 2001; Whittemore, Chase, \& Mandle, 2001). Maternal perception of fetal movements is a subjective phenomenon. A problem of subjective perception demands investigation by qualitative methods. Variation in maternal reporting of

\footnotetext{
${ }^{2}$ The term 'goodness of fit' was used by Lincoln and Guba (1985) in describing 'transferability' or the likelihood that a study's findings 'fit' other contexts (Lincoln \& Guba, 1985). Morse and Singleton (2001) later discussed procedural aspects of 'fit' determining that internal validity commenced with congruence between an emerging research question, its underlying assumptions and the methods and analytic procedures used in exploring that question.
} 
fetal movements has already been established (Heazell et al., 2005; O'Sullivan, Stephen, Martindale, \& Heazell, 2009; Olesen \& Svare, 2004); some women perceive very frequent movements whilst others perceive few or no movements despite actual fetal movements being observed on ultrasound (Gettinger et al., 1978; Hertogs et al., 1979). Qualitative description is a methodology that embraces variation and seeks to provide a broad rather than deep description of qualitative phenomena. For maternal descriptions of fetal movements to be meaningfully drawn on in further work they need to be broad-based and capture both typical and unusual cases. For these reasons qualitative description was felt to be an appropriate methodology for guiding the design and implementation of this study.

\subsection{Study design}

\subsubsection{Participants}

Low-risk women in their first ongoing pregnancy were identified as appropriate subjects for this study. Women in their first pregnancy are thought to pay greater attention to fetal movements and it was felt for this reason they might be more willing participants in a study of fetal movement perceptions. Further, women with more than one child may make comparisons between the current pregnancy and previous pregnancies, which although interesting in itself might have added another element to the data without necessarily furthering the aims. Low-risk women were identified as potential subjects in order to identify a possible baseline for normal fetal activity as perceived qualitatively. For the purposes of this study low risk was defined as a woman without any complications warranting referral to specialist services as per the national referral guidelines (New Zealand. Ministry of Health, 2012). In order to make qualitative analysis of the interview data feasible and yet capture a range of participants likely to be representative, 21 women were recruited. This is a larger sample than might be considered for a qualitative study but was needed in order to encompass expected variation in descriptions. A further aspect of the study was consideration of variations in women's descriptions of fetal activity during pregnancy, and pregnancy outcomes including case characteristics. 


\subsubsection{Inclusion/exclusion criteria}

For this study, 21 low-risk women in their first pregnancy were recruited via five community-based midwifery practices in a provincial city in the North Island of New Zealand. Eligible women had a singleton pregnancy, were under the care of a Lead Maternity Care (LMC) Midwife, and had good spoken English. Women who had conditions warranting transfer of care to a specialist obstetrician at the time of enrolment were not eligible to participate. Two participants interviewed were later excluded as not meeting eligibility criteria (one due to parity, one due to high BMI at booking), leaving the final sample for analysis at 19.

\subsubsection{Interview guide development}

In keeping with the naturalistic approach and minimal researcher inference, questions were broad and open-ended. A pilot interview was conducted with a midwife colleague who was 39 weeks pregnant with her first child (Appendix 1). On feedback from the pilot interviewee it was felt the first question 'Tell me what your baby's movements feel like?' was too broad, and indeed elicited too much disconnected information in one flurry. A better question was proposed, which was 'Do you remember when you first felt your baby move?' and 'What was that like?' This approach proved an excellent introduction and allowed for chronicling of the development of fetal activity over the course of pregnancy by interviewees.

\subsubsection{Ethics}

Ethics approval was obtained from the New Zealand Health and Disability Ethics Committee (Central Region), and locality approval for the various community sites was obtained prior to approaching participants. In preparing for 
ethics application the possibility that discussing fetal movements might cause anxiety in participants was considered, as was the potential for women to reveal concerning information about their baby's movements. In order to address these things it was decided that at the close of the interview women would be given a leaflet about normal fetal movements and, if anything concerning was expressed, they be advised to talk to their LMC. Permission was also obtained from the participants to access pregnancy, labour and birth records, in order to consider birth outcomes in relation to the descriptive accounts.

\subsection{Data collection}

In keeping with the naturalistic approach to data gathering in this study, interviews were undertaken in a setting as close as possible to the normal setting of an antenatal visit, in community midwifery clinics, women's homes and at the hospital. It is usual for midwives to enquire after fetal movements at antenatal visits (Heazell et al., 2008; Smith et al., 2014). This exchange of information about fetal activity constitutes an opportunity for the midwife to provide education for the woman about what to expect in terms of fetal activity, so that the woman may monitor the wellbeing of her own baby and report any concerns, and is also the beginning of fetal movement surveillance as part of antenatal care. The intention was for the interview setting to mimic that clinical moment, with the exception that the woman was invited to provide a more detailed and expanded account of her impressions of her baby's movements than is normally allowed for during an antenatal visit.

\subsubsection{Participant recruitment}

Participants were approached via their LMC midwives. Posters inviting participation in the study were left in midwifery clinic reception rooms and used by LMCs to directly invite women to be part of the study. LMCs had previously been 
given information about the study and asked to consider whether they had suitable low-risk primiparous clients to approach at the time the interviewer was present. LMC practices completed locality-approval documentation as part of ethical approval. Based on the pilot interview, potential participants were advised the interview would take approximately 20 minutes and could be conducted following their antenatal visit that day or arranged to coincide with a later visit or at a time that was convenient to them (see Information Sheet Appendix 2).

\subsubsection{The interviews}

Interviews were conducted at two time points in the third trimester: early (28-32 weeks) and late (37-41 weeks). Consent was signed prior to the first interview and covered all follow-up interviews and collection of data from the pregnancy and birth records (Appendix 3). Questions were both semi-structured and open-ended and primarily asked women to describe how their baby's movements felt, patterns of movements over the day and any factors they noticed that appeared to increase or decrease their baby's movements. An inductive approach to questioning was employed where responses were followed up with clarifying or expanding questions such as 'What do you mean by...?' or 'How did that feel?' in order to gain as detailed descriptions as possible. Interesting or unusual participant responses prompted additional questions to be added to the interview schedule, to be asked of later participants. For example, two participants reported feeling fewer fetal movements during a period of illness. Subsequent participants were then asked if they had experienced any common illness since becoming aware of regular fetal activity and if so had they noticed any change in movements. The majority of later participants had been well throughout pregnancy and no other women reported a reduction in movements. This potential lead did not provide any new or useful information but is an example of the inductive approach taken in this study. 
Early third trimester interviews were conducted face-to-face in the setting of their usual antenatal care, and an audio recording made of the interview for later transcribing and analysis. Late third trimester interviews were conducted as described above, or in some instances by telephone. Early on it was identified that the less said by the researcher the better. A strategy was devised whereby the only researcher responses were in the form of clarifying questions. In this way many of the participant's comments were restated so that there was no confusion about their meaning.

\subsubsection{Birth outcome data}

Participants provided written permission to have their pregnancy/labour and birth record accessed following the birth so that any patterns identified in fetal movement descriptions could be considered alongside outcome data (see Appendix 3). Further permission was gained from the local District Health Board to access patient records for this purpose. A problem identified in this was the incompleteness of the hospital-held record. LMC midwives were requested to provide access to records for copying following interviews but in a number of cases did not respond, meaning analysis of outcome data was limited to data which was complete or relatively complete. Fortunately birth weight, an important retrospective indicator of fetal wellbeing, was available for analysis.

\subsection{Data analysis}

Data analysis was conducted in two modes. The primary mode was qualitative and commenced during data collection. Concurrent collection and analysis of qualitative data allowed for evaluation to be made as to whether the sample size had been sufficient to provide a relatively comprehensive account of fetal activity for low-risk first-time mothers. One aspect of validity in sampling for descriptive data is 'saturation'. Data saturation is considered to be achieved when 
themes and categories become repetitive and redundant, to the extent that no new data is expected to emerge (Polit, 2008). Although saturation was not a stated aim of the study, the final interviews added nothing new, but simply provided further colour in restating the emerging codes. The secondary mode involved considering variation in fetal movement descriptions within the participant group in terms of birth outcome. This aspect of the analysis was largely quantitative and involved use of descriptive statistics to represent variations in maternal accounts of fetal movements reported in this study (eg percentages of women who reported increased as opposed to decreased movements at term). Traditionally, qualitative and quantitative methods have been kept separate (Bazeley, 1999), but in recent decades strong arguments have been made for combining techniques (Morgan, 1998; Morse, 1991; Teddlie \& Tashakkori, 2006). Qualitative description as a methodology is more readily compatible with mixedmethod or combining studies (Sandelowski, 2000). When combining qualitative and quantitative methodologies, one approach generally takes precedence and is complemented by the other (Teddlie \& Tashakkori, 2006). In this study, the qualitative aspect of the enquiry takes precedence and is followed sequentially by the quantitative analysis.

In terms of researcher positioning, the aim is not to explore maternal perception of fetal movements as a social phenomenon, but as a physiological phenomenon with potential to inform further research into stillbirth prevention.

\subsubsection{Qualitative content analysis of interview data}

Interview transcripts were analysed using qualitative content analysis (Hsieh \& Shannon, 2005). As the objective with qualitative description is to provide a rich, straight description, codes were drawn directly from the data so that the descriptions and comparisons might be directly informed by the participant's words rather than by any pre-determined theory or expectation. Organisation of codes into categorical groups then allowed for comparison of the accounts both within subject and between subjects, in addition to consideration of overall themes within maternal accounts of fetal activity. Comparison of accounts within subjects at the 
two time-points led to a chronological account of fetal activity with advancing gestation, whilst comparison between subjects allowed for some consideration of variation.

A master code list was devised of frequently occurring codes. In keeping with the descriptive emphasis of the study, broad-based codes were used (Appendix 4). One-off observations were not included in any analysis. For instance, one woman said that her baby moved more when she was 'happy', the example being when she chatted to her partner on Facebook in the evenings. Although maternal mood may indeed influence fetal behaviour (DiPietro, Hilton, Hawkins, Costigan, \& Pressman, 2002; DiPietro, Irizarry, Costigan, \& Gurewitsch, 2004), every participant reported increased movements in the evening, with only one describing her happiness as a contributory factor. And so, whilst this particular observation was perfectly valid, it was set aside, as inclusion in any final analysis of factors influencing movement perception might be less likely to result in consensus. For this study, descriptions needed to be broad enough to be recognised as valid by a range of pregnant women.

Codes were then grouped loosely into themes, prompting a return to the literature for hypothetical explanations of the oft-repeated observations. Qualitative approaches often borrow from one another and this approach is not dissimilar to data treatment in Glaserian grounded theory, where literature review following data gathering is important for ensuring emerging theories are truly grounded in the data. The process of data analysis according to Glaser is not about turning all of the data into categories and themes, but rather one of loosely generating connections and ideas and explaining them theoretically (Grbich, 2007). Any comparison to grounded theory in this work ends here as there was no intention to press on to a theory of fetal movements, although a number of hypotheses are presented in the discussion section for consideration in further studies. 


\subsubsection{Analysis of outcome data}

Case characteristics and pregnancy-outcome data was analysed according to fetal movement patterns described during pregnancy. Participant numbers were too small to enable analysis of fetal injury or death as these are relatively rare outcomes. However, some limited analysis of fetal movement descriptions according to maternal BMI, and infant characteristics such as birth weight, sex and condition at birth was possible. Outcome data was collected on an Excel spreadsheet, and simple statistics were used to describe, for example, incidence of reporting of various patterns in fetal movement e.g. percentage of women reporting increased strength of fetal movements at term. Birth weight is an important retrospective indicator of fetal wellbeing. Birth weight is influenced by a number of variables, including maternal height and weight, gestation and sex of the baby. Therefore birth weights were converted to a customised birth weight centile (CBWC) using GROW (gestation-related optimal weight) to control for these variables. Variations in fetal movement patterns were considered in terms of CBWC in a number of instances. 


\section{Chapter 4 Findings}

\subsection{Introduction}

During data collection and analysis a number of strong themes emerged. These are presented in this chapter as three sections: the first is a chronological account of fetal movement quality as perceived by participants, entitled 'Maternal perception of fetal movements with advancing gestation'; the second explores a novel finding of 'Fetal responses to maternal hunger and satiation'; and the third section addresses three key aspects of fetal movement patterns as described by women in the study entitled 'Temporal, positional and kinaesthetic aspects'.

Participant quotes have been used to exemplify statements informing the themes. Participants were assured anonymity and therefore pseudonyms have been used with these quotes. Where there is variation in maternal accounts and significant minority themes emerge, some descriptive statistics are used to quantify the variation and describe associated case characteristics.

\subsection{Section One: Maternal perception of fetal movements with advancing gestation}

Fetal activity at term is poorly defined and whether or not movements are reduced is a matter of controversy. One of the aims of this study was to explore accounts of fetal activity over the third trimester in order to better understand any changes that may occur. A chronological account of fetal movements emerged from the data as participants described their baby's movements, recalling how they had changed from earlier in the pregnancy.

\subsubsection{Quickening}


Participants were asked to recall when they first felt their baby ${ }^{3}$ move, and to describe the sensations as if to a close friend or family member. The purpose of these questions was to put participants in touch with their initial sensory memory of fetal movements and to elicit plain language descriptions of the sensations.

Participants had often read about or heard from others what the first movements felt like and keenly anticipated experiencing quickening for themselves. Feelings of uncertainty often accompanied the first time a mother noticed fetal movements, leading women to question whether it really was a fetal movement they had felt or some other bodily sensation. Fetal movement was suspected when the sensation was identified as something new, something they hadn't felt before. With increasing repetition of the sensations women could confidently say they had felt their baby move.

'It was just one little tiny movement and I wasn't sure if it was, but then movements after that felt the same.' Gabrielle

The absolute smallness and lightness of the movements was emphasised by women when using words like 'knock', or 'flick' to describe them. Some participants, in order to illustrate how small the movements were, even forwent words and instead uttered small sounds such as 'dink' or 'ugh' or used hand gestures such as a holding an imagined tiny object in a pincer grip. Fetal movements were identified by some as 'small bumps' felt when placing hands on the belly, but for most the sensations were primarily internal.

First fetal movement descriptors were often watery in nature. The movements might feel 'floaty' or 'fluttery', like 'bubbles moving below the surface' or like a 'worm swimming in the belly'. Some described feeling the baby's contact with the wall of the uterus and its impulse transmitted through the abdominal wall.

'Imagine yourself swimming in a pool, and then hitting a glass on the outside or something, and then try to imagine that inside your belly.' Anahera

\footnotetext{
${ }^{3}$ When addressing women directly about fetal movements the term 'baby' is used. The term fetus is retained in other parts of the thesis as it refers specifically to the baby that is in the uterus, with all the physiological contingents that implies: sharing the mother's bodily environment, floating in amniotic fluid and dependent on the placenta.
} 
Identification of fetal movements made the pregnancy real. Some used the word 'weird' to describe first fetal movements, but were quick to elaborate they weren't weird in an unpleasant way but in the sense of being a strange, new sensation providing a profound indication of impending motherhood. For one young participant, quickening brought home the reality of the pregnancy and also sparked feelings of happiness.

'It was unusual for me at first 'cause I was still getting it into my head, "I'm pregnant, I'm pregnant." It was a real shock but I kind of felt happy at the same time too, yeah.' Beth

Movements after quickening were sporadic in frequency, and some women described long periods of not feeling movements following their initial identification. As the pregnancy progressed, increasing strength and frequency of movements made them easier for the woman to identify. The regular reminder of the baby's presence through movement gave some participants a sense of being accompanied. Ongoing perception of fetal movements was regarded as an enjoyable aspect of pregnancy that was reassuring of normal pregnancy progress and the baby to come.

I like it because it lets me know that the baby is there. If it's moving it's got to be a good thing. I didn't have a 12 week scan, so that was the first sign, apart from getting slightly fat,' (laughs) 'that there was something growing in there. So that's why I stop and notice them [the movements] during the day because I sort of enjoy them as they come along. That's my way of bonding I guess, because I don't know what I am having. So, I feel connected.' Jane

\subsubsection{Fetal activity at 28-32 weeks}

With advancing gestation, participants described a sustained increase in strength, frequency and, most notably, variation in fetal movements. Strength of movements increased so that there was now no doubt that the sensations were fetal movements. Where movement sensations at quickening had been primarily 
internal, by 28 to 32 weeks participants reported that they could now see the fetal parts moving under the skin and feel many movements with their hands on the abdomen.

'I have only just started to notice them on my skin. Like before that they felt like they were quite a way inside, like you can sometimes see the actual skin moving. I can't tell what it is; like an elbow, knee or foot, but just seeing the skin move.' Jane

One exception to this was a participant with an anterior placenta who reported that movements were all internal and she was unable to feel them with her hands. Frequency increased to the extent that how often the baby moved was more likely to be defined according to length of time without movements, as opposed to number of movements felt.

'Probably four weeks ago like I was getting maybe six to seven kind of [approximate] movements. And then since then it's probably like every, at least every half an hour to three quarters of an hour, I feel something.' Louise

Whilst fetal movements had become increasingly apparent by $28-32$ weeks, frequency varied considerably between participants. Some women reported feeling fetal activity 'all the time', whilst for others movements were largely absent during the day and only noticeable in the evening. Occurrences of fetal movements or fetal quiescence were closely tied to environmental factors such as time of day, maternal position and maternal activity and rest. Women reported increased fetal activity later in the day and most especially on going to bed. Sitting or lying positions were reported to promote fetal activity, as did maternal hunger or eating. Fetal quiescence was more likely to occur during the daytime, when the woman was ambulant, and in some cases following meals.

In addition to these environmental factors a number of stimuli were reported to prompt fetal movements, including: loud or unexpected sounds (including voices); pressure on the abdomen either from a cramped sitting position; unintentional bumping of the woman's abdomen (by young children for example); palpation by the midwife; or gentle pushing of fetal parts by the mother. 
The most prominent feature of maternally reported fetal activity at 28-32 weeks was its great variation in both strength and types of movement. Women described a considerable repertoire of fetal actions at this gestation. Smaller softer actions included: 'wriggling', 'pressing', 'tickling', 'playing with hands', 'snuggling', 'massaging' and 'soft tapping'. Forceful and vigorous actions included: 'rolling', 'kicking', 'flip-flopping', 'tumbling',' turning', 'swooping', 'pumping' and 'somersaulting'. Women described large, complex movements which might involve movement within many areas of the abdomen, often in a sequential moving of parts, creating a ripple pattern. These movements were interpreted by some mothers as stretching or dancing.

'So you know sometimes it's just lovely little taps, but then other times it's quite strong. And the stomach will actually move with the stronger movements, either in wave or the whole stomach will jolt - depending. And sometimes she does like a dance, 'cause it's sort of, you know, it's not just the one point of contact, there's lots, all over the stomach.' Roimata

The variation in movements was often described as 'random', and the strength at times surprising, almost alarming. Strange movements were also recounted, such as startles, jumping movements 'as if they have got a fright', or spasm-type movements. 'Jerky' and 'jolty' were frequently-used descriptors for movements at this gestation. Movements might be so strong and sudden at times that the woman could feel herself thrown off balance or winded by the sudden lurch in the belly.

I like the little ones and I don't like feeling like my whole body is going to give way. When the baby ... I don't know what it's doing, whether it's turning right round, or pushing on something and making me lose my balance.' Jane

Some expressed excited trepidation at the prospect of the baby continuing to grow in size and strength, wondering should the vigour continue to increase at this rate, how they might tolerate the movements at term. 


\subsubsection{Fetal activity at term}

At term, the impressive variation in movements reported earlier had subsided. 'Jerky', 'jolty' or 'startled' movements disappeared or were rarely reported. Movements were described as 'all the same' or 'consistent' as opposed to random and unpredictable as they had been earlier in the pregnancy.

'It's like baby knows what they are doing now so they do it all the time.' Anahera

There was still variation in movement-types such as whole-body twisting and rolling, kicking, and lighter tickling hand movements felt in the pelvis, but the overall variation was more constrained. Fetal hiccups were more commonly reported by participants at term as compared to earlier. However, some participants reflected that they had probably felt hiccups earlier but only recently identified what the sensation was.

Dominant movement types were described as pushing, rolling and stretching, but most especially pushing. Some participants re-enacted this pushing action by flexing and then extending both their arms as if to demonstrate a bilateral fetal leg extension. When the fetus pushed in this way the bottom would move up and out from the mother's body and then settle back again. All women in this sample had a cephalic presentation at term. The change from frequent kicking movements to strong pushing movements was commonly described as below.

'Oh heaps more stronger, but it's not so much kicking, it's pushing, it's pushing now, pushes out and around and yeah... There's not the constant kicking feeling. It's like waves kind of in your stomach, like pushing out and moving it along.' Tahlia

Where increased variation had been the dominant feature of fetal movements at 28-32 weeks, increased strength was the most notable feature at term. Maternal descriptions of types of fetal movements, and the change from jerky and random movements in the early third trimester to sustained pushing and rolling movements at term, was recounted with remarkable consistency across the sample. Descriptions of changes in frequency, however, were not consistent. For the 18 women who were interviewed at term, frequency of movements was either 
unchanged $(44.4 \%, n=8)$ or reduced $(38.9 \%, n=7)$, and increased in a minority of participants $(16.7 \%, \mathrm{n}=3)$, when compared to earlier (Table 1). Strength of movements were increased in the majority of participants $(50 \%, n=9)$, unchanged in a third (33\%, $n=6)$, and decreased in just three (16.7\%) (Table 1).

Table 1. Distribution of changes in strength and frequency of fetal movements at term

\begin{tabular}{|l|l|}
\hline Strength of movements at term & Frequency of movements at term \\
\hline Increased (9) $50.0 \%$ & Increased (3) $16.7 \%$ \\
Unchanged (6) $33.0 \%$ & Unchanged (8) $44.4 \%$ \\
Decreased (3) $16.7 \%$ & Decreased (7) $38.9 \%$ \\
\hline
\end{tabular}

Participants were not asked directly if movements were increased or decreased in terms of frequency or strength. These determinations were made following analysis of interview responses to the question, 'Tell me what your baby's movements are like now that you are almost due.'

Because there was considerable variation in descriptions of strength and frequency at term, further analysis was undertaken to determine whether case characteristics had any bearing on differences in strength and frequency of movements at term. Some interactions were noted between these parameters and maternal body mass index (BMI), birthweight, and fetal indications for delivery. Participant characteristics are represented in Table 2.

\subsubsection{Variation in strength and frequency of fetal movements at term in relation to birthweight.}

In this study maternal reports of increased strength and increased frequency of movements at term were associated with higher mean customised birthweight centile scores. For women who reported increased strength of movements at term, mean CBWC of newborns lay on the $46.8^{\text {th }}$ centile, as compared to the sample, mean CBWC of 39.6. For women who reported frequency was increased, mean CBWC was 53.4 as compared to the overall mean CBWC of 39.6. In this study, no woman who described increased strength 
or frequency of fetal movements at term delivered a baby with a CBWC under the $25^{\text {th }}$ centile. Conversely, decreased strength and decreased frequency were associated with lower mean CBWC scores. Those women who reported decreased strength of movements delivered infants with a mean CBWC of 27, as compared to 39.6, whilst those who reported decreased frequency delivered infants with a mean CBWC of 31, compared to 39.6. (Table 3). Therefore there was a tendency to increased birthweight of infants where women had described increased strength and or frequency of movements at term, and smaller infant size where movements were described as reduced in either frequency or strength. 
Table 2. Participant characteristics

\begin{tabular}{|c|c|c|c|c|c|c|c|c|c|c|c|}
\hline Mother & Age & Employment & $\begin{array}{l}\text { Marital } \\
\text { status }\end{array}$ & BMI & $\begin{array}{l}\text { Placental } \\
\text { location }\end{array}$ & Gest & Sex & Birthweight & $\begin{array}{l}\text { Customised } \\
\text { birthweight } \\
\text { centile }\end{array}$ & $\begin{array}{l}\text { Mode of } \\
\text { delivery }\end{array}$ & Abnormal delivery indication \\
\hline 1 & 28 & EMPLOYED & MARRIED & 23 & anterior & 37 & $\mathrm{M}$ & 3375 & 81 & Ventouse & Prolonged 2nd stage \\
\hline 2 & 26 & EMPLOYED & MARRIED & 23 & posterior & & $\bar{F}$ & 3010 & 13 & $\overline{\text { NVB }}$ & NONE \\
\hline 3 & 28 & EMPLOYED & MARRIED & 26 & anterior & 40 & $\mathrm{M}$ & 3580 & 86 & NVB & NONE \\
\hline 4 & 28 & EMPLOYED & SINGLE & 26 & posterior & 39 & $\mathrm{~F}$ & 3060 & 13 & NVB & NONE \\
\hline 5 & 33 & EMPLOYED & DEFACTO & 33 & anterior & 41 & $\bar{M}$ & 4130 & 50 & Em LUSCS & Fetal bradycardia \\
\hline 6 & 19 & UNEMPLOYED & $\overline{\text { SINGLE }}$ & 25 & posterior & $\overline{41}$ & $\bar{M}$ & 4040 & 60 & Forceps & fetal distress \\
\hline 7 & 27 & UNEMPLOYED & SINGLE & 23 & $\begin{array}{c}\text { posterior/ } \\
\text { fundal }\end{array}$ & 40 & $\mathrm{~F}$ & 3600 & 74 & NVB & $\overline{N A}$ \\
\hline 8 & 26 & EMPLOYED & DEFACTO & 23 & posterior & $\overline{41}$ & $\bar{M}$ & 3645 & 33 & Em LUSCS & Obstruction CPD \\
\hline 9 & 30 & EMPLOYED & MARRIED & 22 & $\begin{array}{l}\text { posterior/ } \\
\text { fundal }\end{array}$ & 39 & $\bar{M}$ & 3865 & 74 & $\overline{\text { NVB }}$ & $\overline{\mathrm{NA}}$ \\
\hline 10 & 20 & UNEMPLOYED & DEFACTO & 21 & & 40 & $\mathrm{M}$ & 3510 & 41 & NVB & $\overline{\mathrm{NA}}$ \\
\hline 11 & 34 & EMPLOYED & MARRIED & 23 & No data & 41 & $\mathrm{~F}$ & 3215 & 18 & Ventouse & $\begin{array}{l}\text { POOR PROGRESS ACTIVE } \\
\text { PHASE }\end{array}$ \\
\hline 12 & 29 & EMPLOYED & MARRIED & 19 & posterior & 37 & $\mathrm{~F}$ & 3115 & 36 & NVB & $\overline{N A}$ \\
\hline 13 & 29 & EMPLOYED & MARRIED & 28 & posterior & 40 & $\mathrm{~F}$ & 2990 & 3 & FORCEPS & Ventouse extraction failed \\
\hline 14 & 27 & EMPLOYED & MARRIED & 23 & anterior & 39 & $\bar{F}$ & 2930 & 17 & $\overline{\text { NVB }}$ & $\overline{\mathrm{NA}}$ \\
\hline 15 & 20 & STUDENT & MARRIED & 32 & posterior & 40 & $\mathrm{M}$ & 3790 & 48 & NVB & $\overline{N A}$ \\
\hline 16 & 22 & EMPLOYED & DEFACTO & $\overline{34}$ & posterior & 40 & $\bar{F}$ & 3465 & 26 & $\overline{\text { NVB }}$ & $\overline{\mathrm{NA}}$ \\
\hline 17 & 27 & UNEMPLOYED & MARRIED & 23 & posterior & 41 & $\mathrm{~F}$ & 2990 & 4 & Em LUSCS & Fetal distress \\
\hline 18 & 32 & EMPLOYED & MARRIED & 29 & posterior & $\overline{41}$ & $\bar{F}$ & 3590 & 34 & $\overline{N V B}$ & $\overline{N A}$ \\
\hline 19 & 19 & UNEMPLOYED & SINGLE & 25 & anterior & 41 & $\bar{M}$ & 3655 & 42 & Em LUSCS & $\begin{array}{c}\text { POOR PROGRESS ACTIVE } \\
\text { PHASE }\end{array}$ \\
\hline
\end{tabular}


Table 3. Changes in strength and frequency of fetal movements at term in relation to customised birthweight centile

\begin{tabular}{|c|c|c|c|c|c|c|c|}
\hline \multirow[t]{2}{*}{ Participant } & \multirow{2}{*}{$\begin{array}{c}\text { Birthweight } \\
\text { Centile }\end{array}$} & \multicolumn{3}{|c|}{ Strength of Fetal movements } & \multicolumn{3}{|c|}{ Frequency of Fetal Movements } \\
\hline & & Increase & no change & decrease & increase & no change & decrease \\
\hline 3 & 86 & $x$ & & & $\mathrm{x}$ & & \\
\hline 1 & 81 & - & - & - & - & - & - \\
\hline 7 & 74 & $\bar{x}$ & & & & $\bar{x}$ & \\
\hline 9 & 74 & & $x$ & & & $x$ & \\
\hline 6 & 60 & & & $\bar{x}$ & & & $\bar{x}$ \\
\hline 5 & 50 & $x$ & & & & & $\bar{x}$ \\
\hline 15 & $\overline{48}$ & & $x$ & & & & $\bar{x}$ \\
\hline 19 & 42 & $\bar{x}$ & & & $\bar{x}$ & & \\
\hline 10 & 41 & $\bar{x}$ & & & & $\bar{x}$ & \\
\hline 12 & 36 & $x$ & & & & $\bar{x}$ & \\
\hline 18 & 34 & $\bar{x}$ & & & & $\bar{x}$ & \\
\hline 8 & 33 & $x$ & & & $\bar{x}$ & & \\
\hline 16 & 26 & $x$ & & & & & $x$ \\
\hline 11 & 18 & & & $x$ & & & $x$ \\
\hline 14 & 17 & & $\bar{x}$ & & & $\bar{x}$ & \\
\hline 2 & 13 & & $x$ & & & $\bar{x}$ & \\
\hline 4 & 13 & & & $x$ & & & $x$ \\
\hline 17 & 4 & & $x$ & & & & $x$ \\
\hline 13 & 3 & & $x$ & & & $\bar{x}$ & \\
\hline $\begin{array}{l}\text { Mean } \\
\text { customised } \\
\text { birth- } \\
\text { weight } \\
\text { centile }\end{array}$ & 39.6 & 46.8 & 26.5 & 27 & 53.6 & 36.5 & 31.3 \\
\hline
\end{tabular}

\subsubsection{Body Mass Index}

Participant BMI $\left(\mathrm{kg} / \mathrm{m}^{2}\right)$ ranged from 19 to 33 , with three women meeting the definition of being obese i.e. BMI more than 30 (women with a BMI of more than 35 were excluded from this study). All participants in this study who were clinically obese (n 3 ) reported a relative reduction in frequency of fetal movements at term.

\subsubsection{Operative delivery}


In total, eight participants required operative delivery, defined as caesarean section, ventouse or forceps delivery. Data on fetal movements at term were available for seven of these (one delivered at 37 weeks before a term interview could be undertaken). No relationship was noted between mode of delivery and quality of fetal movements at term with the exception of all three cases where surgical delivery occurred for a primarily fetal indication (eg fetal distress, fetal bradycardia), all of which fell into the reduced frequency of movements at term category.

Table 4. Participant characteristics and changes in strength or frequency of fetal movements at term

\begin{tabular}{|c|c|c|c|c|c|c|}
\hline \multirow[t]{2}{*}{$\begin{array}{l}\text { Case Characteristic (total } \\
\text { number) }\end{array}$} & \multicolumn{3}{|c|}{ Strength of movements at term } & \multicolumn{3}{|c|}{$\begin{array}{l}\text { Frequency of fetal movements } \\
\text { at term }\end{array}$} \\
\hline & $\begin{array}{l}\text { increase } \\
\text { (9) }\end{array}$ & $\begin{array}{l}\text { no change } \\
\text { (6) }\end{array}$ & $\begin{array}{l}\text { decrease } \\
\text { (3) }\end{array}$ & $\begin{array}{l}\text { increase } \\
\text { (3) }\end{array}$ & $\begin{array}{l}\text { No } \\
\text { change } \\
(8)\end{array}$ & $\begin{array}{l}\text { decrease } \\
(7)\end{array}$ \\
\hline Maternal obesity (3) & 1 & 1 & 1 & & & 3 \\
\hline Birthweight below $10^{\text {th }}$ centile (2) & & 2 & & & 1 & 1 \\
\hline Birthweight below $20^{\text {th }}$ centile (6) & & 4 & 2 & & 3 & 3 \\
\hline Emergency caesarean section (4) & 3 & 1 & & 2 & & 2 \\
\hline $\begin{array}{l}\text { Instrumental birth (forceps or } \\
\text { ventouse) (4) }\end{array}$ & & 1 & 3 & & 1 & 3 \\
\hline $\begin{array}{l}\text { Operative delivery for fetal } \\
\text { indication (3) }\end{array}$ & 1 & 1 & 1 & & & 3 \\
\hline
\end{tabular}

Of the seven cases where fetal movements were reported as being reduced in frequency at term, all were associated with either maternal obesity, birthweight under the $20^{\text {th }}$ centile or operative delivery for fetal indications, with some falling into more than one of these categories. No relationship was noted between fetal movement quality at term and fetal sex, maternal age or placental location. 


\subsubsection{Maternal monitoring of fetal activity}

It is important to note that for this study reduced frequency of movements was determined from analysis of transcripts and not from clinical presentations for reduced fetal movements. The majority of women in this sample reported feeling concerned about fetal movements at some stage during the pregnancy $(78.9 \%$ 15/19). Women considered contacting their midwife when they noted a period of reduced movements, but very often took measures to encourage their baby to move before doing so. These self-assessment measures generally constituted repeating actions that had previously been noted to prompt movements or noting whether the baby responded spontaneously to a stimulus as they normally would. Examples of actions that prompted movement included: rubbing or prodding the belly, taking a warm bath, bringing up the legs to compress the abdomen, or simply sitting or lying down to await movements following a period of being upright and moving about. Where the fetus responded spontaneously to a recognised stimulus this was felt to be reassuring. Some joked that movements in response to their own testing were so vigorous as to make a mockery of their earlier concern.

\subsubsection{Summary}

Types of fetal movements as described by participants changed as pregnancy advanced so that movements in the early third trimester were characterised by their great variation and included random and jerky movements. By term the variation in movement types was more constrained, jerky movements had subsided and pushing or stretching movements dominated. In this study a broad range of properties were accounted for in participant descriptions of fetal movements including: numerous fetal actions; variation in strength; responses to maternal activity; and position changes, diurnal variation and external stimuli. These variations came to be an expected part of fetal activity for the pregnant women and were spontaneously monitored by participants in self-devised qualitative fetal movement assessment. 


\subsection{Section 2: Fetal response to maternal hunger and satiation}

A novel and unexpected finding emerging from this study was a complex fetal response to maternal hunger, eating and satiation. During interviews, participants were asked whether there was a pattern to their baby's movements and if so, to describe it. Mealtimes were identified by the majority of participants $(73.6 \%, 14 / 19)$ as times when fetal activity was likely to be increased in some cases (5) no further detail was offered and the reference was coincident with sitting position. In eight cases however participants explicitly recounted increased fetal movements in response to hunger or eating. Of these eight, seven women (36.8\% 7/19) described increased fetal activity in association with maternal hunger or the period prior to meals. Following meals five of the women who had described increased movement with hunger (26.3\%) described a notable period of quieting which they interpreted as their baby being sated and content. Whilst for 3 women the baby was active after the meal, in two such cases the movement represented continuation of the increased activity noted with hunger. Just one woman described increased fetal activity after a meal, without having first described increased movements with hunger. Thus where fetal activity in relation to meals was described in detail by participants in this study, the predominant pattern was one of increased fetal activity prior to meals and decreased fetal activity following meals with a transition period during which some fetuses might continue their increased activity during and immediately after the meal and others might settle quickly into a quiescent period.

In describing changes in fetal activity around mealtimes participants commonly 'voiced' their baby in an attempt to convey the 'attitude' expressed by the fetal movements. The character of the increased activity prior to a meal was interpreted by the pregnant women as a fetal expression of anticipation of a meal, giving way to frustration when the meal was delayed. Whilst, the nature of the movements perceived following a meal were interpreted as indicating happiness or contentment. In this way fetal movements appeared to function as a form of communication between mother and baby, effectively prompting the mother to eat when she had gone for longer than usual without a meal and then indicating to the mother when the fetus was sated. 
These changes in activity were outlined by participants primarily in response to the question 'Is there anything that makes your baby move more or less than usual?' A typical example of increased fetal movement before meals is as follows:

'Sometimes if I haven't eaten for a while. It could just be me [pause], but you just feel that sometimes she'll make her presence known and go "I need some food here" or something, well that's what it feels like. And until you have something to eat and then she'll settle down again.' (Bridget, 37 weeks)

Those participants, who reported increased activity in association with both hunger and the period following eating, described the movements as changing qualitatively during the course of meal, as in the following example:

So if I'm either hungry... I notice if I haven't eaten, like that's what happened this afternoon, she was not impressed. And she just gets really wriggly and really squirmy. And then after I've eaten, you know, so similar, although it doesn't [pauses] it feels a lot more comfortable after l've eaten, but she goes through a similar thing where she gets quite active.' (Roimata, 39 weeks).

However for five of the seven women who had described increased fetal movement with hunger, the post-prandial period was associated with a marked quieting of fetal activity.

'It seems to happen several times a day; like I suppose it is more around meal times, like before breakfast, she starts getting a bit excited, but yeah, afternoon tea time; any of those times as well, she's still, she goes: "Oh yeah, food's on the way, yay." ... after a meal she's completely silent... usually for, even up to an hour she'll not, like I won't feel a single movement 'cause it's almost just like she's just chowing down and happy.' (Ruth, 39 weeks)

Increasing fetal activity in advance of meals was further intensified where a meal was delayed, as in the example below: 
'She gets very excited just before dinner time, like just before any meal time; and it's not even when I'm cooking. Sometimes I can just walk past the room that has food in it and if I'm not eating within 20 minutes then she starts getting quite irate! Like I must have somehow triggered that I was out to eat and then when I didn't she was hugely disappointed.' (Ruth, 39 weeks)

Fetal activity as described by women in relation to mealtimes demonstrated a developmental pattern over the course of a mealtime period, with staged responses indicative of anticipation, hunger, appreciation of food and satiation, as represented in Figure 1
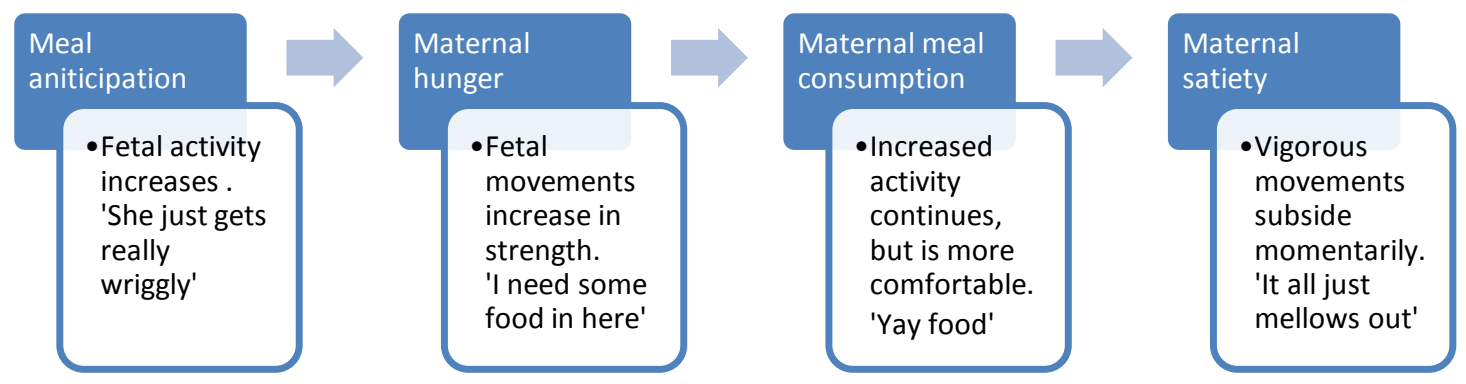

Figure 1. Staged fetal responses to hunger and satiation as described by participants

In this study, just seven of the 19 participants reported increased activity with maternal hunger, whilst 12 did not. When a theme is identified in a qualitative study it is important to consider the negative cases. In this study, just seven of the 19 participants reported increased activity with maternal hunger, whilst 12 did not. When participants who reported increased fetal activity in association with maternal hunger were compared to those who did not it was noted that those women in the study who had provided the most complex and detailed descriptions of their baby's movements were more likely to describe their babies as 'very active' or as 'moving all the time' recounted increased fetal activity with hunger. 
Some analysis of other factors such as maternal BMI at booking, fetal sex and birth outcome was also conducted but is not reported here as no significant differences were identified in this small sample. It was noted however that the babies born to mothers who described increased fetal movements with hunger were smaller than those that did not describe this pattern $(3221.75 \mathrm{~g}$ vs. $3583.75 \mathrm{~g})$ with a mean birthweight difference between groups of 364gm. This difference was compared using a two tailed t test and did reach statistical significance $(\mathrm{t}(17)=-$ 2.295, $p=0.035)$.

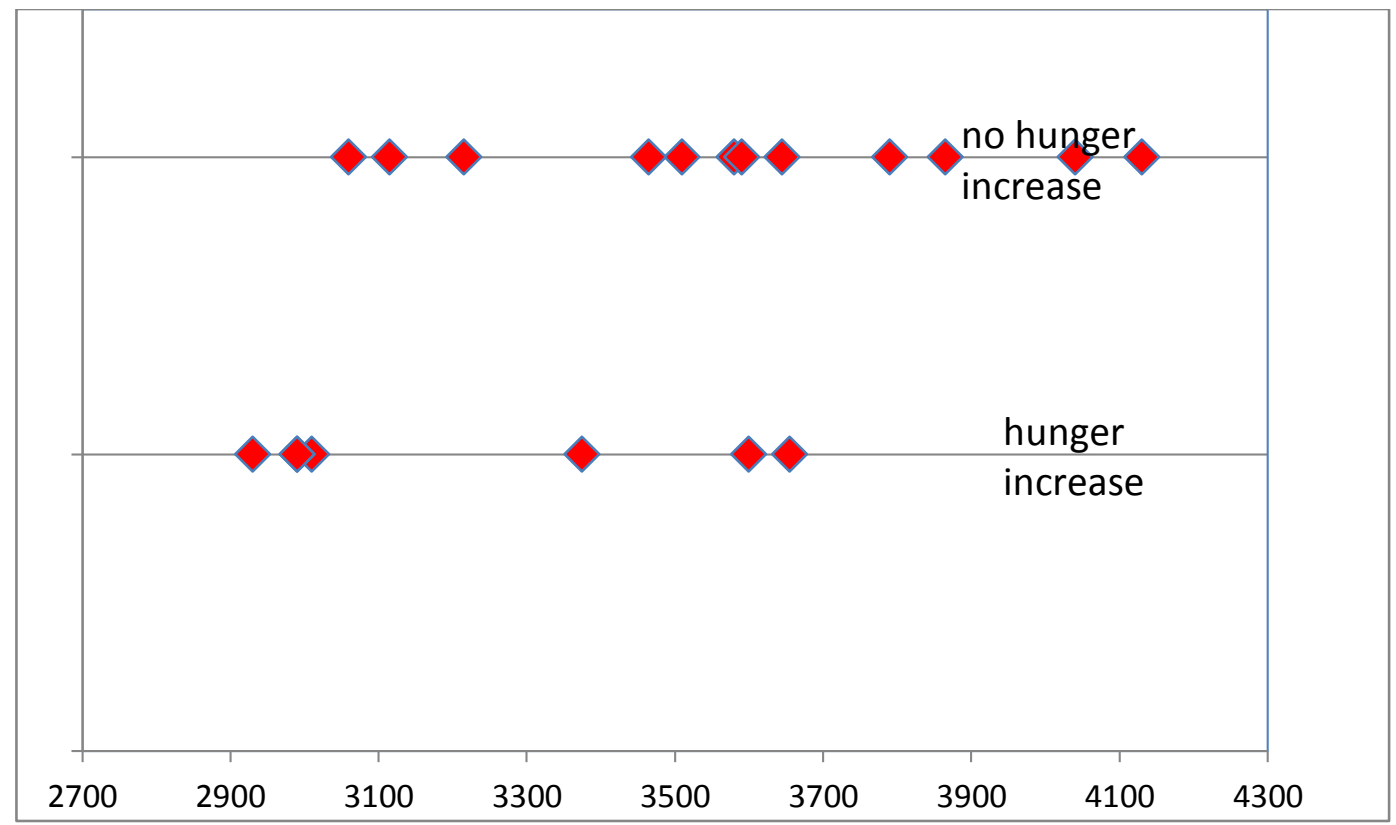

Figure 2 Maternal reports of increased fetal activity in the context of maternal hunger and subsequent birthweight.

Scatterplot of birthweight in grams of babies whose mothers reported increased fetal movement in the context of hunger (bottom line) and those who did not report increased fetal movement in the context of hunger (top line). Nb There are two babies of the same weight $(2990 \mathrm{gm})$ in the hunger series.

When infant birth weights are represented on a scatterplot as shown in Figure 2, it can be seen that there is a preponderance of smaller babies in the hunger group. When birthweights were converted to customised centile scores using the GROW programme (Gestation Related Optimal Weight), which adjusts for maternal height, weight, parity and ethnicity, as well as the sex and gestational age of the baby (Gardosi \& Francis, 2009) a similar distribution can be seen (figure 3). Birthweight has a normal distribution, with the majority of newborns in any given sample expected to fall between the $25^{\text {th }}$ and $75^{\text {th }}$ centiles (Wilcox, 
2001). As can be seen in figure 3 , of the 19 babies in this study, two were born under the $5^{\text {th }}$ centile, both in the hunger group, and six were born under the $20^{\text {th }}$ centile, four of which were in the hunger group.

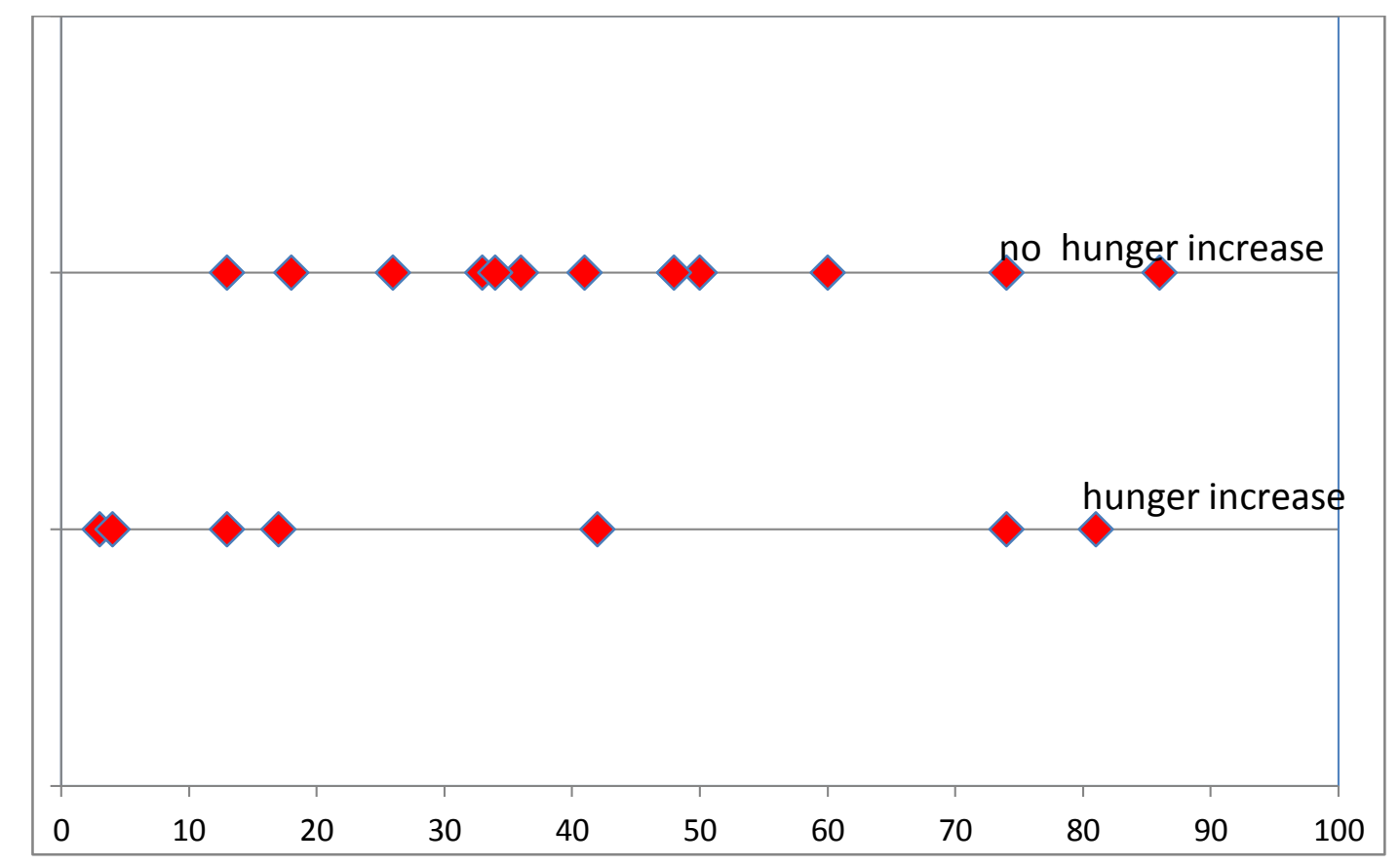

Figure 3 Maternal reports of increased fetal activity in the context of maternal hunger and subsequent birthweight expressed as a customised centile.

Scatterplot of customised birthweight centile of babies whose mothers reported increased fetal movements in the context of hunger (bottom line) compared to those who did not (top line).

\subsubsection{Summary}

In this study, increased fetal activity was commonly reported by participants to be associated with mealtimes. Increased activity in advance of meals in particular was regarded by some participants as a fetal demand for food, often prompting the mother to eat. Fetal activity in association with maternal hunger in this study was preferentially reported by women who later delivered small infants, suggesting these movements are a compensatory response to low fuel supply. In the quest to better understand normal and pathologic fetal activity, this study raises more questions than answers. 


\subsection{Section Three: Maternal perception of fetal movements: temporal, positional and kinaesthetic elements}

Aspects of fetal activity other than frequency may be important for understanding normal and pathologic fetal movements. Within participant descriptions, fetal movement, time of day, maternal position and maternal movement were commonly described contributory factors in fetal movement patterns.

\subsubsection{Temporal aspects}

Participants were asked if their baby had a pattern to their movements and if so to describe it. Participant descriptions of fetal movements in this study were highly consistent with a fetal circadian rhythm. There was considerable variation between participants in reported frequency of fetal movements, yet patterns of movement in terms of times of day when fetal movements were more likely to be perceived were described with relative homogeneity. A typical pattern of movements over the course of the day involved some movement in the early morning, often coinciding with the mother waking, relative quiescence over the remainder of the morning, increased likelihood of movements in the afternoon, especially lunchtime or afternoon tea time, and for all participants a marked increase in movements in the evening with the most fetal activity occurring on going to bed for the night. A typical description of daytime activity is as follows:

'So, like first thing in the morning there'll be like one or two movements that I'll pick up on and they're normally really small; they're definitely not a boot or anything like that, just kind of almost a squiggle for want of a better term, which is cool. It's like yeah, okay, sweet, we'll rock into the day. And then it's not until really mid-afternoon, if it's a normal day, yeah baby kind of gets up to play as such and makes their presence well known.' Serena

'She'll be more active at different times of the day but the most active times are usually afternoon tea time she seems to be probably the most active and 
sometimes if l've been quite active just before bed, as soon as I try and settle down it's like she's going crazy. Eventually she settles down but it takes a while.' Ruth

Participants were also asked if they were aware of fetal movements during the night. All women reported frequent movements on going to bed, which were troublesome for some trying to go to sleep. Aside from the period when they were first trying to go to sleep, participants reported they were not disturbed by fetal movements during the night. All reported waking during the night, but in only two cases was fetal activity reported to contribute to waking. Most reported very little or no fetal movements at these times and a number of participants felt that their baby remained asleep through the night-time toilet excursions.

Maternal descriptions of patterns of fetal activity and rest over the waking hours invariably included aspects of maternal position, ambulation and rest, as in the quote above. Each of these aspects demands consideration in turn.

\subsubsection{Positional aspects}

Maternal position influenced fetal movements according to many participants. Movements were reported to be more intense when lying down, reasonably intense when sitting, and lighter when standing. Many felt that the fetus had more room to move when they were lying down.

'And then yeah when I do go to sleep I'm obviously very relaxed when I'm going to bed and I do feel like my body is more open when I'm lying down; so I do think it is more comfortable for her and I think she just sort of knows that I'm not doing anything else and it's sort of her time to play.' Roimata

Maternal changes of position were also reported to influence fetal activity. Some women described fetal activity increasing when they adopted a position less favoured by the fetus. In these situations the movements were so vigorous as to be interpreted by the woman as a protest. Examples of this included the woman sitting in a cramped position (such as in the car) or lying on a side less preferred by the fetus. Participants would commonly voice their baby in recounting these 
strong kicking movements, eg ('Oh, stop squashing me; get off.' Beth). Participants were often prompted to shift their weight or change their position entirely in response to these types of movements.

This maternal-fetal interaction in regard to position was also described in the reverse, where the fetus is prompted to change its position by a maternal position change, commonly described by participants as something that occurred when they rolled from one side to the other during the night, and was interpreted as the fetus adjusting to the new position and getting comfortable.

'So, probably more thinking like when I go to bed. If I am lying on my side and then I roll over to the other side, it is almost like it readjusts. Like wriggles around and then stops.' Gabrielle

\subsubsection{Kinaesthetic aspects}

According to participants in this study, the most significant influence on perception of fetal activity over the course of the day was the woman's own activity and rest. Participants described an inverse relationship between their own body movement and that of the fetus. Periods of maternal activity were associated with fetal rest, whilst the maternal change from active to quiet seemed to prompt an opposite change in mode from quiet to active on the part of the fetus.

The majority of women in this study were in paid employment during their pregnancy and many described not being aware of movements during the work day, especially when they were ambulant. Participants whose daily routine involved periods of inactivity in the morning, such as sitting during work meetings, or primarily sedentary work, reported strong fetal movements at these times. Thus, although morning quietude was commonly reported, there were exceptions when the mother was relatively still.

Some pondered whether the perceived decrease in fetal activity during ambulation was because they themselves were preoccupied by other tasks. However, many reported explicitly that their own activity seemed have a soporific effect on the fetus, whilst their stillness seemed to promote fetal movement. 
'I think that when I'm moving around maybe the baby tends to go to sleep, 'cause it's not like a rocking motion but because you're moving it must like, make it sleepy; well that's what I think.' Louise

Sitting and relaxing on the other hand seemed to be an invitation for increased fetal activity. Some described their baby as being bored when they were not moving and needing to make their own fun by moving about when the mother rested.

If I'm up walking around then I go and sit down, I've woken him kind of, I feel, and then he starts to squirm around in there.' Tahlia

Participants in this study came to expect bouts of fetal activity when they sat and rested and were reassured by this familiar response as pregnancy progressed. An exception to this was where the mother had engaged in more vigorous activity such as moderate exercise or a strenuous walk in which case the transition from quiet to active on the part of the fetus was reported to take longer.

\subsubsection{Summary}

Fetal activity is reassuring to pregnant women who, even without instruction, instinctively self-monitor fetal movements (Frøen et al., 2008a). When women have concerns about fetal movements, the complaint may be expressed in vague terms, it may relate to the quality of the movements, a change in pattern (Goodlin, 1979), or constitute a lack of movements at an expected time of day or situation (Malm et al., 2010). In this study, patterns of fetal activity reported by women followed a circadian rhythm characterised by infrequent movement during the morning, increasing likelihood of activity in the afternoon, and high levels of movement in the evening. Bouts of activity or rest were further influenced by the woman's position or by ambulation and rest. Possible explanations for these patterns and their potential clinical significance will be discussed in Chapter 5. 


\section{Chapter 5}

\section{Discussion}

\subsection{Overview of discussion}

This discussion chapter pulls together the various threads of the thesis into a conclusion, firstly by revisiting the impetus for the study: the clinical problem of maternal perception of reduced fetal movements and fetal compromise. A discussion of the findings in relation to the literature will follow, including an appraisal of the study methods, strengths and weaknesses. Finally, thoughts for future avenues of investigation and concluding thoughts are offered.

\subsection{Fetal movements and stillbirth}

Fetal activity is considered a sign of fetal life, and of normally developing fetal neurological, cardiovascular and musculoskeletal systems. In response to hypoxia or malnutrition the fetus reduces movement in order to reduce demand for oxygen and energy (Warrander et al., 2012). In more than half the cases of intrauterine fetal death, the mother reports reduced fetal activity prior to diagnosis (Efkarpidis et al., 2004). In addition to stillbirth, women who present with decreased fetal movements also have a higher incidence of fetal growth restriction and preterm birth (O'Sullivan et al., 2009; Tveit et al., 2009). However, management of these cases is problematic. Complaints about reduced fetal movement are not uncommon, occurring in between $4-15 \%$ of pregnancies (Heazell et al., 2008). Stringent assessment of cases can lead to increased rates of intervention risking iatrogenic harm (Skornick-Rapaport et al., 2011). Conversely, late or limited assessment can mean women go on to experience avoidable stillbirth (Frøen, 2004).

Stillbirth rates remain high, even in developed countries (Flenady, Middleton, et al., 2011). Unexplained intrauterine fetal death occurs too often in 
pregnancies otherwise deemed to be low risk. Improved methods for identifying the fetus at risk are called for (Flenady, Middleton, et al., 2011; Smith, 2011).

\subsection{Maternal perception of fetal movements}

The possibility of fetal movement monitoring to reduce fetal death was first raised by Sadovsky and Yaffe following an observation that pregnant women often reported reduced movements prior to fetal death (Sadovsky \& Yaffe, 1973). Yet research into methods of fetal movement screening has been limited primarily to quantification of fetal movements via kick counting. Descriptive accounts of fetal movements by pregnant women have rarely been sought. Formal fetal movement monitoring has not been proven effective in high quality studies (Mangesi et al., 2007). Methods of monitoring fetal activity are limited by the broad range of fetal movement levels recorded by pregnant women and failure to establish a lower limit of fetal activity. Appropriate methods for evaluation cases of reduced fetal movements are also yet to be established (Hofmeyr \& Novikova, 2012).

Recently a shift has occurred from emphasis on numerical limits of maternally-perceived fetal activity to considering the mother's subjective impression of a change or reduction (Frøen et al., 2008b; Preston et al., 2010; Tveit et al., 2009). Although maternal qualitative perception of a reduction in fetal movements is considered to supersede in clinical importance any quantitative definition (Preston et al., 2010), the nature of qualitative perception of fetal movements by the pregnant woman is under-investigated (Rådestad \& Lindgren, 2012; Raynes-Greenow et al., 2013). This study seeks to provide a qualitative account of fetal movements over the third trimester of pregnancy, according to pregnant women themselves.

\subsection{Review of findings}

The following section comprises a discussion of the key findings of the study and possible implications for further research. The findings have been presented in three sections; the first being a chronological account of fetal movements as perceived by the woman as pregnancy progresses; the second 
explores a novel finding of fetal-activity changes in association with maternal hunger and eating; and the third considers patterns of fetal activity as described by participants. In this chapter the findings are discussed in relation to the literature.

\subsubsection{Fetal movements with advancing gestation - discussion}

In this study, a broad range of properties were accounted for in participant descriptions of fetal movements including: numerous fetal actions, variation in strength, responses to maternal activity and position changes, diurnal variation and responses to external stimuli. These variations came to be an expected part of fetal activity for the pregnant women and were spontaneously monitored by participants in self-devised qualitative fetal movement assessment.

\subsubsection{Fetal movement quality}

This study demonstrated that fetal activity as perceived by the pregnant woman changes over the course of pregnancy. According to participants, fetal movements increased in frequency, strength and variation until 28-32 weeks. Thereafter, kicking and jerking movements reduced, and pushing and stretching movements dominated. At term the pattern was less chaotic, more controlled. Frequency of movements was not reduced in the majority of cases $(61.1 \% n=11)$, but strength was likely to be increased. Maternal perception of a reduction in frequency of fetal movements at term was not uncommon (38.9\%), but was in every case associated with either customised birth weight of the infant below the $25^{\text {th }}$ centile, maternal obesity or operative delivery for fetal indications.

Changes in fetal movement types with advancing gestation were homogenously described by women in this study and are consistent with both ultrasound studies and other qualitative studies of fetal movement perception. A number of features of fetal activity not relating to frequency were spontaneously monitored by women in this study and thought to be reassuring, including increasing strength of movements, bouts of increased activity in the evenings or following a period of ambulation, and movements in response to environmental stimuli such as noise and touch. Response to sound as a stimulus has been proposed as a test of fetal wellbeing, whilst response to touch has been 
documented since the earliest ultrasound studies of fetal movements (Reinhold, 1973).

Quality of fetal movements is increasingly emphasised by neurologists as important in determining normal fetal neurological status (Kurjak et al., 2013; Prechtl \& Einspieler, 1997; Visser et al., 2010). Aspects of fetal activity thought to be indicative of pathology include lower amplitude of movements, sluggish or stereotyped movements (Martin, 2008), persistence of jerky or spastic movements at late gestations, abnormalities in sleep/wake cycling (Martin, 2008) and lack of smooth complex general body movements (Bekedam et al., 1985; Kurjak et al., 2013; Prechtl \& Einspieler, 1997). Fetal movements persist for some time in the hypoxic fetus (Martin, 2008), but might in theory be perceived by the mother to be qualitatively different. Maternal monitoring of normal fetal activity may account for a broad range of fetal functions, possibly explaining the increased specificity of maternal perception of reduced fetal movements in predicting stillbirth.

\subsubsection{Fetal movement types}

In this study, types and patterns of fetal movements were described by pregnant women in a relatively homogenous way. Maternal descriptions of fetal activity with advancing gestation are consistent with fetal movements as documented in ultrasound studies. Ultrasound studies demonstrate that over the first half of pregnancy fetal movements increase in frequency and in variation as increasingly complex movements are added to the repertoire (de Vries et al., 1982, 1985; Marsal, 1983). Jerky or spastic movements are described in ultrasound studies as a key feature of early-term pregnancy, subsiding at term. The overall character of fetal activity with advancing gestation is defined by a shift from chaotic to organised movements (Birnholz et al., 1978; D'Elia et al., 2001; Groome et al., 1998). In keeping with this increasing organisation of fetal activity, longer quiescent periods become apparent and movements are increasingly confined to bursts between rest periods (D’Elia et al., 2001; Pillai et al., 1992). In this study women commonly reported long periods of inactivity at term, 
interspersed by groups of strong movements. Episodic cycling between periods of activity and rest are established by 36 weeks and thought to be an indicator of normal neurological development, persisting into the newborn period (Pillai \& James, 1990; Robertson \& Dierker, 2003). Ultrasound studies have also demonstrated that the fetus has a diurnal pattern to its movements, with increased frequency of movement in the evening and a greater tendency to quiescence during the day (Marsal, 1983; Patrick et al., 1982), which is consistent with the active times of day described by participants in this study.

At term, women in this study described fewer 'kicks', but due to the increase of other movement types (such as pushing, nudging and stretching) the majority found no reduction in movements overall. This is a change which is consistent with other studies (Rådestad \& Lindgren, 2012; Raynes-Greenow et al., 2013). The use by health professionals of the word 'kick' as a catch-all term for fetal movements should therefore be discouraged, as for women the term 'kick' largely refers to just one type of movement, ie a brisk extension and retraction of a foot, and may lead to a mis-reporting of reduced frequency of movements at term (Rådestad \& Lindgren, 2012). Pushing of the fetal legs, in a bilateral leg extension, as described by participants in this study, has been observed on 4D ultrasound to be a frequently undertaken movement by the term fetus. These pushing movements are thought to be similar to those undertaken by the chick in preparation for hatching and may have a similar function in facilitating birth in humans (Andonotopo et al., 2004) .

Maternal descriptions of types of fetal movements and their development over the course of the pregnancy outlined in this study bear a remarkable congruence with that described in other studies of maternally perceived fetal movements suggesting that midwives and other professionals involved in perinatal care may confidently discuss normal fetal activity in qualitative terms with women and expect to be understood.

\subsubsection{Strength and frequency of fetal movements at term}


The question of the importance of reduced fetal movements at term remains. In this study a relative reduction in frequency of fetal movements at term was reported by $38 \%$ of women in this study, which was associated with lower mean customised birthweight, maternal obesity and/or surgical delivery for fetal indications. It must be noted that reduced frequency of movements in this sample was determined from qualitative analysis of the interview transcript data and should not to be confused with a maternal report of concern about reduced fetal movements, which is likely to be more pronounced.

A reduction in frequency of fetal movements at term by obese participants in this study is in keeping with the increased incidence of maternal obesity amongst women presenting with a complaint of decreased fetal movements (Tveit et al., 2009). The reason for a perception of decreased fetal movements amongst obese pregnant women has not been established, however it has been hypothesised that increased adipose tissue might interfere with maternal perception of movements (Tuffnell et al., 1991). In this study obese women reporting a decrease in fetal movements at term, were comparing the movements to those they felt some 10-12 weeks earlier. They were in a sense, acting as their own control and therefore the relative reduction in frequency of movements would seem unlikely to be due to the mechanics of their adiposity, given that none of these women had excessive weight gain over that period. Another possibility may be that fetuses of obese women in this study were less active at term due to the higher maternal blood glucose levels that these women were likely to have had. Lower levels of fetal movement have been reported in diabetic women (Allen \& Kisilevsky, 1999; Devoe, Youssef, Castillo, \& Croom, 1994). Whilst fetal inactivity has been shown to be predictive of fetal macrosomia (Zisser, 2006). Maternal obesity is associated with increased blood glucose levels, even in the non-diabetic (Higgins, Greenwood, Wareing, Sibley, \& Mills, 2011; Whitaker \& Dietz, 1998). Maternal blood glucose levels also increase as pregnancy advances being higher at term (Parretti et al., 2001). Given the prevalence of maternal obesity and the known fetal effects including increased likelihood of aberrant fetal growth (small for gestational age, or fetal macrosomia) and stillbirth, it is important that the phenomenon of reduced fetal activity perception amongst obese pregnant women be further explored. 
Increased strength of fetal movements at term was commonly described by participants in this study, with just $16.7 \%$ of women reporting strength to be mildly reduced at term. In a cross-sectional survey of maternal perception of fetal movements $70 \%$ of respondents reported an increase in strength of movements in the preceding two weeks, regardless of gestation (Raynes-Greenow et al., 2013). In a case control study of late stillbirths increased strength of movements was reported by $36.0 \%$ of control women at term compared to only $14.6 \%$ of women who experienced a stillbirth at term (Stacey et al., 2011a). Increased strength of fetal movements may well be considered a normal and expected feature of maternal perception of fetal movements at term. In the present study no woman who reported increased strength of fetal movements at term delivered a baby under the $20^{\text {th }}$ centile. Increased strength of movements at term may therefore be an indicator of fetal size and vigour, which in turn are indicative of fetal wellbeing.

\subsubsection{Fetal response to maternal hunger and satiation}

Increased fetal activity in association with meals was commonly reported by participants in this study. Anecdotal reports of such activity are not new, and a number of studies explored this possibility in the 1970s through until the early 1990s. The majority of such studies hypothesised that fetal activity was increased following a glucose load and results of investigations to date have been confounding. Some investigators have concluded that increased fetal activity is seen with maternal glucose ingestion (Eller et al., 1992; Miller et al., 1978), whilst others declared no such association (Bocking et al., 1984; Druzin \& Foodim, 1986; Patrick et al., 1982). At this time reviewers consider that there is either no association between fetal activity and maternal meals (Fisher, 1999b), or that the issue is confounding and remains unresolved (Kisilevsky \& Low, 1998; Reece, Coustan, \& Gabbe, 2004). It is possible that the nature of the fetal response to maternal meals has remained elusive to date, due to its complexity and its propensity for variation between pregnancies.

Endocrine studies appear to support, in principle, the phases of fetal activity in response to hunger and satiation as described by participants in this study. An inverse relationship between maternal glucose levels and fetal activity has been established, in that increased fetal activity is seen with maternal hypoglycaemia 
(Holden, 1984; Robertson \& Dierker, 2003), whilst decreased fetal activity is seen with maternal hyperglycaemia (Edelberg et al., 1987).

Fetal activity levels were in relation to maternal blood sugar in 10 diabetic women in the $36^{\text {th }}$ to $40^{\text {th }}$ week of pregnancy, as observed by Holden and colleagues (1984). Contrary to their hypothesis, a statistically significant increase in fetal activity was seen during episodes of hypoglycaemia (Holden, 1984). Another group, using the glucose clamp technique, demonstrated that a significant decrease in fetal movements was associated with sustained maternal hyperglycaemia in a group of nine healthy pregnant women when compared to six controls (Edelberg et al., 1987).

A 1992 study in the U.S.A. attempted to show an increase in fetal activity following a glucose load by studying fetal activity levels amongst 21 pregnant women undergoing glucose tolerance testing (GTT), compared to 21 fasted controls at the same gestation. Baseline fetal activity levels were established in the women 10 minutes prior to their fasting blood glucose measurement. An increase in fetal activity levels was seen in the glucose group, but progressively increasing levels of fetal activity over time were also seen in the control group (Eller et al., 1992). The on-going fasted state of the control participants was overlooked as a possible explanation for increasing levels of fetal activity in this group, leaving the investigators at a loss to explain how fetal activity had increased in both groups.

A study of cyclic motor activity in fetuses of pregnant women with Type 1 or gestational diabetes in relation to blood-sugar level demonstrated that fetal activity became faster when maternal blood-sugar levels decreased, and slower when maternal blood-sugar levels increased. Interestingly, the authors did not observe any relationship between fetal cyclic motility and maternal blood glucose levels per se. The key relationship noted was the change in fetal activity levels in response to change in maternal blood-sugar levels (increasing or decreasing) rather than the level itself (Robertson \& Dierker, 2003).

Patrick and colleagues (1982) observed the gross fetal body movements of 31 pregnant women between 30 and 39 weeks gestation by continuous ultrasound recording over a 24-hour period. Fetal activity levels were seen to increase over 
the late evening, peaking at midnight, the same time period in which maternal blood glucose levels progressively dropped as the women went into their nighttime fast (Patrick et al., 1982).

The effect of maternal glucose on fetal breathing movements is well established. Controlled studies have demonstrated that administration of a glucose load to pregnant women results in an almost two-fold increase in fetal breathing movements (Bocking et al., 1984; Goodman, 1980). Increases in fetal breathing movements are more often observed during a resting state in the fetus, whilst gross fetal body movements occur more often in periods of apnoea (Marsal, 1983). Increased fetal breathing movements in association with higher maternal bloodsugar levels, provides support in principle for the notion of fetal quiescence post-prandially reported in this study.

Patterns of ingestive behaviour have been established in animals and humans (Strubbe \& Dijk, 2002; Watts, 2000) and are homologous to those recounted by participants in this study in relation to fetal activity around meals. In animal studies the period prior to meals is characterised by a gradual crescendo of increasing activity. Foraging animals may spend a considerable period of time procuring food in this phase. Following consumption of a meal, animals will generally become distracted from eating once they are sated and may spend a short period grooming or engaged in other activities before settling down for a sleep (Antin et al., 1975; Berthoud, 2002). The time period for this transition varies between individuals but is generally one of increased activity in the procuring of food phase and rest in the post-prandial phase.

Glucose is the principle source of energy for the fetus, and as such glucose and insulin are likely to be the key mediators in any fetal response to hunger and satiation. Yet, moderation of hunger and satiety in humans and animals is extremely complex, involving many hormones and metabolites in addition to glucose and insulin. It is interesting to note that behaviour suggestive of hunger and satiation in the fetus would not be subject to any gustatory inputs given that the fetus naturally does not have gut feeds, emphasising the primacy of neuroendocrine inputs in the development of hunger and satiety. 


\subsubsection{Mood and food}

Maternal descriptions of fetal activity at mealtimes in this study emphasised that fetal activity was indicative of fetal mood in relation to prandial state, especially 'frustration' at being hungry and 'excitement' or 'contentment' at being fed. The connection between feeding and the brain has been acknowledged since the Russian physiologist Pavlov demonstrated anticipation of food by salivation in dogs exposed to the stimulus of a ringing bell (Pavlov, 1927). Hormones involved in hunger and satiety have known central-nervous-system effects, including acting on parts of the brain controlling learning and emotion (Bertoud, 2008). The empathy displayed in the participant's appreciation of her baby's emotional state in relation to hunger and satiation might be considered an example of pre-birth formation of a mother-infant bond. Maternal-infant interaction and especially maternal responsiveness to infant cues, are essential to growth and development of infants after birth (White, Simon, \& Bryan, 2002). DiPietro (2010) asserts that the development of a mother-infant bond begins well before birth and is mediated by the hormonal milieu, with active inputs into the shaping of the intra-uterine environment coming from both the mother and the fetus, 'While the uterus is the developmental niche of the fetus, it has become increasingly clear that the fetus is also an active inhabitant of that niche.' (DiPietro, 2010, p.35). It is not unreasonable to consider increased fetal movements in the context of hunger as fetal communicative input, a fetal demand for food.

\subsubsection{Fetal food-seeking: an evolutionary adaptation?}

For much of our existence as a species, humans have survived in environments of food scarcity. It is plausible that a fetal response to low energy supply may have evolved as a mechanism for ensuring optimum availability of nutrients to maintain pregnancy. John Bowlby (1969) in his study of mother-infant bonding coined the term 'Environment of Evolutionary Adaptedness' (EEA), tracing many infant responses to the conditions for survival in our nomadic huntergatherer past (Bowlby, 1969). In Bowlby's theories of attachment, maternal and infant interests are often dovetailed (Ainsworth \& Bowlby, 1991). Maternal 
receptiveness to a fetal mechanism for prompting eating where energy supplies are low, could arguably serve not just the interests of the growing fetus but also the pregnant woman, who optimally nourished will better withstand labour, birth and the subsequent demands of lactation.

\subsubsection{Fetal activity, fetal growth and stillbirth}

In this study, maternal reports of increased fetal activity in the context of hunger were associated with smaller size of the baby at birth. Both small and large fetal size is associated with increased risk of stillbirth (Huang et al., 2000) and any relationship between fetal activity and birthweight warrants further exploration. Animal studies demonstrate that in the context of placental insufficiency, fetal metabolic changes such as increased insulin precede a drop-off in fetal growth (Limesand et al., 2012). In intra-uterine growth-restricted (IUGR) pregnancies there is a larger maternal-fetal glucose concentration gradient, with the IUGR fetus being relatively hypoglycaemic (Hay, 2006). It may be that fetal responses to maternal hunger and satiation were preferentially reported in this study by women who later delivered small babies, as these fetuses were more sensitive to changes in maternal glucose due to metabolic adaptations to an environment of reduced energy supply via the placenta.

The relationship between IUGR, stillbirth and decreased fetal movements is well documented (Warrander \& Heazell, 2011; Winje, Roald, Kristensen, \& Frøen, 2012). It has been hypothesised that decreased fetal movements might be a compensatory measure to reduce energy expenditure in the context of placental insufficiency (Warrander \& Heazell, 2011). In this study increased fetal activity in the context of maternal hunger was interpreted as a demand for food. The small infants in this study were well newborns with normal Apgar scores, who followed a normal neonatal course. Hypothetically, fetal food-seeking behaviour might be a compensatory mechanism employed by a healthy fetus in response to low fuel supply. Decreased fetal movements in the context of fetal growth restriction or severe malnutrition might thus represent a de-compensation or loss of foodseeking response where energy supply has become severely diminished. In a 
study of 26 women who had experienced stillbirth, 22 reported a premonition that something had happened to their baby prior to diagnosis of fetal death. A key category emerging from interviews with those mothers was 'losing contact with the baby'. Losing contact was frequently exemplified by mothers as absence of expected movements at mealtimes (Malm et al., 2010).

\subsubsection{Fetal movement patterns in relation to meals}

Temporal fluctuations in fetal activity in this study were described by many participants as being closely related to meals. Temporal aspects of fetal activity are of interest as a possible source of clues as to how fetal activity changes might warn of impending stillbirth (Winje, Røislien, et al., 2012). It has been established that human fetal activity has a complex temporal nature in that alternating bouts of activity and rest occur throughout the day and night, with increased activity over the evening period and increased incidence of quiescent periods during the day (Pillai et al., 1992). Yet the mechanisms for controlling these fluctuations are not understood. Consideration of the potential influence of energy supply via the placenta as a driver of temporal fluctuations in fetal activity is warranted. The diurnal fetal activity pattern is periodically abolished following administration of steroids to pregnant women for fetal lung maturation (de Vries, Visser, \& Prechtl, 1988). This response may well be due to the disruption to maternal glucose regulation that occurs with this therapy.

Patterns of fetal activity are known to be altered in pregnancy pathologies which also involve alterations in energy supply to the fetus, including IUGR and diabetes. Abnormalities in behavioural state cycling precede abnormalities in general movement quality in growth-restricted fetuses (Sival et al., 1992a), whilst fetuses of diabetic women exhibit delayed behavioural state organisation (Mulder, Visser, Bekedam, \& Prechtl, 1987) and changes in fetal behaviour are seen on ultrasound prior to evidence of 'brain-sparing' (Kurjak et al., 2013). It has been hypothesised that these changes are indicative of fetal neuro-developmental delay; however, they might just as likely be indicative of altered metabolic environment in these pregnancies (Allen \& Kisilevsky, 1999). 


\subsubsection{Fetal quiescence postprandially}

Reporting of fetal quiescence in the context of the maternal postprandial state by some participants in this study raises the possibility that increased glucose might have a suppressive effect on fetal activity. Glucose metabolism requires consumption of oxygen. A study of fetal glucose metabolism in lambs demonstrated that fetal oxygen consumption is almost entirely accounted for in the metabolism of glucose supplied by the mother (Hay, 2006). Oxygen requirements for glucose metabolism, either in the postprandial period or where maternal glucose is chronically elevated, might therefore compete with oxygen requirements for movement in the fetus.

Decreased fetal movements are more frequently reported by overweight or obese pregnant women (Heazell \& Frøen, 2008; Tveit et al., 2009). Although the cause for this is unknown, it has been postulated that obese women may have decreased sensitivity to fetal movements due to excess adipose tissue, reducing the impact of any fetal movement impulse on maternal skin (Tuffnell et al., 1991). The possibility of actual reduction in fetal movements in obese women during pregnancy, as opposed to reduced perception has not been ruled out. Studies have shown fetal activity is reduced in pregnancies complicated by diabetes (Allen \& Kisilevsky, 1999; Devoe, Youssef, Castillo, \& Croom, 1994). In diabetic pregnancies resulting in fetal hyperglycaemia and hyperinsulinaemia, the risk of fetal hypoxemia and acidaemia are increased due to competing oxygen requirements (Reece et al., 2004). Maternal obesity is known to be associated with increased blood-glucose levels in pregnancy (Higgins et al., 2011; Whitaker \& Dietz, 1998). Where the obese woman's metabolic disturbances do not meet levels diagnostic of gestational diabetes, the fetus may nonetheless be exposed to an augmented glucose supply and an increased oxygen demand.

In a study of 46 diabetic pregnant women, a relationship between fetal activity levels and subsequent birthweight was established (Zisser, 2006). Glucose-mediated macrosomia was seen exclusively amongst fetuses prospectively determined to be 'inactive'. The authors postulated that the reason for the observed relationship between fetal activity and birthweight in babies of their participants may be an intrinsic tendency for the smaller babies in their study 
to be more active (the fidgety fetus hypothesis), which protected them from macrosomia (Zisser, 2006). However, they also demonstrated that higher birthweight of the infants in their study was correlated with higher maternal postprandial blood-glucose levels $(r=0.704)$. An alternative or supplementary explanation for their finding is that fetal activity levels provided a sensitive indicator of maternal glucose levels; a relationship borne out in the infant's later birthweights.

Familiarity with prior published research concluding that fetal movements are not influenced by meals effectively blinded the researcher to this finding until all but the final stages of data gathering. The majority of participants were not asked directly if their baby moved at mealtimes, but instead volunteered the descriptions when asked if there were any times their baby moved more or less than usual. The finding has thus emerged unsolicited.

\subsubsection{Temporal, positional and kinaesthetic elements}

Patterns of fetal movement are a relatively unexplored aspect of fetal movements that might be of value in understanding changes in the context of compromise (Saastad et al., 2011). In this study, patterns of fetal movement were described by participants with great consistency. The key influences on perception of fetal activity, according to participants were: time of day, position and maternal movement.

Evenings were reported by all participants as a time of increased fetal activity, with smaller peaks reported by many in the early morning and around lunch time. This pattern is consistent with the diurnal fetal movement pattern established in ultrasound studies, lending reliability to the maternal account of fetal activity patterns (de Vries, Visser, Mulder, \& Prechtl, 1987; Patrick et al., 1982). Drivers of the fetal circadian rhythm are unknown but are thought to be entrained by the mother, perhaps working in opposite phase or in a pattern of phase delay following the mother's pattern (Kintraia, Zarnadze, Kintraia, \& Kashakashvili, 2005). Thus the times the fetus is most active are the times the mother is least active. Pregnant women are known to perceive fewer fetal movements when they are active (Frøen et al., 2008b). This is normally explained as a function of the woman's distraction with other tasks or else diminished perception as a result of 
upright posture; however the reports are consistent with the fetal circadian rhythm and therefore may have a physiological basis.

The clinical significance of the fetal circadian rhythm is unknown, but warrants further consideration. One study demonstrated a significant reduction in stillbirths with fetal movement counting, when mothers were specifically instructed to count in the evenings (Moore \& Piacquadio, 1989). This counting method effectively measured the evening burst of fetal movements, which reflects normal fetal circadian rhythm.

Participants in the present study also reported increased fetal activity when they sat or lay down as opposed to when they were upright and moving. This may be due to effects of position on perception of movement, effects of activity and rest on fetal physiology, or may reflect the mother's impression in relation to the fetal circadian rhythm.

\subsubsection{Temporal aspects}

Ultrasound studies of fetal movements have established that fetal activity has a rich temporal structure with alternating periods of activity and rest over minutes and over the 24-hour day (Robertson \& Dierker, 2003). Patterns of fetal activity as described by women in this study in relation to time of day are consistent with the fetal circadian rhythm established in objective methods of fetal activity observation. Ultrasound studies confirm a fetal circadian rhythm, characterised by active periods occurring in the evening and increased incidence of quiescent periods during the day (Carmichael, Campbell, \& Patrick, 1984; Marsal, 1983; Patrick et al., 1982). Maternal reporting of increased evening fetal activity and morning quiescence is not new to this study and was also reported in an Australian cross-sectional survey of fetal movements (Raynes-Greenow et al., 2013) and a British audit of times of day women were more likely to perceive fetal activity (Fisher, 1999a). Maternal reports of fetal movement patterns in relation to time of day should be considered relatively reliable given they are similarly reported across several studies and are consistent with the observed diurnal fetal movement pattern established by objective methods of observation. 
The diurnal pattern of fetal activity is considered to be a normal and reassuring pattern, yet very little is known about how it is driven. Fluctuations in maternal cortisol have been suggested as a driver of the diurnal pattern (Koenen, Mulder, Wijnberger, \& Visser, 2005; Pillai et al., 1992), whilst short term changes in fetal activity might reflect adaptive responses to the environment (Robertson \& Dierker, 2003). Circadian rhythms are a pervasive feature of mammalian physiology and have been observed in a number of fetal animal species in addition to humans (Stark, Garland, Daniel, Tropper, \& Myers, 1999). Circadian rhythms are primarily endogenous but are also influenced by environmental factors such as light and dark. Although the fetus has a separate brain, endocrine and circulatory system from that of its mother, it is tethered to the environment provided by the mother in a profound way. It is therefore not unlikely that the metronome of fetal fluctuations in periods of activity and rest is set by the mother. In the present study, women commonly described their own inactivity as promoting fetal movement and vice versa. The fetal circadian rhythm is thought to be entrained by the mother (Stark et al., 1999; Van Leeuwen, Geue, Lange, \& Gronemeyer, 2009; Van Leeuwen et al., 2003). Daily rhythmicity of fetal heart rate and locomotor activity was established in a study of more than 2500 fetuses where periods of activity and rest were demonstrated to have a diurnal rhythm that resembled that of the mother's, except that it ran in an opposite phase so that active periods for the mother were quiet periods for the fetus and vice versa (Kintraia et al., 2005). It is plausible that maternal activity, rest, sleeping and eating may all provide inputs influencing fetal patterns of activity and rest.

Appreciation of the fetal-activity circadian rhythm may be useful for midwives and obstetricians, particularly given that it is consistently reported by mothers. Encouraging women to report for assessment if their baby has not moved sufficiently by a set hour of the afternoon, means the evening period of increased activity is not accounted for and there is greater likelihood of quiescent periods whilst the woman is upright and active. This approach has not been proven effective and risks unnecessary worry for the woman and inappropriate use of resources (Fisher, 1999b). For the woman who is not normally aware of fetal activity over the course of the morning but experiences vigorous activity in the evening, daytime quiescence is not likely to be a problem. Conversely, evening or night-time reports of reduced fetal movements may be inconvenient for clinicians, 
requiring out of hours assessment, but they are likely to be unusual for the woman and warrant investigation. Of course each pregnancy is unique and the times of day that the fetus is perceived to be active will vary between pregnancies. It is important when discussing fetal activity with pregnant women to consider both the normal pattern and what is usual for her.

\subsubsection{Maternal positional aspects}

In this study a fetal-maternal interaction was evident in participants' descriptions of fetal responses to maternal position changes. Fetal activity was reported to increase in response to the mother adopting a sitting or lying position or following the mother rolling over in bed. In other instances the reverse occurred and the mother was prompted to change her position in response to a perceived demand on the part of the fetus. Some maternal positions may be better for the fetus than others. Maternal position changes may impact on the fetal state by impairing oxygenation such as compression of the inferior vena cava when supine or by reducing available space for spontaneous movement. In a case control study of modifiable risk factors for stillbirth, maternal back sleeping position was strongly associated with increased risk of stillbirth (Stacey et al., 2011). Spontaneous fetal movement is necessary for development of the fetal limbs. A vigorous fetal response was often reported by women in this study who adopted a position that was cramped or otherwise reduced space in the abdomen. A maternal-fetal interaction via fetal movements may be an important feature of normal pregnancy, indicating a fetal contribution to maintenance of homeostasis in the intra-uterine environment.

In the present study participants described increased intensity of fetal movements when lying down as compared to sitting, and reduced intensity when upright as compared to sitting. The association between maternal position and fetal movements was first described in a study published in 1976 where six pregnant women kept diaries of fetal activity, noting times of day fetal movements were perceived and the woman's position at the time (Minors \& Waterhouse, 1979). Analysis of the data concluded that lying was associated with increased fetal movement, sitting less so, and standing with the least movements. A 
weakness in Minors and Waterhouse's study is that maternal ambulation was not accounted for. Participants in that study had just three options for recording their position in relation to fetal movements: sitting, standing and lying, all of which are static. As the subjects were required to document both their own activity and the activity of the fetus as they went about their day, it is more than likely that when the women were upright they were also moving. A subsequent study involving 160 women recorded numbers of fetal movements perceived during sequences of posture changes. Participants went through six possible sequences of sitting, standing or lying. The sequence that produced the largest number of movements was standing-sitting-lying $(P<0.04)$. Significantly fewer movements were recorded in the standing position relative to the other two $(P<0.01)$. The authors hypothesised that reduced perception of movement in the standing position might be due to relatively lower perfusion of the uterus in the upright position, whilst the high numbers of movements recorded in the standing-sitting-lying sequence might be due to 'compensatory hyperactivity of the fetus after moving from the initial unfavourable posture' (Zimmer et al., 1982, p. 86). Bouts of increased activity when sitting following a period of ambulation were universally reported by women in this study.

\subsubsection{Kinaesthetic elements}

It is widely acknowledged that pregnant women may report a perception of decreased fetal movement when they are busy (Moore \& Piacquadio, 1989; Unterscheider \& O'Donoghue, 2012; Whitworth et al., 2011). These reports have not been the subject of any significant research attention to date and are presumed to be a misperception based on upright position (Whitworth et al., 2011) or a function of maternal attention to things (Raynes-Greenow et al., 2013). In the present study, maternal activity was explicitly described by pregnant women as having a suppressive effect on fetal movements, effectively 'rocking the baby to sleep', whilst maternal rest was described as 'waking the baby' and precipitating a period of increased fetal activity. Fetal responses to numerous aspects of maternal physiology have been documented, including maternal exercise (Jovanovic, Kessler, \& Peterson, 1985; Manders, Sonder, Mulder, \& Visser, 1997), glucose 
(Edelberg et al., 1987; Robertson \& Dierker, 2003) and maternal breathing rates (Van Leeuwen, Geue, Thiel, et al., 2009). It is not implausible that the fetus is sensitive even to small changes in maternal physiology in association with walking around or resting.

There is an impressive continuity between newborn behaviour and that observed in the term fetus, in that barely any behaviours exhibited in the newborn cannot also be identified in the fetus (Eispeiler et al., 2008). Any new parent will attest to the newborn need to be jiggled and paced about with when unsettled. Receptiveness to the soothing effect of being in the arms of a pacing caregiver may originate before birth where the ambulant mother's movements had a similar effect on the fetus.

Determining whether fetal movement levels are influenced by maternal activity and rest or simply perceived to be so, is problematic. Objective methods of fetal movement recording generally require the mother to be still and preferably recumbent, making evaluation of fetal activity levels in the ambulant mother technically difficult. The perception by participants in this study that their activity fostered fetal rest and vice versa may have been an impression gained in relation to the fetal circadian rhythm, or indeed relate to sensitivity to movements due to maternal position. However, the possibility that short-term fetal transitions between active and quiet periods may be influenced by changes in the mother's activity and rest warrants further investigation, given that it appears to be a significant feature of normal fetal activity as described by women. Women in this study reported being reassured when their baby became active after they sat or lay down. The maternal account of fetal activity appears to be a dynamic one that accounts not only for fetal life but fetal neurological and central nervous system function in observing appropriate fetal responses to environmental factors.

\subsection{Appraisal of study methods}

Qualitative description is a qualitative methodology that emphasises description over theory or interpretation. The methodology is useful in health applications where a first-hand patient account is sought. An emphasis on description rather than interpretation means there is no need to push for a 
univocal account and variation can be embraced, making it an appropriate methodology for investigation of maternal perception of fetal movements. The impetus for this study was to explore maternal perception of fetal movements in the hope that these descriptions might provide clues for later studies of fetal movements in relation to stillbirth prevention. The study employed a complementary design where the qualitative analysis was followed by quantitative evaluation of fetal movement patterns reported by participants and subsequent outcomes. Health-care researchers have a particular interest in such mixed approaches, perhaps in acknowledgement of the complex nature of health problems (Morgan, 1998).

\subsubsection{Study strengths}

Strengths of this study include the novel design, involving prospective gathering of qualitative descriptions of fetal movements, with variations considered according to later outcomes.

Strengths of the study include investigation of a qualitative phenomenon using qualitative means, and the novel approach of considering prospectively gained qualitative accounts alongside later outcomes. The inductive approach allows for the emergence of serendipitous findings such as the complex pattern of fetal activity in relation to maternal meals. A small number of papers investigating qualitative perception of fetal movements by pregnant women have been published since the inception of this study (Rådestad \& Lindgren, 2012; RaynesGreenow et al., 2013). Participant descriptions in the present study are consistent with descriptions reported in other studies, lending validity to the findings.

\subsubsection{Study weaknesses}

A limitation in the study is the selection criteria. Participants were to be lowrisk women in their first pregnancy, so that a baseline understanding or normal fetal activity could be gained. The criterion for participant eligibility was absence of conditions warranting referral to a specialist at the time of booking. This group was thought to be representative of a normal case-load for a primary care Lead Maternity Carer in New Zealand. However, fetal death often occurs in women not 
previously identified as high risk. There are a number of risk factors associated with stillbirth that can exist in women designated as suitable for primary care, such as maternal overweight and maternal smoking (Stacey et al., 2011b). For this study a more stringent definition of low risk might have yielded different results. For example, approximately one third of participants reported fetal movements to be less frequent at term, yet these women were in all cases either overweight, delivered a baby under the $25^{\text {th }}$ percentile or required expedited delivery for fetal distress which in some studies would not have been considered low-risk.

Participants in this study were primiparous women who had agreed to be part of a study of fetal movements, and for these reasons it is possible participants were more attentive to fetal movements. Further, the sample size was small. Whilst this made in-depth analysis of qualitative data feasible, statistical associations reported should be interpreted with caution.

\subsection{Research Implications}

Maternal descriptions of fetal activity with advancing gestation were found to be relatively homogenous within the participant sample and also consistent with maternal accounts of fetal activity published since the study's inception (Rådestad \& Lindgren, 2012; Raynes-Greenow et al., 2013). Qualitative aspects of maternally perceived fetal movements are valued by health professionals but have not been explicitly evaluated in screening tools. Future research might consider evaluation of qualitative aspects of fetal movements in screening tools. Quality of fetal movements provides an important indicator of fetal neurological status and has been shown to be altered in the context of numerous pregnancy pathologies. The relative consistency of fetal movement descriptions suggests these terms may be used for discussions between pregnant women and midwives.

In this study a perception of fewer movements at term was associated with maternal obesity, birth weight under the $20^{\text {th }}$ centile and fetal indications for delivery (Table 4). Decreased fetal movement is known to be associated with maternal obesity. This association is problematic, as it remains to be determined whether movement perception is reduced in the context of obesity or whether actual fetal activity is reduced. Overweight and obese women have higher rates of fetal growth problems and of stillbirth. Further research should consider the 
possibility of maternal obesity altering fetal movements and any screening implications specifically for this group.

Human hunger and satiation is a subject of great importance in the current environment of pandemic obesity. Behaviour suggestive of hunger and satiation in the fetus has not previously been reported, but may be valuable when it is considered that the environment of the womb is thought to influence later incidence of obesity of offspring (Whitaker \& Dietz, 1998). Investigation of physiological mechanisms governing fetal responses to maternal meals may prove useful in improving understanding of the origins of human variation in hunger, satiety and inclination for activity. The association between smaller size at birth and increased fetal activity prior to meals reported in this study must be treated with caution due to the small numbers of women in the study. Future studies might consider objective evaluation of fetal movements over the course of a meal, prior, during and post, according to pregnancy characteristics. Identification of the hormones involved in fetal responses to maternal hunger and satiation may further understanding of fetal growth problems.

Further investigation of the physiological basis of the fetal circadian rhythm is warranted, given the importance of this aspect of fetal activity in maternal accounts. Normal patterns may represent normal placental function and transport of pattern modulators from the maternal circulation. Fetal movement monitoring tests should be designed to take into account the fetal circadian rhythm.

\subsection{Conclusion}

This study provides a qualitative account of fetal movements as perceived by low-risk primiparous women during the third trimester. In this study, fetal movements were found to act as a form of communication between mother and fetus: increased activity might prompt the mother to eat when hungry, and change position when her posture was overly restrictive, thereby maintaining optimal conditions for the pregnancy. Patterns of fetal activity and rest described by mothers in this study were closely tied to maternal activity and rest in an inverse relationship, suggesting that patterns of fetal activity may be influenced by the environment. Women in this study instinctively interpreted their baby's pattern as normal, and monitored their own baby according to expected movements patterns 
and responses to environmental conditions. Maternal monitoring of fetal movements may account for a broader range of fetal functions than simply the ability to move, explaining their specificity in heralding fetal jeopardy.

It is difficult to imagine a closer association between two people than that of the pregnant woman and her growing fetus. The maternal account of fetal activity may have special importance as the account of the verbal partner engaged in a unique dyadic interaction with the fetus. The fact that women often become aware of changes in fetal movements prior to overt clinical signs of compromise is hardly surprising and may be a powerful tool in understanding life before birth. In the quest to further efforts for stillbirth preventions this study provides no answers. But it does raise some compelling questions. 


\section{References}

Ahn, M. O., Phelan, J. P., Smith, C. V., Jacobs, N., \& Rutherford, S. E. (1987).

Antepartum fetal surveillance in the patient with decreased fetal movement. American Journal of Obstetrics and Gynecology, 157, 860-864.

Ainsworth, M. D., \& Bowlby, J. (1991). An Ethological Approach to Personality Development. American Psychologist, 46(4), 333-341.

Allen, C., \& Kisilevsky, B. (1999). Fetal behavior in diabetic and nondiabetic pregnant women: an exploratory study. Developmental Psychobiology, 35(1), 69-80.

Allen, M. C. (2002). Preterm outcomes research: A critical component of neonatal intensive care. Mental Retardation and Developmental Disabilities Research Reviews, 8(4), 221-233. doi:10.1002/mrdd.10044

Andonotopo, W., Stanojevic, M., Kurjak, A., Azumendi, G., \& Carrera, J. (2004). Assessment of fetal behavior and general movements by four-dimensional sonography. The Ultrasound Review of Obstetrics \& Gynecology, 4(2), 103-114.

Antin, J., Gibbs, J., Holt, J., Young, R., Smith, G., \& Bourne, E. (1975). Cholecystokinin elicits the complete behavioral sequence of satiety in rats. Journal of Comparative and Physiological Psychology, 89(7), 784-790.

Baston, H. (2003). Monitoring fetal wellbeing during routine antenatal care. The Practising Midwife, 6(4), 29-33.

Bazeley, P. (1999). The bricoleur with a computer: Piecing together qualitative and quantitative data. Qualitative Health Research, 9(2), 279-287.

Bekedam, D. J., Visser, G. H. A., de Vries, J. J., \& Prechtl, H. F. . (1985). Motor behaviour in the growth retarded fetus. Early Human Development, 12, 155-165. 
Belizian, J. M., \& Villar, J. (1980). The relationship between calcium intake and edema, proteinuria, and hypertension-getosis: a hypothesis. American Journal of Clinical Nutrition, 33(10), 2202-2210.

Bennet, V. R., \& Brown, L. K. (1999). Myles Textbook for Midwives (13th ed.). Edinburgh: Churchill Livingstone.

Berthoud, H.-R. (2002). Multiple neural systems controlling food intake and body weight. Neuroscience and Biobehavioral Reviews, 26, 393-428.

Bertoud, H. (2008). Vagal and hormonal gut-brain communication: from satiation to satisfaction. Neurogestroenterology and Motility, 20(Suppl. 1), 64-72.

Bhutta, Z. A., Yakoob, M. Y., Lawn, J. E., Rizvi, A., Friberg, I. K., Weissman, E., ... Goldenberg, R. L. (2011). Stillbirths: what difference can we make and at what cost? The Lancet, 377(9776), 1523-1538. doi:10.1016/S0140-6736(10)62269-6

Birnholz, J. C., Stephens, J. C., \& Faria, M. (1978). Fetal Movement Patterns: a possible means of defining neurologic developmental milestones in utero. American Journal of Roentgenology, 130, 573-540.

Bocking, A., Adamson, L., Carmicheal, L., Patrick, J., \& Probert, C. (1984). Effect of intravenous glucose injection on human maternal and fetal heart rate at term. American Journal of Obstetrics and Gynecology, 148, 414-420.

Bondas, T., \& Eriksson, K. (2001). Women's lived experience of pregnancy: a tapestry of joy and suffering. Qualitative Health Research, 11(6), 824-840.

Bowlby, J. (1969). Attachment (2nd ed., Vols. 1-3, Vol. 1). United States if America: Basic Books.

Bucher, H. C., Guyatt, G. H., Cook, R. J., Hatala, R., Cook, D. J., Lang, J. D., \& Hunt, D. (1996). Effect of calcium supplementation on pregnancy-induced hypertension and 
pre-eclampsia: a meta-analysis of randomized controlled trials. JAMA: The Journal of the American Medical Association, 275(14), 1113-1117.

Carmichael, L., Campbell, K., \& Patrick, J. (1984). Fetal breathing, gross fetal body movements, and maternal and fetal heart rates before spontaneous labor at term. American Journal of Obstetrics and Gynecology, 148(5), 675-679.

Cioni, G., Prechtl, H. F. ., Ferrari, F., Paolicelli, P., Einspieler, C., \& Roversi, F. (1997). Which better predicts later outcome in fullterm infants: quality of general movements or neurological examination? Early Human Development, (50), 71-85.

Coppens, M. (2001). Computerized analysis of acute and chronic changes in fetal heart rate variation and fetal activity in association with maternal smoking. American Journal of Obstetrics and Gynecology, 185(2), 421-426. doi:10.1067/mob.2001.115992

Dayal. (1999). Fetal death after normal biophysical profile score: an eighteen year experience. American Journal of Obstetrics and Gynecology.

D’Elia, A., Pighetti, M., Moccia, G., \& Santangelo, N. (2001). Spontaneous motor activity in normal fetuses. Early Human Development, 65, 139-147.

Denzin, N., \& Lincoln, Y. (2011). The discipline and practice of qualitative research (4th ed., pp. 1-19). USA: Sage.

Devoe, L., Youssef, A., Castillo, R., \& Croom, C. (1994). Fetal biophysical activities in third trimester pregnancies complicated by diabetes mellitus. American Journal of Obstetrics and Gynecology, 171(2), 298-303.

De Vries, J. I., Visser, G. H. A., \& Prechtl, H. F. (1982). The emergence of fetal behaviour. I. Qualitative aspects. Early Human Development, 7, 301-322.

De Vries, J. I., Visser, G. H. A., \& Prechtl, H. F. (1985). The emergence of fetal behaviour. II. Quantitative aspects. Early Human Development, 12, 99-120. 
De Vries, J. I., Visser, G. H. A., \& Prechtl, H. F. (1988). The emergence of fetal behaviour. III. Individual differences and consistencies. Early Human Development, 16, 85-103.

De Vries, J. I., Visser, G. H., Mulder, E. J., \& Prechtl, H. F. (1987). Diurnal and other variations in fetal movements and heart rate patterns at 20-22 weeks. Early Human Development, 15, 333-348.

Dierker, L. J., Jr, Pillay, S., Sorokin, Y., \& Rosen, M. G. (1982). The change in fetal activity periods in diabetic and nondiabetic pregnancies. American Journal of Obstetrics and Gynecology, 143(2), 181-185.

DiPietro, J. A. (2010). Psychological and psychophysiological considerations regarding the maternal-fetal relationship. Infant and Child Development, 19(1), 27-38.

DiPietro, J. A., Hilton, S. C., Hawkins, M., Costigan, K. A., \& Pressman, E. K. (2002). Maternal stress and affect influence fetal neurobehavioral development. Developmental Psychology, 38(5), 659-668.

Dipietro, J. A., Irizarry, R. A., Costigan, K. A., \& Gurewitsch, E. D. (2004). The psychophysiology of the maternal-fetal relationship. Psychophysiology, 41(4), 510-520. doi:10.1111/j.1469-8986.2004.00187.x

Druzin, M., \& Foodim, J. (1986). Effect of maternal glucose ingestion compared with maternal water ingestion on the nonstress test. Obstetrics and Gynecology, 67(3), $425-426$.

Dubiel, M., Gudmundsson, S., Thuring-Jonsson, A., Maesel, A., \& Marsal, K. (1997). Doppler velicometry and nonstress test for predicting outcome of pregnancies with decreased fetal movements. American Journal of Perinatology, 14(3), 139-144.

Duden, B. (1992). Quick with Child: an experience that has lost its status. Technology in Society, 14, 335-334. 
Dutton, P. J., Warrander, L. K., Roberts, S. A., Bernatavicius, G., Byrd, L. M., Gaze, D., Heazell, A. E. P. (2012). Predictors of Poor Perinatal Outcome following Maternal Perception of Reduced Fetal Movements - A Prospective Cohort Study. PLoS ONE, 7(7), e39784.

Edelberg, S., Dierker, L. J., Kalhan, S., \& Rosen, M. G. (1987). Decreased fetal movements with sustained maternal hyperglycaemia using the glucose clamp technique. American Journal of Obstetrics and Gynecology, 156, 1101-1105.

Efkarpidis, S., Alexopoulos, E., Kean, L., Liu, D., \& Fay, T. (2004). Case-Control Study of Factors Associated With Intrauterine Fetal Deaths. Medscape General Medicine, 6(2), 53.

Eispeiler, C., Marschik, P., \& Prechtl, H. (2008). Human Motor Behaviour: prenatal origin and early postnatal development. Journal of Psychology, 216(3), 148-154.

Eller, D., Stramm, S., \& Newman, R. (1992). The effect of maternal intravenous glucose administration on fetal activity. American Journal of Obstetrics and Gynecology, 167, 1071-1074.

Ferrari, F., Prechtl, H. F., Cioni, G., Roversi, F., Einspieler, C., Gallo, C., Cavazzuti, G. (1997). Posture, spontaneous movements, and behavioural state organisation in infants affected by brain malformations. Early Human Development, (50), 87-113.

Fisher, M. (1999a). Fetal activity and maternal monitoring methods. British Journal of Midwifery, 7(11), 705-737.

Fisher, M. (1999b). Reduced fetal movements: a research based project. British Journal of Midwifery, 7(12), 733-737.

Flenady, V., Koopmans, L., Middleton, P., Frøen, J. F., Smith, G. C., Gibbons, K., Ezzati, M. (2011). Major risk factors for stillbirth in high-income countries: a systematic review and meta-analysis. The Lancet, 377(9774), 1331-1340. 
Flenady, V., MacPhail, J., Gardener, G., Chadha, Y., Mahomed, K., Heazell, A., Frøen, F. (2009). Detection and management of decreased fetal movements in Australia and New Zealand: A survey of obstetric practice. Australian and New Zealand Journal of Obstetrics and Gynaecology, 49(4), 358-363.

Flenady, V., Middleton, P., Smith, G. C., Duke, W., Erwich, J. J., Khong, T. Y., Frøen, J. F. (2011). Stillbirths: the way forward in high-income countries. The Lancet, 377(9778), 1703-1717.

Froen, J. F. (2004). A kick from within- fetal movement counting and the cancelled progress in antenatal care. Journal of Perinatal Medicine, (32), 13-24.

Frøen, J. F., Cacciatore, J., McClure, E. M., Kuti, O., Jokhio, A. H., Islam, M., \& Shiffman, J. (2011). Stillbirths: why they matter. The Lancet, 377(9774), 13531366.

Froen, J. F., Gardosi, J. O., Thurmann, A., Francis, A., \& Stray-Pedersen, B. (2004). Restricted fetal growth in sudden intrauterine unexplained death. Acta Obstetricia et Gynecologica Scandinavica, 83(9), 801-807.

Frøen, J. F., Heazell, A. E. P., Tveit, J. V. H., Saastad, E., Fretts, R. C., \& Flenady, V. (2008a). Fetal Movement Assessment. Seminars in Perinatology, 32(4), 243-246.

Frøen, J. F., Heazell, A. E. P., Tveit, J. V. H., Saastad, E., Fretts, R. C., \& Flenady, V. (2008b). Fetal Movement Assessment. Seminars in Perinatology, 32(4), 243-246.

Gardosi, J., \& Francis, A. (2009). Adverse pregnancy outcome and association with small for gestational age birthweight by customized and population-based percentiles. American Journal of Obstetrics and Gynecology, 201(1), 28.e1-28.e8. doi:doi: 10.1016/j.ajog.2009.04.034

Gardosi, J., Giddings, S., Clifford, S., Wood, L., \& Francis, A. (2013). Association between reduced stillbirth rates in England and regional uptake of accreditation 
training in customised fetal growth assessment. BMJ Open, 3(12), e003942e003942.

Gettinger, A., Roberts, A., \& Campbell, S. (1978). Comparison between subjective and ultrasound assessments of fetal movement. British Medical Jounal, 2, 88-90.

Giacomini, M. K. (2000). Users' Guides to the Medical Literature: XXIII. Qualitative Research in Health Care B. What Are the Results and How Do They Help Me Care for My Patients? JAMA: The Journal of the American Medical Association, 284(4), $478-482$.

Gillieson, M., Dunlap, H., Nair, R., \& Pilon, M. (1985). Placental site, parity, and date of quickening. Obstetrics and Gynecology, 64(1), 44-45.

Giorgi, A. (1992). Description versus interpretation: competing alternative strategies for qualitative research. Journal of Phenomenological Psychology, 23(2), 119-135.

Goldenberg, R. L., McClure, E. M., Bhutta, Z. A., Belizán, J. M., Reddy, U. M., Rubens, C. E.,Darmstadt, G. L. (2011). Stillbirths: the vision for 2020. The Lancet, 377(9779), 1798-1805.

Goodlin, R. (1979). History of fetal monitoring. American Journal of Obstetrics and Gynecology, 133(3), 323-351.

Goodman, J. D. S. (1980). British Journal of Obstetrics and Gynaecology, 87, 1080-1083.

Grant, A., Elbourne, D., Valentin, L., \& Alexander, S. (1989). Routine formal fetal movement counting and risk of antepartum late death in normally formed singletons. The Lancet, 2, 346-349.

Grbich, C. (2007). Qualitative data analysis: an introduction. London ; Thousand Oaks, Calif: SAGE Publications. 
Groome, L. J., Swiber, M. J., Holland, S. B., Bentz, L. S., \& Atterbury, J. L. (1998). Spontaneous motor activity in the perinatal infant before and after birth: stability in individual differences. Developmental Psychobiology, 35, 15-24.

Habek, D. (2007). Effects of Smoking and Fetal Hypokinesia in Early Pregnancy. Archives of Medical Research, 38(8), 864-867. doi:10.1016/j.arcmed.2007.06.007

Harrington, K., Thompson, O., Jordan, L., Page, J., Carpenter, R., \& Campbell, S. (1998). Obstetric outcome in women who present with a reduction on fetal movements in the third trimester of pregnancy. Journal of Perinatal Medicine, 26, 77-82.

Hay, W. (2006). Placental-fetal glucose exchange and fetal glucose metabolism. Transactions of the American Clinical and Climatological Association, 117, 321340.

Heazell, A. E., Bernatavicius, G., Roberts, S. A., Garrod, A., Whitworth, M. K., Johnstone, E. D., Lavender, T. (2013). A randomised controlled trial comparing standard or intensive management of reduced fetal movements after 36 weeks gestation-a feasibility study. BMC Pregnancy and Childbirth, 13(1), 95.

Heazell, A. E. P., \& Frøen, J. F. (2008). Methods of fetal movement counting and the detection of fetal compromise. Journal of Obstetrics \& Gynaecology, 28(2), 147154.

Heazell, A. E. P., Green, M., Wright, C., Flenady, V., \& Frederik Frøen, J. (2008). Midwives' and obstetricians' knowledge and management of women presenting with decreased fetal movements. Acta Obstetricia et Gynecologica Scandinavica, 87(3), 331-339.

Heazell, A. E. P., Sumathi, G. M., \& Bhatti, N. R. (2005). What investigation is appropriate following maternal perception of reduced fetal movements? Journal of Obstetrics \& Gynaecology, 25(7), 648-650. 
Hertogs, K., Roberts, A., Cooper, D., \& Griffen, D. (1979). Maternal perception of fetal motor activity. British Medical Journal, 2, 1183-1185.

Higgins, L., Greenwood, S. L., Wareing, M., Sibley, C. P., \& Mills, T. A. (2011). Obesity and the placenta: A consideration of nutrient exchange mechanisms in relation to aberrant fetal growth. Placenta, 32(1), 1-7.

Hijazi, Z. R., Callan, S. E., \& East, C. E. (2010). Maternal perception of foetal movement compared with movement detected by real-time ultrasound: An exploratory study. Australian and New Zealand Journal of Obstetrics and Gynaecology, 50(2), 144147.

Hofmeyr, G. J., \& Novikova, N. (2012). Management of reported decreased fetal movements for improving pregnancy outcomes. In The Cochrane Collaboration \& G. J. Hofmeyr (Eds.), Cochrane Database of Systematic Reviews. Chichester, UK: John Wiley \& Sons, Ltd. Retrieved from http://doi.wiley.com/10.1002/14651858.CD009148.pub2

Holden, K. (1984). Increased Fetal Activity with Low Maternal Blood Glucose Levels in Pregnancies Complicated by Diabetes. American Journal of Perinatology, 1(2), $161-164$.

Hsieh, H., \& Shannon, S. (2005). Three Approaches to Qualitative Content Analysis. Qualitative Health Research, 15(9), 1277-1288. doi:10.1177/1049732305276687

Huang, D., Usher, R., Kramer, M., Yang, H., Morin, L., \& Fretts, R. (2000). Determinants of unexplained antepartum fetal death. Obstetrics and Gynecology, 95(2), 215-221.

Hutchinson, S. (2001). The development of qualitative health research: taking stock. Qualitative Health Research, 11(4), 505-521.

Jackson, M., Forouzan, I., \& Cohen, A. (1991). Fetal well-being: nonimaging assessment and the biophysical profile. Seminars in Roentgenology, 36(1), 21-31. 
Jovanovic, L., Kessler, A., \& Peterson, C. (1985). Human maternal and fetal response to graded exercise. Journal of Applied Physiology, 58, 1719-1722.

Kainer, F., Prechtl, H. F., Engele, H., \& Eispeiler, C. (1997). Assessment of the quality of general movements in fetuses of women with type-1 diabetes mellitus. Early Human Development, 50, 13-25.

Kalanithi, L. E. G., Illuzzi, J. L., Nossov, V. B., Frisbæk, Y., Abdel-Razeq, S., Copel, J. A., \& Norwitz, E. R. (2007). Intrauterine Growth Restriction and Placental Location. Journal of Ultrasound in Medicine, 26(11), 1481-1489.

Kintraia, P., Zarnadze, M., Kintraia, N., \& Kashakashvili. (2005). Development of daily rythmicity in heart rate and locomotor activity in the human fetus. Journal of Circadian Rhythms, 3(5), 1-12.

Kisilevsky, B. S., \& Low, J. A. (1998). Human fetal behaviour: 100 years of study. Devevelopmental Review, 18(DR980452), 1-29.

Koenen, S. V., Mulder, E. J. H., Wijnberger, L. D., \& Visser, G. H. A. (2005). Transient Loss of the Diurnal Rhythms of Fetal Movements, Heart Rate, and Its Variation after Maternal Betamethasone Administration. Pediatric Research, 57(5 Part 1), 662-666. doi:10.1203/01.PDR.0000159762.50504.1F

Kumar, A., Devi, S. G., Batra, S., Singh, C., \& Shukla, D. K. (2009). Calcium supplementation for the prevention of pre-eclampsia. International Journal of Gynecology \& Obstetrics, 104(1), 32-36. doi:10.1016/j.ijgo.2008.08.027

Kurjak, A., Talic, A., Honemeyer, U., Stanojevic, M., \& Zalud, I. (2013). Comparison between antenatal neurodevelopmental test and fetal Doppler in the assessment of fetal well being. Journal of Perinatal Medicine, 41(1), 107-114. doi:10.1515/jpm2012-0018 
Kuwata, T., Matsubara, S., Ohkusa, T., Ohkuchi, A., Izumi, A., Watanabe, T., \& Suzuki, M. (2008). Establishing a reference value for the frequency of fetal movements using modified "count to 10" method. Journal of Obstetrics and Gynaecology Research, 34(3), 318-323.

Lalor, J. G., Fawole, B., Alfirevic, Z., \& Devane, D. (2008). Biophysical profile for fetal assessment in high risk pregnancies. In The Cochrane Collaboration \& J. G. Lalor (Eds.), Cochrane Database of Systematic Reviews. Chichester, UK: John Wiley \& Sons, Ltd. Retrieved from http://doi.wiley.com/10.1002/14651858.CD000038.pub2

Lawn, J. E., Blencowe, H., Pattinson, R., Cousens, S., Kumar, R., Ibiebele, I., Stanton, C. (2011). Stillbirths: Where? When? Why? How to make the data count? The Lancet, 377(9775), 1448-1463. doi:10.1016/S0140-6736(10)62187-3

Limesand, S. W., Rozance, P. J., Macko, A. R., Anderson, M. J., Kelly, A. C., \& Hay, W. W. (2012). Reductions in insulin concentrations and beta-cell mass precede growth restriction in sheep fetuses with placental insufficiency. AJP: Endocrinology and Metabolism, 304(5), E516-E523. doi:10.1152/ajpendo.00435.2012

Lincoln, Y., \& Guba, E. (1985). Naturalistic inquiry. Newbury Park, California.: SAGE Publications.

Lindqvist, P. G., \& Molin, J. (2005). Does antenatal identification of small-for-gestational age fetuses significantly improve their outcome? Ultrasound in Obstetrics and Gynecology, 25(3), 258-264.

Liston, R., Bloom, K., \& Zimmer, P. (1994). The psychological effects of counting fetal movements. Birth, 135-140.

Liston, R., Cohen, A., Mennuti, M., \& Gabbe, S. G. (1982). Antepartum fetal evaluation of maternal perception of fetal movements. Obstetrics and Gynecology, (60), 424426. 
Lobb, M., Beazley, J., \& Haddad, N. (1985). A controlled study of daily fetal movement counts in the prevention of stillbirths. Journal of Obstetrics and Gynaecology, (6), 87-91.

Lowery, C., Russell, W., Wilson, J., Walls, R., \& Murphy, P. (1995). Time-quantified fetal movement detection with two-transducer data fusion. American Journal of Obstetrics and Gynecology, 172, 1756-1764.

Luchinger, A., Hadders-Algra, M., Van Kan, C., \& de Vries. (2008). Fetal onset of general movements. Pediatric Research, 63(2), 191-195.

Malm, M.-C., Lindgren, H., \& Rådestad, I. (2010). Losing Contact with One’s Unborn Baby_-Mothers' Experiences Prior to Receiving News that Their Baby has Died in Utero. OMEGA-Journal of Death and Dying, 62(4), 353-367. doi:10.2190/OM.62.4.c

Malterud, K. (2001a). Qualitative research: standards, challenges, and guidelines. The Lancet, 358, 483-358.

Malterud, K. (2001b). The art and science of clinical knowledge: evidence beyond measures and numbers. The Lancet, 358, 397-400.

Manders, M., Sonder, G., Mulder, E., \& Visser, G. (1997). The effects of maternal exercise on fetal heart rate and movement patterns. Early Human Development, 48, $237-247$.

Mangesi, L., Hofmeyr, G. J., \& Smith, V. (2007). Fetal movement counting for assessment of fetal wellbeing. In The Cochrane Collaboration \& L. Mangesi (Eds.), Cochrane Database of Systematic Reviews. Chichester, UK: John Wiley \& Sons, Ltd. Retrieved from http://doi.wiley.com/10.1002/14651858.CD004909.pub2 
Manning, F. A., Platt, L. D., \& Sipos, L. (1980). Antepartum fetal evaluation: development of a fetal biophysical profile. American Journal of Obstetrics and Gynecology, 136(6), 787-795.

Marsal, K. (1983). Ultrasonic assessment of fetal activity. Clinics in Obstetrics and Gynaecology, 10(3), 541-563.

Martin, C. B. (2008). Normal Fetal Physiology and Behavior, and Adaptive Responses with Hypoxemia. Seminars in Perinatology, 32(4), 239-242.

McCowan, L. M. E., George-Haddad, M., Stacey, T., \& Thompson, J. M. D. (2007). Fetal growth restriction and other risk factors for stillbirth in a New Zealand setting. The Australian \& New Zealand Journal of Obstetrics \& Gynaecology, 47(6), 450-456.

Meadows, K. (2003). So you want to do research? 1: an overview of the research process. British Journal of Community Nursing, 8(8), 369-375.

Mikhail, M., Freda, M., Merkatz, R., Polizzotto, R., Mazloom, E., \& Merkatz, I. (1991). The effect of fetal movement counting on maternal attachment to fetus. American Journal of Obstetrics and Gynecology, 165(4), 988-91.

Miller, F., Skiba, H., \& Klapholz, H. (1978). The effect of maternal blood sugar levels on fetal activity. Obstetrics and Gynecology, 52(6), 662-665.

Minors, D. S., \& Waterhouse, J. M. (1979). The effect of maternal, posture, meals and time of day on fetal movements. British Journal of Obstetrics and Gynaecology, $86,717-723$.

Moore, T., \& Piacquadio, K. (1989). A prospective evaluation of fetal movement screening to reduce the incidence of antepartum fetal death. American Journal of Obstetrics and Gynecology, 160, 1075-1080.

Morabia, A. (1996). P.C.A Louis and the birth of clinical epidemiology. Journal of Clinical Epidemiology, 49(12), 1327-1333. 
Morgan, D. (1998). Practical strategies for combining qualitative and quantitative methods: Applications to health research. Qualitative Health Research, 8(3), 362376.

Morokuma, S., Fukushima, K., Yumoto, Y., Uchimura, M., Fujiwara, A., Matsumoto, M., Nakano, H. (2007). Simplified ultrasound screening for fetal brain function based on behavioral pattern. Early Human Development, 83(3), 177-181.

Morse, J. (1991). Approaches to qualitative-quantitative triangulation. Nursing Research, $40,120-123$.

Morse, J. (2011). What is qualitative health research? In The sage handbook of qualitative research (4th ed., pp. 401-413). USA: Sage.

Morse, J., \& Singleton, J. (2001). Exploring the technical aspects of "fit" in qualitative research. Qualitative Health Research, 11(2), 841-847.

Mulder, E., Visser, G. H. A., Bekedam, D. J., \& Prechtl, H. F. . (1987). Emergence of behavioral states in fetuses of type 1 diabetic women. Early Human Development, 15(4), 231-251.

Nabhan, A. F., \& Abdelmoula, Y. A. (2008). Amniotic fluid index versus single deepest vertical pocket as a screening test for preventing adverse pregnancy outcome. Cochrane Database of Systematic Reviews, CD006593.

Neergaard, M., Olesen, F., Andersen, R., \& Sondergaard, J. (2009). Qualitative description - the poor cousin of health research? BMC Medical Research Methodology, 9(1), 52. doi:10.1186/1471-2288-9-52

Neldam, S. (1980). Fetal movements as an indicator of fetal wellbeing. The Lancet, 315(8180), 1222-1224.

Neldam, S., \& Jessen, P. (1980). Fetal movements registered by the pregnant woman correlated to the retrospective estimations of fetal movement from 
cardiotocographic tracings. American Journal of Obstetrics and Gynecology, 136, $1051-1054$.

New Zealand College of Midwives. (2008). Midwives Handbook for Practice (4th ed.). Christchurch [N.Z.]: New Zealand College of Midwives.

New Zealand. Ministry of Health. (2012). Guidelines for consultation with obstetric and related medical services (referral guidelines). Wellington, N.Z.: Ministry of Health. Retrieved from http://www.health.govt.nz/publication/guidelinesconsultation-obstetric-and-related-medical-services-referral-guidelines

NICE. (2008). Antenatal care. Retrieved June 22, 2013, from http://publications.nice.org.uk/quality-standard-for-antenatal-care-qs22

Nicholls, D. (2009). Qualitative research: part one - Philosophies. International Journal of Therapy and Rehabilitation, 16(10), 526-533.

Nijhuis, J. G., Prechtl, H. F., Martin, C. B., Jr, \& Bots, R. S. (1982). Are there behavioural states in the human fetus? Early Human Development, 6(2), 177-195.

Olesen, A. G., \& Svare, J. A. (2004). Decreased fetal movements: background, assessment, and clinical management. Acta Obstetricia et Gynecologica Scandinavica, 83(9), 818-826.

O’Sullivan, O., Stephen, G., Martindale, E., \& Heazell, A. E. P. (2009). Predicting poor perinatal outcome in women who present with decreased fetal movements. Journal of Obstetrics \& Gynaecology, 29(8), 705-710.

Parretti, E., Mecacci, F., Papini, M., Cioni, R., Carignani, L., Mignosa, M., Mello, G. (2001). Third-trimester maternal glucose levels from diurnal profiles in nondiabetic pregnancies. Diabetes Care, 24(8), 1319-1323.

Patrick, J., Campbell, K., Carmicheal, L., Natale, R., \& Richardson, B. (1982). Patterns of gross fetal body movements over 24-hour observation intervals during the last 10 
weeks of pregnancy. American Journal of Obstetrics and Gynecology, 142(4), $363-371$.

Pattison, N., \& McCowan, L. (2010). Cardiotocography for antepartum fetal assessment. In The Cochrane Collaboration \& S. Henderson (Eds.), Cochrane Database of Systematic Reviews. Chichester, UK: John Wiley \& Sons, Ltd. Retrieved from http://doi.wiley.com/10.1002/14651858.CD001068.pub2

Pavlov, I. (1927). Conditioned Reflexes. Oxford University Press.

Pearson, J., \& Weaver, J. (1976). Fetal activity and fetal wellbeing: an evaluation. British Medical Journal, 1, 1305-1307.

Peat, A. M., Stacey, T., Cronin, R., \& McCowan, L. M. E. (2012). Maternal knowledge of fetal movements in late pregnancy. Australian and New Zealand Journal of Obstetrics and Gynaecology, 52(5), 445-449.

Pillai, M., \& James, D. (1990). Behavioural states in normal mature human fetuses. Archives of Disease in Childhood, (65), 39-43.

Pillai, M., James, D., \& Parker, M. (1992). The development of ultradian rhythms in the human fetus. American Journal of Obstetrics and Gynecology, 167, 172-177.

Platt, L. D., Walla, C. A., Paul, R. H., Trujillo, M. E., Loesser, C. V., \& Jacobs, N. D. (1985). A prospective trial of the fetal biophysical profile vs the nonstress test in the management of high-risk pregnancies. American Journal of Obstetrics and Gynecology, 153(6), 624-633.

Polit, D. F. (2008). Nursing research: generating and assessing evidence for nursing practice (8th ed.). Philadelphia: Wolters Kluwer Health/lippincott Williams \& Wilkins. 
Pope, C., \& Mays, N. (1995). Reaching the parts other methods cannot reach: an introduction to qualitative methods in health and health services research. $B M J$ : British Medical Journal, 311(6996), 42-45.

Prechtl, H., \& Einspieler, C. (1997). Is neurological assessment of the fetus possible? European Journal of Obstetrics \& Gynecology and Reproductive Biology, 75, 8184.

Preston, S., Mahomed, K., Flenady, V., Gardener, G., MacPhail, J., Conway, L., ... Froen, F. (2010). Clinical practice guideline for the management of women who report decreased fetal movement. Brisbane: Australia and New Zealand Stillbirth Association (ANZSA).

Pylypa, J. (2009). Elder authority and the situational diagnosis of diarrheal disease as normal infant develpment in Northeast Thailand. Qualitative Health Research, 19(7), 965-975.

Rådestad, I. (2010). Fetal movements in the third trimester - Important information about wellbeing of the fetus. Sexual \& Reproductive Healthcare, 1, 119-121.

Rådestad, I., \& Lindgren, H. (2012). Women's perceptions of fetal movements in full-term pregnancy. Sexual \& Reproductive Healthcare, 3(3), 113-116.

Rayburn, W. (1980). Clinical significance of perceptible fetal motion. American Journal of Obstetrics and Gynecology, 138(2), 210-212.

Rayburn, W., Zuspan, F., Motley, M., \& Donaldson, M. (1980). An alternative to antepartum fetal heart rate testing. American Journal of Obstetrics and Gynecology, 138(2), 223-226.

Raynes-Greenow, C. H., Gordon, A., Li, Q., \& Hyett, J. A. (2013). A cross-sectional study of maternal perception of fetal movements and antenatal advice in a general 
pregnant population, using a qualitative framework. BMC Pregnancy and Childbirth, 13(1), 32. doi:10.1186/1471-2393-13-32

Reece, E. A., Coustan, D. R., \& Gabbe, S. G. (2004). Diabetes in women: adolescence, pregnancy, and menopause. Philadelphia: Lippincott Williams \& Wilkins.

Reinhold, E. (1973). Clinical value of spontaneous movements in early pregnancy. Journal of Perinatal Medicine, 1, 65-69.

Reissland, N., \& Francis, B. (2010). The quality of fetal arm movements as indicators of fetal stress. Early Human Development, 86(12), 813-816.

Robertson, S. S., \& Dierker, L. J. (2003). Fetal cyclic motor activity in diabetic pregnancies: Sensitivity to maternal blood glucose. Developmental Psychobiology, 42(1), 9-16.

Roodenburg, P. J., Wladirimoff, A., van Es, A., \& Prechtl, H. F. (1991). Classification and quantitative aspects of fetal movements during the second half of normal pregnancy. Early Human Development, (25).

Royal Australian and New Zealand College of Obstetricians and Gynecologists. (2012). Timing of elective caesarean section at term (Vol. C-Obs 23).

Saastad, E., Vangen, S., \& Frederik Frøen, J. (2007). Suboptimal care in stillbirths - a retrospective audit study. Acta Obstetricia et Gynecologica Scandinavica, 86(4), $444-450$.

Saastad, E., Winje, B. A., Stray Pedersen, B., \& Frøen, J. F. (2011). Fetal Movement Counting Improved Identification of Fetal Growth Restriction and Perinatal Outcomes - a Multi-Centre, Randomized, Controlled Trial. PLoS ONE, 6(12), e28482. 
Sadovsky, E., Mahler, Y., Polishuk, W., \& Malkin, A. (1973). Correlation between electromagnetic recording and maternal assessment of fetal movement. The Lancet, $1141-1143$.

Sadovsky, E., \& Yaffe, H. (1973). Daily fetal movement recording and fetal prognosis. Obstetrics and Gynecology, 41(6), 845-850.

Sadovsky, E., Yaffe, H., \& Polishuk, W. (1974). Fetal movement monitoring in normal and pathologic pregnancy. International Journal of Gynecology \& Obstetrics, (12), $75-79$.

Salihu, H. M. (2008). Epidemiology of Stillbirth and Fetal Central Nervous System Injury. Seminars in Perinatology, 32(4), 232-238.

Samra, H. A., McGrath, J. M., \& Wehbe, M. (2011). An Integrated Review of Developmental Outcomes and Late-Preterm Birth. Journal of Obstetric, Gynecologic, \& Neonatal Nursing, 40(4), 399-411.

Sandelowski, M. (2000). Whatever Happened to Qualitative Description? Research in Nursing \& Health, 23, 3340340.

Sandelowski, M., \& Barroso, J. (2002). Finding the findings in qualitative studies. Journal of Nursing Scholarship, 34(3), 213-219.

Schmidt, W., Cseh, I., Hara, K., \& Kubli, F. (1984). Maternal perception of fetal movements and real-time ultrasound findings. Journal of Perinatal Medicine, 12, $313-318$.

Schon, D. (2001). The crisis of professional knowledge and the pursuit of an epistemology of practice. In Competence in the learning society (pp. 185-207). New York: Peter Lang.

Silver, R. M. (2011). Previous Stillbirth, Late Preterm, and Early-Term Birth. Seminars in Perinatology, 35(5), 302-308. doi:10.1053/j.semperi.2011.05.006 
Sinha, D., Sharma, A., Nallaswamy, V., Jayagopal, N., \& Bhatti, N. (2007). Obstetric outcome in women complaining of reduced fetal movements. Journal of Obstetrics \& Gynaecology, 27, 41-43.

Sival, D. A., Visser, G. H. A., \& Prechtl, H. F. (1990). Does reduction in amniotic fluid affect fetal movements? Early Human Development, (23), 233-247.

Sival, D. A., Visser, G. H. A., \& Prechtl, H. F. (1992a). The effect of intrauterine growth retardation on the quality of general movements in the human fetus. Early Human Development, 28, 119-132.

Sival, D. A., Visser, G. H. A., \& Prechtl, H. F. (1992b). The relationship between quantity and quality of prenatal movements in pregnancies complicated by intra-uterine growth retardation and premature rupture of the membranes. Early Human Development, 30, 193-209.

Skornick-Rapaport, A., Maslovitz, S., Kupferminc, M., Lessing, J. B., \& Many, A. (2011). Proposed management for reduced fetal movements: five years' experience in one medical center. Journal of Maternal-Fetal and Neonatal Medicine, 24(4), 610-613.

Smith, G. C. (2011). A bonfire of the tape measures. The Lancet, 377(9774), 1307.

Smith, V., Begley, C., \& Devane, D. (2014). Detection and management of decreased fetal movements in Ireland: A national survey of midwives' and obstetricians' practices. Midwifery, 30(1), 43-49.

Stacey, T., Thompson, J. M. D., Mitchell, E. A., Ekeroma, A. J., Zuccollo, J. M., \& McCowan, L. M. E. (2011). Association between maternal sleep practices and risk of late stillbirth: a case-control study. BMJ, 342(jun14 1), d3403-d3403.

Stacey, T., Thompson, J. M. D., Mitchell, E., Ekeroma, A., Zuccollo, J., \& McCowan, L. (2011a). Maternal perception of fetal activity and late stillbirth risk; findings from the Auckland stillbirth study. Birth, 38(4), 1-6. 
Stacey, T., Thompson, J. M. D., Mitchell, E., Ekeroma, A., Zuccollo, J., \& McCowan, L. M. E. (2011b). Relationship between obesity, ethnicity and risk pf late stillbirth: a case control study. BMC Pregnancy and Childbirth, 11(3).

Stark, R., Garland, M., Daniel, S., Tropper, P., \& Myers, M. (1999). Diurnal rhythms of fetal and maternal heart rate in the baboon. Early Human Development, 55, 195209.

Strubbe, J., \& Dijk, G. (2002). The temporal organisation of ingestive behaviour and its interaction with regulation of energy balance. Neuroscience and Biobehavioral Reviews, 26, 485-498.

Teddlie, C., \& Tashakkori, A. (2006). A general typology of research designs featuring mixed methods. Research in the Schools, 13(1), 12-28.

Thorne, S., Kirkham, S., \& MacDonald-Emes, J. (1997). Interpretive description: a noncategorical qualitative alternative for developing nursing knowledge. Research in Nursing \& Health, 20, 169-177.

Thorne, S., Reimer Kirkham, S., \& O'Flynn-Magee, K. (2004). The analytic challenge in interpretive description. International Journal of Qualitative Methods, 3(1), 1-21.

Tuffnell, D., Cartmill, R., \& Lilford, R. (1991). Fetal Movements; factors affecting their perception. European Journal of Obstetrics \& Gynecology and Reproductive Biology, 39(3), 165-167.

Tveit, J., Saastad, E., Stray-Pedersen, B., Børdahl, P. E., Flenady, V., Fretts, R., \& Frøen, J. F. (2009). Reduction of late stillbirth with the introduction of fetal movement information and guidelines - a clinical quality improvement. BMC Pregnancy and Childbirth, 9(1), 32. doi:10.1186/1471-2393-9-32

Tveit, J. V. H., Saastad, E., Stray-Pedersen, B., Børdahl, P. E., \& Frøen, J. F. (2010). Concerns for decreased foetal movements in uncomplicated pregnancies - 
Increased risk of foetal growth restriction and stillbirth among women being overweight, advanced age or smoking. Journal of Maternal-Fetal and Neonatal Medicine, 23(10), 1129-1135. doi:10.3109/14767050903511578

Tveit, J. V., Saastad, E., Stray-Pedersen, B., Børdahl, P. E., \& Frøen, J. F. (2009). Maternal characteristics and pregnancy outcomes in women presenting with decreased fetal movements in late pregnancy. Acta Obstetricia et Gynecologica Scandinavica, 88(12), 1345-1351.

Unterscheider, J., Horgan, R. P., Greene, R. A., \& Higgins, J. R. (2010). The management of reduced fetal movements in an uncomplicated pregnancy at term: Results from an anonymous national online survey in the Republic of Ireland. Journal of Obstetrics \& Gynaecology, 30(6), 578-582.

Unterscheider, J., \& O’Donoghue, K. (2012). Reduced fetal movements. In From preconception to postpartum, Dr. Stavros Sifakis (Ed.) (p. 314). InTech.

Van Leeuwen, P., Geue, D., Lange, S., Cysarz, D., Bettermann, H., \& Gronemeyer, D. (2003). Is there evidence of fetal-maternal heart rate synchronization? BMC Physiology, 3(2), 1-11.

Van Leeuwen, P., Geue, D., Lange, S., \& Gronemeyer, D. (2009). Modeling fetal-maternal heart-rate interaction. IEEE Engineering in Medicine and Biology Magazine, 28(6), $49-53$.

Van Leeuwen, P., Geue, D., Thiel, M., Cysarz, D., Lange, S., Romano, M. C., Gronemeyer, D. H. (2009). Influence of paced maternal breathing on fetal-maternal heart rate coordination. Proceedings of the National Academy of Sciences, 106(33), $13661-13666$.

Van Vliet, M. A., Martin, C. B., Jr, Nijhuis, J. G., \& Prechtl, H. F. (1985). The relationship between fetal activity and behavioral states and fetal breathing movements in 
normal and growth-retarded fetuses. American Journal of Obstetrics and Gynecology, 153(5), 582-588.

Visser, G. H. A., Mulder, E. J. H., \& Tessa Ververs, F. F. (2010). Fetal behavioral teratology. Journal of Maternal-Fetal and Neonatal Medicine, 23(S3), 14-16.

Warner, J., Hains, S. M. J., \& Kisilevsky, B. S. (2002). An exploratory study of fetal behavior at 33 and 36 weeks gestational age in hypertensive women. Developmental Psychobiology, 41(2), 156-168. doi:10.1002/dev.10062

Warrander, L. K., Batra, G., Bernatavicius, G., Greenwood, S. L., Dutton, P., Jones, R. L., Heazell, A. E. P. (2012). Maternal Perception of Reduced Fetal Movements Is Associated with Altered Placental Structure and Function. PLoS ONE, 7(4), e34851. doi:10.1371/journal.pone.0034851

Warrander, L. K., \& Heazell, A. E. . (2011). Identifying placental dysfunction in women with reduced fetal movements can be used to predict patients at increased risk of pregnancy complications. Medical Hypotheses, 76(1), 17-20.

Watts, A. G. (2000). Understanding the Neural Control of Ingestive Behaviors: Helping to Separate Cause from Effect with Dehydration-Associated Anorexia. Hormones and Behavior, 37(4), 261-283.

Westgate, J., \& Jamieson, M. (1986). Stillbirths and fetal movements. New Zealand Medical Journal, 99(796), 114-116.

Whitaker, R., \& Dietz, W. (1998). Role of prenatal environment in the development of obesity. Journal of Pediatrics, 132, 768-767.

White, C., Simon, M., \& Bryan, A. (2002). Using evidence to educate birthing center nursing staff about infant states, cues and behaviors. MCN, 27(5), 294-298.

Whittemore, R., Chase, S., \& Mandle, C. (2001). Validity in Qualitative Health Research. Qualitative Health Research, 11(4), 522-537. 
Whitworth, M., Fisher, M., \& Heazell, A. (2011). Reduced Fetal Movements. In Green-top Guideline (Vol. 57, p. 16). Royal College of Obstetricians and Gynecologists.

Wilcox, A. (2001). On the importance -and unimportance- of birthweight. International Journal of Epidemiology, 30, 1233-1241.

Winje, B. A., Røislien, J., \& Frøen, J. F. (2012). Temporal patterns in count-to-ten fetal movement charts and their associations with pregnancy characteristics: a prospective cohort study. BMC Pregnancy and Childbirth, 12, 124.

Winje, B. A., Roald, B., Kristensen, N. P., \& Frøen, J. F. (2012). Placental Pathology in Pregnancies with Maternally Perceived Decreased Fetal Movement - A PopulationBased Nested Case-Cohort Study. PLoS ONE, 7(6), e39259.

Winje, B., Saastad, E., Gunnes, N., Tveit, J., Stray-Pedersen, B., Flenady, V., \& Frøen, J. (2011). Analysis of "count-to-ten” fetal movement charts: a prospective cohort study. BJOG: An International Journal of Obstetrics \& Gynaecology, 118, 12291238.

Yee, W., Amin, H., \& Wood, S. (2008). Elective caesarean delivery, neonatal intensive care unit admission, and neonatal respiratory distress. American Journal of Obstetrics and Gynecology, 11, 823-828.

Zimmer, E., Peretz, B., Marcovici, R., Goldstein, I., Eyal, A., \& Paldi, E. (1982).

Correlation of fetal movements with maternal posture. Journal of Obstetrics and Gynaecology, 3, 85-86.

Zisser, H. (2006). The Fidgety Fetus Hypothesis: Fetal activity is an additional variable in determining birth weight of offspring of women with diabetes. Diabetes Care, 29(1), 63-67. 


\section{Appendix 1 Interview Prompts}

\section{Maternal Perception of Fetal Movements: a qualitative description (first interview)}

\section{Interview Questions/prompts}

Tell me when you first became aware of the baby's movements? (how many weeks, what alerted you to the sensation?)

How would you describe these first baby movements to someone who has never been pregnant?

How would you describe your baby's movements now? (normally/most days)

Does your baby's have a recognisable pattern to their movements?

Can you describe it?

Does your baby move more or less at certain times of day? Yes/No

-If so when do they move more? When do they move less?

Does your baby move more or less in response to different positions or activities? Yes/No If so which positions? Which activities?

Do you actively/consciously think much about the baby's movements during the day?

Are you aware of baby movements at night? Yes/No

If so, describe this?

How many times would you say your baby has moved today?

(Distinct movements? Active spells?)

How many times would you say your baby moved in the last hour?

How often would you say your baby moves in a normal day?

Does your midwife (or doctor) ask you about baby movements? Yes/No

-If so, what do they ask you?

Has your midwife (or doctor) given you information about baby movements? Yes/No -What did they tell you

Have your family or friends told you anything about baby movements?

-If so, what did they tell you?

Have you ever felt concerned about your baby's moments? Yes/No

-What prompted your concern? 


\section{Maternal Perception of Fetal Movements: a qualitative description TERM}

\section{Introduction}

Thank you for agreeing to talk to me today. As you know I am interested to hear how you have been finding the movements recently.

Interview Questions/prompts

How would you describe the movements you have been having now you are due?

Are the movements the same or different now compared to when we spoke earlier?

How would you describe your baby's movements now? (normally/most days)

Does your baby's have a recognisable pattern of movements?

Can you describe it?

Does your baby move more or less at certain times of day? Yes/No

-If so when do they move more? When do they move less?

Does your baby move more or less in response to different positions or activities? Yes/No If so which positions? Which activities?

Do you actively/consciously think much about the baby's movements during the day?

Are you aware of baby movements at night? Yes/No

If so, describe this?

How many times would you say your baby has moved today?

(Distinct movements? Active spells?)

How many times would you say your baby moved in the last hour?

How often would you say your baby moves in a normal day?

Does your mid wife (or doctor) ask you about baby movements? Yes/No

-If so, what do they ask you?

Has your midwife (or doctor) given you information about baby movements? Yes/No -What did they tell you

Have your family or friends told you anything about baby movements?

-If so, what did they tell you?

Have you ever felt concerned about your baby's moments? Yes/No

-What prompted your concern? 


\section{Appendix 2 Information Sheet}

Maternal Perception of Fetal Movements; a qualitative description

Thank you for agreeing to take part in the Baby Movement study. Midwives and doctors know that an active baby is usually a healthy one and women too find it reassuring when their baby is active in the womb. Midwives and doctors will generally do extra monitoring if a woman reports her baby's movements have lessened. Yet, little is known about how women normally describe baby movements or what exactly is a normal amount of baby movement for a woman to report.

To improve our understanding of how women perceive and describe their baby's movements in the womb, I am conducting a short series of interviews with women in the latter part of pregnancy. Participation involves;

a) A short interview (15 to 30 minutes) asking you about your baby's movements during pregnancy. The interview will be taped and transcribed and notes will be taken recording your comments. Your pregnancy, labour and birth record from the hospital will also be accessed (with your permission) to look for key outcomes that might be relevant in the context of baby's movements during pregnancy such as sex of baby, size of baby, position of your placenta during pregnancy).

b) A second interview will be conducted after 37 weeks of pregnancy. This can be by telephone or in person (as you prefer) and shouldn't take any longer than 10 minutes.

The information gained from the interviews will be considered alongside that of other participants, so that a 'picture' can be built up of the variation in the language women use to describe their baby's movement patterns. A better understanding of how women describe their baby's movements may help to improve baby movement monitoring methods and pregnancy care in the future.

Note; Any information about you will be confidential and only seen by the researcher or research supervisor. No information about you will be passed on to your LMC or to the hospital and no information identifying you personally will be published in the final report. 


\section{Appendix 3 Consent Form}

Maternal Perception of Fetal Movements: a qualitative description

- I have read and understand the information sheet dated. I have had the opportunity to discuss this study with Billie Clayton and I am happy with the answers I have been given.

- I understand that taking part in this study is my choice and that I can withdraw from the study at any time, and this will in no way affect my pregnancy care.

- I agree to be contacted by telephone for a second follow-up interview after 37 weeks of pregnancy and that each interview will take up no more than 15 to 30 minutes of my time.

- I understand my pregnancy, labour and birth record will be accessed for the purposes of this study and the information within will be confidential to the researcher and the research supervisor.

- I understand the findings of the report will be published in professional journals, will be presented at conferences and a copy of the completed thesis will be lodged in the Victoria University of Wellington Library and at the Graduate School of Nursing, Midwifery and Health.

- I understand that no information identifying me will be published in the final report. I also understand that the researcher may use the services of a transcriber who will be bound by a confidentiality agreement.

- Should issues arise I am at liberty to contact the researcher Billie Clayton, her supervisors Robyn Maude, Victoria University of Wellington; Ph (04) 4636167; email Robyn.Maude@vuw.ac.nz or Dr Joan Skinner, Victoria University of Wellington; Ph: (04) 4636654; e-mail: Joan.Skinner@vuw.ac.nz

I agree to participate in this study under the conditions set out above, and I agree to participate in the two interviews.

Name of participant:

Signature of Participant: 


\section{Appendix 4 Coding Sample}

Interview excerpt, 30 weeks.

“A; Now they are quite, I suppose you could say 'vigorous'. They can be quite strong. Umm, umm, sometimes, like lately, they can be kind of so strong that they are almost a little bit, well like they are painful. But...

Q; Does it bother you?

A; Um no well its kind of reassuring, I suppose. At first it did the other day, it kind of took me by surprise. It was like oh woah what was that?! But um yeah, I suppose it's good because you can feel the baby moving. But yeah now it is definitely more, like there is rolling movements and pressing movements."

\section{Codes}

Movements are vigorous, so strong they are almost painful

It is reassuring to feel strong movements

'Woah, what was that?!' Some movements surprisingly strong

More rolling movements and pressing movements (variation)

\section{Category}

Movements are strong, but varied in strength and type

\section{Theme}

It is reassuring to feel the baby's strong movements 